WSRC-TR-99-00090, Rev. 0

\title{
Ingestion Pathway Consequences of a Major Release from SRTC
}

by

A. Blanchard

Westinghouse Savannah River Company

Savannah River Site

Aiken, South Carolina 29808

J. Thompson

Westinghouse Safety Management Solutions

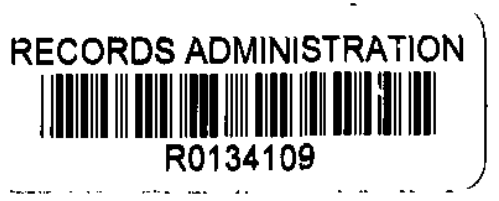


WSRC-TR-99-00090

Revision 0

\section{Ingestion Pathway Consequences of a Major Release from SRTC (U)}

Jay M. Thompson

April 1999

Westinghouse Savannah River Company

Technical Services Division

Aiken, SC 29808

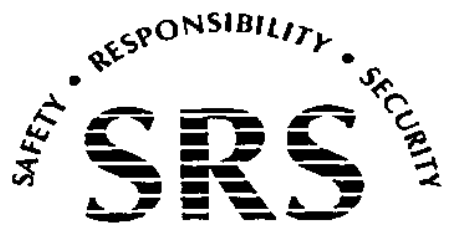

SAVANNAH RIVER SITE

PREPARED FOR THE U.S. DEPARTMENT OF ENERGY UNDER CONTRACT NO. DE-AC09-96SR18500 


\section{DISCLAIMER}

This report was prepared as an account of work sponsored by an agency of the United States Government. Neither the United States Government nor any agency thereof, nor any of their employees, makes any warranty, express or implied, or assumes any legal liability or responsibility for the accuracy, completeness, or usefulness of any information, apparatus, product, or process disclosed, or represents that its use would not infringe privately owned rights. Reference herein to any specific commercial product, process, or service by trade name, trademark, manufacturer, or otherwise does not necessarily constitute or imply its endorsement, recommendation, or favoring by the United States Government or any agency thereof. The views and opinions of authors expressed herein do not necessarily state or reflect those of the United States Government or any agency thereof.

This report has been reproduced directly from the best available copy.

Available to DOE and DOE contractors from the Office of Scientific and Technical Information, P.O. Box 62, Oak Ridge, TN 37831; prices available from (615) 576-8401.

Available to the public from the National Technical Information Service, U.S. Department of Commerce, 5285 Port Royal Road, Springfield, VA 22161. 
WSRC-TR-99-00090

Revision 0

\begin{abstract}
The food ingestion consequences due to radioactive particulates of an accidental release, scenario 1-RD-3, are evaluated for SRTC. The sizes of land areas requiring the

protective action of food interdiction are calculated. The consequences of the particulate portion of the release are evaluated with the HOTSPOT model and an EXCEL spreadsheet for particulates.
\end{abstract}




\section{CONTENTS}

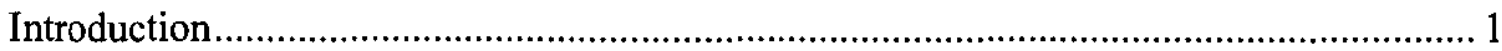

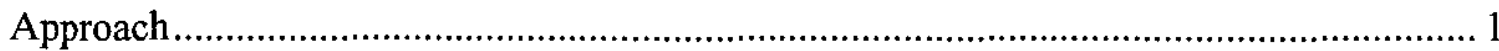

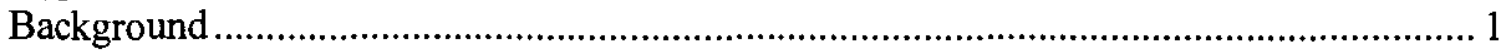

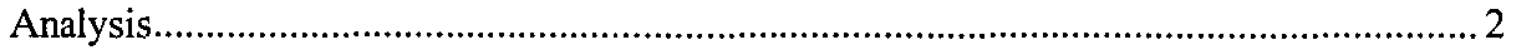

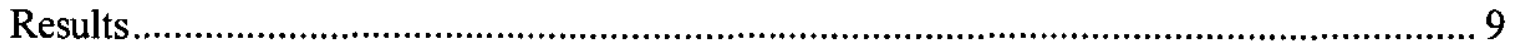

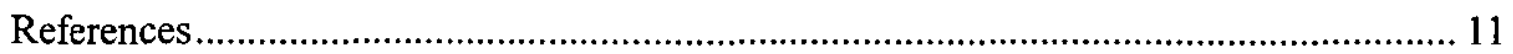

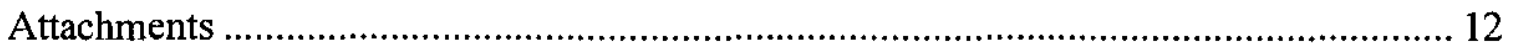

\section{TABLES}

Table 1. Airborne releases for SRTC scenario 1-RD-3, with mixes and DR not applied .. 3

Table 2. Pu 239/241 mix activity fractions ............................................................... 3

Table 3. Pu 242 mix activity fractions .................................................................. 4

Table 4. Total airborne releases for SRTC scenario 1-RD-3, unmixed and with DR

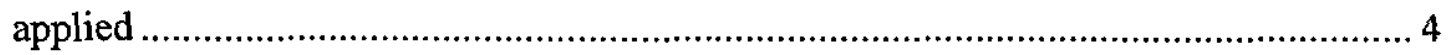

Table 5. Areal concentrations of particulates per unit deposition of the mix .................... 5

Table 6. Radionuclide Group concentrations in milk per unit deposition of the mix........ 6

Table 7. Derived Response Levels, dairy pathway ............................................................ 6

Table 8. Derived Response Levels for ingestion pathways .............................................. 7

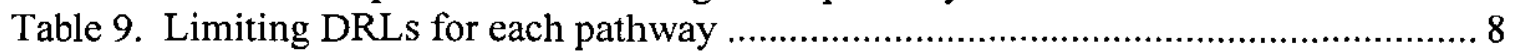

Table 10. HOTSPOT input parameters for SRTC releases ............................................. 8

Table 11. Ranges until deposition less than DRL, average meteorology, SRTC .............. 9

Table 12. Areas above DRL, average meteorology, SRTC .............................................. 9

Table 13. Ranges until deposition less than DRL, 95\% adverse meteorology, SRTC .... 10

Table 14. Areas above DRL, 95\% adverse meteorology, SRTC.................................... 10 


\section{Ingestion Pathway Consequences of a Major Release from SRTC (U)}

\section{Introduction}

Accidental releases of radionuclides from nuclear facilities may cause contamination of food and forage. Numerous pathways exist for deposited radionuclides to reach man. The major pathways considered by recent Food and Drug Administration guidance are analyzed in this report.

\section{Approach}

The methodology for determining the limiting radionuclide group, and Derived Response Level (DRL), for ingestion pathways is outlined below.

1. Determine the source terms (radionuclide-specific and total airborne releases).

2. If tritium is present in the source term, evaluate the consequences of the tritium release using UFOTRI. These results will be summarized in a separate report.

3. Calculate the concentrations of each radionuclide in foodstuffs for a unit deposition of the particulate mix.

4. For radionuclides with Food and Drug Administration (FDA) Derived Intervention Levels (DILs) (FDA 1998), divide the DIL by the foodstuff concentration from a unit deposition. The Derived Response Level, or DRL, is the deposition that gives a DIL for a specific nuclide (group) and pathway.

5. Run the dispersion/deposition model HOTSPOT (Homann 1994) to determine areas impacted.

\section{Background}

The FDA (1998) places selected radionuclides into five groups. These groups are:

- $\operatorname{Sr}-90$

- I-131

- $\mathrm{Cs}-134+\mathrm{Cs}-137$

- $\mathrm{Pu}-238+\mathrm{Pu}-239+\mathrm{Am}-241$

- $\mathrm{Ru}-103+\mathrm{Ru}-106$

Notes for Table 2 and Appendix D of FDA (1998) provide guidance on how to utilize these groups. The Derived Intervention Level (DIL) for each radionuclide group is applied independently (i.e., there is no additivity between groups). If multiple radionuclides are present in a group, the DIL applies to the sum of the concentrations of 
those radionuclides. The DILs are applied to the wet weight of foods (as prepared for consumption).

These groups were developed to aid analyses at a variety of nuclear facilities. Nuclear waste storage facilities and nuclear fuel reprocessing facilities are expected to have $\mathrm{Sr}-90$, Cs-137, Pu-239, and Pu-238 as major contributors (p. 51 of FDA 1998).

FDA recommends that DILs from the five groups be applied immediately following an accident. Early evaluation of other radionuclides that may have been released is not required. However, an evaluation should be performed as soon as possible to ensure the DILs are appropriate; presumably, this would include evaluating DILs for the five groups as well as those for radionuclides not in the groups.

The five radionuclide groups are not sufficient to cover accidents at all types of facilities or locations. FDA (1998) notes the example of transportation accidents that may release radionuclides not in the radionuclide groups. Although such releases are not specifically addressed (in the five groups), FDA recommends that an evaluation of the radiation dose from ingestion of these other radionuclides be performed to determine if protective action guides will be exceeded. Appendix E of FDA (1998) presents DILs for 15 additional radionuclides.

Some source terms at SRS are postulated to be dominated by tritiated water vapor. DILs for tritium in water vapor and organically bound forms were derived in WSRC-TR-9900064 using methodology consistent with FDA (1998). The tritium-specific model UFOTRI will be used to evaluate tritium releases, and the results presented in a separate report (see WSRC-TR-99-00117).

As noted in WSRC-TR-98-0392, the new FDA guidelines appear to be much more restrictive than the old guidelines. This results in greater ranges and larger areas impacted for a given release.

\section{Analysis}

\section{Derivation of the Total Release}

The respirable source terms for a hypothetical SRTC release scenario 1-RD-3 are found in Attachments 2, 3, and 5 of S-EHA-A-00001, Rev. 1, Appendix B, Calculation 1. These respirable source terms are derived from a detailed analysis involving consideration of physical forms and dispersing mechanisms. An overall damage ratio (DR) is applied to the overall source term from the Attachments in S-EHA-A-00001, Rev. 1, Appendix B, Calculation 1 (see sheet 8 of 30 for the DR specification). To determine the total release, it is necessary to "back out" the respirable fraction (RF) from the respirable release source terms. This is done in calculation S-CLC-A-00104 and summarized in Table 1. The activities in Table 1 do not include a correction for a DR less than unity, and the Pu-239/241 and Pu-242 activities are for mixes. 
Table 1. Airborne releases for SRTC scenario 1-RD-3, with mixes and DR not applied

\begin{tabular}{cr}
\hline Nuclide & Pre-DR airborne release (Ci) \\
\hline Am241 & $1.21 \mathrm{E}-02$ \\
Am243 & $9.68 \mathrm{E}-04$ \\
Ba133 & $5.38 \mathrm{E}-13$ \\
Ce144 & $1.90 \mathrm{E}-05$ \\
Cf249 & $1.27 \mathrm{E}-05$ \\
Cf252 & $1.59 \mathrm{E}-02$ \\
Cm244 & $2.03 \mathrm{E}-02$ \\
Cm246 & $1.00 \mathrm{E}-03$ \\
Co60 & $7.19 \mathrm{E}-01$ \\
Cs137 & $1.19 \mathrm{E}+00$ \\
H3 & $2.14 \mathrm{E}+04$ \\
Np237 & $4.20 \mathrm{E}-05$ \\
Pm147 & $2.52 \mathrm{E}-08$ \\
Pu238 & $5.10 \mathrm{E}-02$ \\
Pu239 & $1.78 \mathrm{E}-05$ \\
Pu239/241mix & $4.56 \mathrm{E}-01$ \\
Pu240 & $9.72 \mathrm{E}-07$ \\
Pu241 & $2.91 \mathrm{E}-01$ \\
Pu242mix & $1.28 \mathrm{E}-02$ \\
Sr90 & $1.18 \mathrm{E}+00$ \\
Tc99 & $3.60 \mathrm{E}-13$ \\
Th232 & $1.93 \mathrm{E}-06$ \\
T1204 & $9.00 \mathrm{E}-10$ \\
U235 & $1.78 \mathrm{E}-06$ \\
U238 & $2.15 \mathrm{E}-04$ \\
\hline & 5
\end{tabular}

Reference: SourceSRTC.xls, worksheet "Nonresp + resp", column F

Two additional steps are applied to the potential release activities in Table 1. First, the $\mathrm{Pu}-239 / 241$ and $\mathrm{Pu}-242$ mixes must be separated into their constituents. The makeups of these mixes are in Tables 2 and 3.

Table 2. Pu 239/241 mix activity fractions

\begin{tabular}{cc}
\hline nuclide & activity fraction \\
\hline Pu 238 & 0.0073 \\
Pu239 & 0.2029 \\
Pu240 & 0.0462 \\
Pu241 & 0.6761 \\
Pu242 & 0.0003 \\
Am241 & 0.0675 \\
\hline Reference: S-CLC-A-00080 Rev. 0, Sheet 4 of 26
\end{tabular}


Table 3. Pu 242 mix activity fractions

\begin{tabular}{lc}
\hline nuclide & activity fraction \\
\hline Pu 238 & 0.0786 \\
Pu239 & 0.00024 \\
Pu240 & 0.01184 \\
Pu241 & 0.88886 \\
Pu242 & 0.00074 \\
Am241 & 0.01977 \\
\hline
\end{tabular}

Reference: S-CLC-A-00080 Rev. 0, Sheet 4 of 26

The final step is to apply a damage ratio (DR) of 0.75 , consistent with the DR assumed for respirable releases in the SRTC EPHA. These total releases are shown in Table 4.

Table 4. Total airborne releases for SRTC scenario 1-RD-3, unmixed and with DR applied

\begin{tabular}{cc}
\hline Nuclide & DR=0.75, unmixed total release (Ci) \\
\hline Am241 & $3.23 \mathrm{E}-02$ \\
Am243 & $7.26 \mathrm{E}-04$ \\
Ba133 & $4.04 \mathrm{E}-13$ \\
Ce144 & $1.42 \mathrm{E}-05$ \\
Cf249 & $9.53 \mathrm{E}-06$ \\
Cf252 & $1.19 \mathrm{E}-02$ \\
Cm244 & $1.53 \mathrm{E}-02$ \\
Cm246 & $7.50 \mathrm{E}-04$ \\
Co60 & $5.40 \mathrm{E}-01$ \\
Cs137 & $8.91 \mathrm{E}-01$ \\
$\mathrm{H} 3$ & $1.61 \mathrm{E}+04$ \\
Np237 & $3.15 \mathrm{E}-05$ \\
Pm147 & $1.89 \mathrm{E}-08$ \\
Pu238 & $4.15 \mathrm{E}-02$ \\
Pu239 & $6.94 \mathrm{E}-02$ \\
Pu240 & $1.59 \mathrm{E}-02$ \\
Pu241 & $4.58 \mathrm{E}-01$ \\
Pu242 & $1.10 \mathrm{E}-04$ \\
Sr90 & $8.84 \mathrm{E}-01$ \\
Tc99 & $2.70 \mathrm{E}-13$ \\
Th232 & $1.45 \mathrm{E}-06$ \\
T1204 & $6.75 \mathrm{E}-10$ \\
U235 & $1.34 \mathrm{E}-06$ \\
U238 & $1.62 \mathrm{E}-04$ \\
\hline
\end{tabular}

Reference: SourceSRTC.xIs, Worksheet "Nonresp + resp", columns L to N Details of these spreadsheet calculations are presented in Attachment A. 
Because tritium behaves differently in the environment than particulates, tritium and particulates are analyzed as two separate groups using two different models. The analysis of the tritium release will be described in a separate report.

\section{Evaluation of the deposition needed to exceed a DIL}

To determine the deposition that results in a DIL of a particular radionuclide group in a given foodstuff for a particular pathway, the concentration of radionuclides in foodstuffs is calculated for a unit deposition of particulates $\left(1 \mathrm{Ci} \mathrm{m}^{-2}\right)$. Dividing the DIL by the concentration per unit deposition gives the deposition that results in a DIL.

Assuming that all radionuclides deposit similarly, the relative concentrations of the deposited particulate material will be the same as that released. The areal concentrations of the individual radionuclides present for this unit deposition are given in Table 5.

Table 5. Areal concentrations of particulates per unit deposition of the mix

\begin{tabular}{cc}
\hline Nuclide & $\begin{array}{c}\text { Areal concentration per unit } \\
\text { deposition of particulates } \\
\left(\text { (i m }^{-2}\right)_{\text {nuclide }} \text { per }\left(\mathrm{Ci} \mathrm{m}^{-2}\right)_{\text {mix }}\end{array}$ \\
\hline Am241 & $1.09 \mathrm{E}-02$ \\
Am243 & $2.45 \mathrm{E}-04$ \\
Ba133 & $1.36 \mathrm{E}-13$ \\
Ce144 & $4.80 \mathrm{E}-06$ \\
Cf249 & $3.22 \mathrm{E}-06$ \\
Cf252 & $4.03 \mathrm{E}-03$ \\
Cm244 & $5.15 \mathrm{E}-03$ \\
Cm246 & $2.53 \mathrm{E}-04$ \\
Co60 & $1.82 \mathrm{E}-01$ \\
Cs137 & $3.01 \mathrm{E}-01$ \\
Np237 & $1.06 \mathrm{E}-05$ \\
Pm147 & $6.38 \mathrm{E}-09$ \\
Pu238 & $1.40 \mathrm{E}-02$ \\
Pu239 & $2.34 \mathrm{E}-02$ \\
Pu240 & $5.37 \mathrm{E}-03$ \\
Pu241 & $1.55 \mathrm{E}-01$ \\
Pu242 & $3.70 \mathrm{E}-05$ \\
Sr90 & $2.99 \mathrm{E}-01$ \\
Tc99 & $9.12 \mathrm{E}-14$ \\
Th232 & $4.90 \mathrm{E}-07$ \\
T2204 & $2.28 \mathrm{E}-10$ \\
U235 & $4.52 \mathrm{E}-07$ \\
U238 & $5.45 \mathrm{E}-05$ \\
\hline
\end{tabular}

Reference: SourceSRTC.xls, Worksheet "Nonresp + resp", column R Details of these spreadsheet calculations are presented in Attachment A. 
Three of the five FDA radionuclide groups have one or more members present in the SRTC release scenario. These groups are Sr-90, Cs, and the Pu-238 + Pu-239+ Am-241 group. In addition, 4 of the additional 15 radionuclides with DILs determined in FDA (1998) are present (Ce-144, Cm-244, Np-237, and Pu-241). Evaluation of radionuclide concentrations in foods in the following analysis is limited to radionuclides with FDA DILs, although the total release of all particulate radionuclides is used to describe the unit release.

The concentrations in various foodstuffs due to this unit deposition may now be evaluated. The concentration of a particular radionuclide in a foodstuff is equal to the radionuclide-specific deposition times the (radionuclide-specific) overall transfer factor. Division of the DIL by the concentration in the foodstuff resulting from a unit deposition gives the deposition needed for a DIL. This process is presented in Tables 6 and 7 for the dairy pathway. Details of the spreadsheet calculations are presented in Attachment B.

Transfer factors for plutonium and americium are derived in WSRC-TR-99-00005, Ingestion Pathway Transfer Factors for Plutonium and Americium. Transfer factors for additional radionuclides are presented in S-CLC-G-00179.

Table 6. Radionuclide Group concentrations in milk per unit deposition of the mix

\begin{tabular}{ccccc}
\hline Nuclide & $\begin{array}{c}\text { Areal concentration } \\
\text { per unit deposition } \\
\text { of } \\
\text { particulates } \\
\mathrm{Ci} / \mathrm{m}^{2}\end{array}$ & $\begin{array}{c}\text { Overall transfer } \\
\text { factor } \mathrm{T}_{\text {milk }} \\
\left(\mathrm{m}^{2} \mathrm{~L}^{-1}\right)\end{array}$ & $\begin{array}{c}\text { Radionuclide Group } \\
\text { concentration } \\
\left(\mathrm{Ci} \mathrm{L}^{-1}\right) \text { per unit } \\
\text { deposition of mix }\end{array}$ & $\begin{array}{c}\text { Radionuclide Group } \\
\text { or individual radionuclide }\end{array}$ \\
\hline Am241 & $1.09 \mathrm{E}-02$ & $1.21 \mathrm{E}-05$ & $4.66 \mathrm{E}-07$ & Pu-238+ Pu-239+ Am-241 \\
Pu238 & $1.40 \mathrm{E}-02$ & $8.90 \mathrm{E}-06$ & & \\
Pu239 & $2.34 \mathrm{E}-02$ & $8.90 \mathrm{E}-06$ & & $\mathrm{Ce} 144$ \\
$\mathrm{Ce} 144$ & $4.80 \mathrm{E}-06$ & $2.43 \mathrm{E}-04$ & $1.17 \mathrm{E}-09$ & $\mathrm{Cm} 244$ \\
$\mathrm{Cm} 244$ & $5.15 \mathrm{E}-03$ & $1.62 \mathrm{E}-05$ & $8.33 \mathrm{E}-08$ & $\mathrm{Cs} 137$ \\
$\mathrm{Cs} 137$ & $3.01 \mathrm{E}-01$ & $6.39 \mathrm{E}-02$ & $1.92 \mathrm{E}-02$ & $\mathrm{~Np} 237$ \\
$\mathrm{~Np} 237$ & $1.06 \mathrm{E}-05$ & $4.04 \mathrm{E}-05$ & $4.30 \mathrm{E}-10$ & $\mathrm{Pu} 241$ \\
$\mathrm{Pu} 241$ & $1.55 \mathrm{E}-01$ & $8.90 \mathrm{E}-06$ & $1.38 \mathrm{E}-06$ & $\mathrm{Sr} 90$ \\
$\mathrm{~S} 90$ & $2.99 \mathrm{E}-01$ & $2.26 \mathrm{E}-02$ & $6.76 \mathrm{E}-03$ & \\
\hline
\end{tabular}

Reference: SourceSRTC.xls, Worksheet "Dairy", columns L and Q

Table 7. Derived Response Levels, dairy pathway

\begin{tabular}{cccc}
\hline Radionuclide Group & $\begin{array}{l}\text { Radionuclide Group } \\
\text { concentration }\left(\mathrm{Ci} \mathrm{L}^{-1}\right) \\
\text { per unit deposition }\end{array}$ & $\begin{array}{c}\text { DIL (Bq L } \\
\text { Pu-238 + Pu-239 + Am-241 }\end{array}$ & $\begin{array}{l}\text { Deposition of mix corresponding } \\
\left.\text { to 1 DIL (Ci m }{ }^{-2}\right)\end{array}$ \\
Ce144 & $4.66 \mathrm{E}-07$ & 2 & $1.16 \mathrm{E}-04$ \\
$\mathrm{Cm} 244$ & $1.17 \mathrm{E}-09$ & 500 & $1.16 \mathrm{E}+01$ \\
$\mathrm{Cs} 137$ & $8.33 \mathrm{E}-08$ & 2 & $6.49 \mathrm{E}-04$ \\
$\mathrm{~Np} 237$ & $1.92 \mathrm{E}-02$ & 1200 & $1.69 \mathrm{E}-06$ \\
$\mathrm{Pu}-241$ & $4.30 \mathrm{E}-10$ & 4 & $2.51 \mathrm{E}-01$ \\
$\mathrm{Sr} 90$ & $1.38 \mathrm{E}-06$ & 120 & $2.36 \mathrm{E}-03$ \\
\hline
\end{tabular}

Reference: SourceSRTC.xls, Worksheet "Dairy", columns Q to S 
For this release, $\mathrm{Sr}^{90}$ is the most limiting radionuclide, with concentrations in milk reaching a DIL when the total deposition is equal to $6.40 \mathrm{E}-07 \mathrm{Ci} \mathrm{m}^{-2}$ of the particulate mix. Other radionuclides require higher depositions before the concentration in milk reaches their respective DILs.

Depositions needed to reach a DIL are calculated in a similar manner for other pathways. Results are presented in Table 8. Details of these spreadsheet calculations are presented in Attachments B to Q.

Table 8. Derived Response Levels for ingestion pathways

\begin{tabular}{|c|c|c|c|c|c|c|c|}
\hline \multirow[t]{2}{*}{ Pathway } & \multicolumn{7}{|c|}{ Deposition of mix $\left(\mathrm{Ci} \mathrm{m}^{-2}\right)$ corresponding to $1 \mathrm{DIL}$ of nuclide group } \\
\hline & $\begin{array}{r}\text { Pu-238+ } \\
\text { Pu-239 + } \\
\text { Am-241 }\end{array}$ & $\mathrm{Ce} 144$ & $\mathrm{Cm} 244$ & Cs137 & Np237 & Pu-241 & Sr90 \\
\hline Milk & $1.16 \mathrm{E}-04$ & $1.16 \mathrm{E}+01$ & $6.49 \mathrm{E}-04$ & $1.69 \mathrm{E}-06$ & $2.51 \mathrm{E}-01$ & $2.36 \mathrm{E}-03$ & 6.40E-07 \\
\hline Egg contents & $2.61 \mathrm{E}-05$ & $9.43 \mathrm{E}+02$ & NA & $8.13 \mathrm{E}-06$ & NA & $1.27 \mathrm{E}-03$ & $2.19 \mathrm{E}-06$ \\
\hline Beef & $8.88 \mathrm{E}-06$ & $1.88 \mathrm{E}+01$ & $7.00 \mathrm{E}-05$ & $2.87 \mathrm{E}-07$ & $1.35 \mathrm{E}-03$ & $2.80 \mathrm{E}-04$ & 2.41E-07 \\
\hline Veal & 4.68E-07 & NA & NA & $2.25 \mathrm{E}-07$ & NA & $8.78 \mathrm{E}-06$ & $6.06 E-08$ \\
\hline Sheep & $5.78 \mathrm{E}-06$ & $8.58 \mathrm{E}+00$ & NA & $3.87 \mathrm{E}-07$ & NA & $1.36 \mathrm{E}-04$ & 2.21E-07 \\
\hline Lamb & $2.42 \mathrm{E}-07$ & NA & NA & $1.58 \mathrm{E}-07$ & NA & $4.87 \mathrm{E}-06$ & $3.16 \mathrm{E}-08$ \\
\hline Pork & $3.65 \mathrm{E}-06$ & $9.21 \mathrm{E}+00$ & NA & $1.47 \mathrm{E}-07$ & NA & $8.58 \mathrm{E}-05$ & 1.19E-07 \\
\hline Poultry & $3.47 \mathrm{E}-06$ & $8.04 \mathrm{E}+00$ & NA & $1.23 \mathrm{E}-07$ & NA & $7.99 \mathrm{E}-05$ & 2.07E-06 \\
\hline Fish & $3.73 E-08$ & $9.38 \mathrm{E}-02$ & $3.50 \mathrm{E}-07$ & $5.39 \mathrm{E}-08$ & $3.39 \mathrm{E}-04$ & $6.99 \mathrm{E}-07$ & $2.41 \mathrm{E}-07$ \\
\hline $\begin{array}{l}\text { Produce-direct } \\
\text { deposition }\end{array}$ & 3.91E-09 & $9.85 \mathrm{E}-03$ & $3.67 \mathrm{E}-08$ & $3.77 \mathrm{E}-07$ & $3.55 \mathrm{E}-05$ & 7.34E-08 & $5.07 \mathrm{E}-08$ \\
\hline Produce - root uptake & $1.07 \mathrm{E}-02$ & $1.84 \mathrm{E}+02$ & $2.67 \mathrm{E}-02$ & $4.59 \mathrm{E}-04$ & $5.38 \mathrm{E}-01$ & $5.63 \mathrm{E}-01$ & $9.46 E-06$ \\
\hline Produce-soil adhesion & $2.19 \mathrm{E}-04$ & $5.51 \mathrm{E}+02$ & $2.06 \mathrm{E}-03$ & $2.11 \mathrm{E}-02$ & $1.99 \mathrm{E}+00$ & $4.11 \mathrm{E}-03$ & $2.84 \mathrm{E}-03$ \\
\hline Grain-direct deposition & $3.91 \mathrm{E}-09$ & $9.85 \mathrm{E}-03$ & $3.67 \mathrm{E}-08$ & $3.77 \mathrm{E}-07$ & $3.55 \mathrm{E}-05$ & $7.34 \mathrm{E}-08$ & $5.07 \mathrm{E}-08$ \\
\hline Grain-root uptake & $3.13 \mathrm{E}-02$ & $3.05 \mathrm{E}+01$ & $1.63 \mathrm{E}-01$ & $4.23 \mathrm{E}-04$ & $1.22 \mathrm{E}+00$ & $7.94 \mathrm{E}-01$ & $2.25 E-05$ \\
\hline 1 adhesion & $9.10 \mathrm{E}-05$ & $2.29 \mathrm{E}+02$ & 8.54E-04 & $8.77 \mathrm{E}-03$ & $8.27 \mathrm{E}-01$ & $1.71 \mathrm{E}-03$ & $1.18 \mathrm{E}-03$ \\
\hline Beverage & 1.12E-06 & $2.81 E+00$ & $1.05 \mathrm{E}-05$ & $1.08 \mathrm{E}-04$ & $1.02 \mathrm{E}-02$ & $2.10 \mathrm{E}-05$ & $1.45 \mathrm{E}-05$ \\
\hline
\end{tabular}

Reference: SourceSRTC.xls, individual pathway worksheets

Limiting depositions are bolded for each pathway in Table 8.

Limiting depositions for each pathway are ranked in Table 9 from most limiting (smallest DRL) to least limiting (largest DRL). 


\begin{tabular}{|c|c|}
\hline Pathway & $\begin{array}{l}\text { DRL } \\
\mathrm{Ci} \mathrm{m}^{-2}\end{array}$ \\
\hline Produce-direct deposition & $3.91 \mathrm{E}-09$ \\
\hline Grain-direct deposition & $3.91 \mathrm{E}-09$ \\
\hline Lamb & $3.16 \mathrm{E}-08$ \\
\hline Fish & $3.73 \mathrm{E}-08$ \\
\hline Veal & $6.06 \mathrm{E}-08$ \\
\hline Pork & $1.19 \mathrm{E}-07$ \\
\hline Sheep & $2.21 \mathrm{E}-07$ \\
\hline Beef & $2.41 \mathrm{E}-07$ \\
\hline Milk & $6.40 \mathrm{E}-07$ \\
\hline Beverage & $1.12 \mathrm{E}-06$ \\
\hline Poultry & $2.07 \mathrm{E}-06$ \\
\hline Egg contents & $2.19 \mathrm{E}-06$ \\
\hline Produce - root uptake & $9.46 \mathrm{E}-06$ \\
\hline Grain-root uptake & $2.25 \mathrm{E}-05$ \\
\hline Grain-soil adhesion & $9.10 \mathrm{E}-05$ \\
\hline Produce-soil adhesion & $2.19 \mathrm{E}-04$ \\
\hline
\end{tabular}

\section{Determination of range and area of consequences}

The HOTSPOT computer code was selected to evaluate the consequences of particulate releases (WSRC-TR-98-00392). In the following calculations, a source term equal to the total particulate activity $(2.96 \mathrm{Ci})$ is used, and the range and area affected for different meteorological parameters and DRLs found. HOTSPOT Parameters used for the SRTC release are listed in Table 10. Pasquill-Briggs parameterization is used in the HOTSPOT code.

Table 10. HOTSPOT input parameters for SRTC releases

\begin{tabular}{|c|c|c|}
\hline Parameter & Average Meteorology & $\begin{array}{l}95 \% \text { Adverse } \\
\text { Meteorology }\end{array}$ \\
\hline Release height & Ground & Ground \\
\hline Wind speed & $2.5 \mathrm{~m} / \mathrm{s}$ & $1.7 \mathrm{~m} / \mathrm{s}$ \\
\hline $\begin{array}{l}\text { Surface } \\
\text { roughness }\end{array}$ & $100 \mathrm{~cm}$ & $100 \mathrm{~cm}$ \\
\hline Stability class & $\mathrm{C}$ & $\mathrm{E}$ \\
\hline $\begin{array}{l}\text { Deposition } \\
\text { velocity }\end{array}$ & $0.1 / 1 / 10 \mathrm{~cm} / \mathrm{sec}$ & $0.1 / 1 / 10 \mathrm{~cm} / \mathrm{sec}$ \\
\hline Release duration & $60 \mathrm{~min}$ & $60 \mathrm{~min}$ \\
\hline Inversion layer & $500 \mathrm{~m}$ & $200 \mathrm{~m}$ \\
\hline
\end{tabular}




\section{Results}

The following four tables summarize ingestion pathway consequences for average and adverse meteorology. If a contour (DRL) is not exceeded and/or the area affected is insignificant, "N/E" is entered for the value. The site boundary is $0.67 \mathrm{~km}$ from the release.

Table 11. Ranges until deposition less than DRL, average meteorology, SRTC

\begin{tabular}{lcccc}
\hline Pathway & $\begin{array}{r}\text { DRL } \\
\mathrm{Ci} \mathrm{m}^{-2}\end{array}$ & $\begin{array}{c}0.1 \mathrm{~cm} \mathrm{~s}^{-1} \\
\mathrm{~km}\end{array}$ & $\begin{array}{c}1 \mathrm{~cm} \mathrm{~s}^{-1} \\
\mathrm{~km}\end{array}$ & $\begin{array}{c}10 \mathrm{~cm} \mathrm{~s}^{-1} \\
\mathrm{~km}\end{array}$ \\
\hline Produce-direct deposition & $3.91 \mathrm{E}-09$ & 1.5 & 21 & $>100$ \\
Grain-direct deposition & $3.91 \mathrm{E}-09$ & 1.5 & 21 & $>100$ \\
Lamb & $3.16 \mathrm{E}-08$ & 0.45 & 1.5 & 6 \\
Fish & $3.73 \mathrm{E}-08$ & 0.40 & 1.5 & 5 \\
Veal & $6.06 \mathrm{E}-08$ & 0.30 & 1.0 & 2.5 \\
Pork & $1.19 \mathrm{E}-07$ & 0.20 & 0.7 & 1.8 \\
Sheep & $2.21 \mathrm{E}-07$ & 0.18 & 0.5 & 1.3 \\
Beef & $2.41 \mathrm{E}-07$ & 0.15 & 0.5 & 1.2 \\
Milk & $6.40 \mathrm{E}-07$ & 0.10 & 0.3 & 0.8 \\
Beverage & $1.12 \mathrm{E}-06$ & 0.075 & 0.25 & 0.55 \\
Poultry & $2.07 \mathrm{E}-06$ & 0.055 & 0.15 & 0.4 \\
Egg contents & $2.19 \mathrm{E}-06$ & 0.055 & 0.15 & 0.4 \\
Produce - root uptake & $9.46 \mathrm{E}-06$ & 0.025 & 0.08 & 0.2 \\
Grain-root uptake & $2.25 \mathrm{E}-05$ & 0.018 & 0.05 & 0.15 \\
Grain-soil adhesion & $9.10 \mathrm{E}-05$ & $\mathrm{~N} / \mathrm{E}$ & 0.025 & 0.07 \\
Produce-soil adhesion & $2.19 \mathrm{E}-04$ & $\mathrm{~N} / \mathrm{E}$ & 0.017 & 0.05 \\
\hline
\end{tabular}

" $\mathrm{N} / \mathrm{E}$ " indicates the DRL was not exceeded.

Table 12. Areas above DRL, average meteorology, SRTC

\begin{tabular}{lcccc}
\hline Pathway & $\begin{array}{c}\mathrm{DRL} \\
\mathrm{Ci} \mathrm{m}^{-2}\end{array}$ & $\begin{array}{c}0.1 \mathrm{~cm} \mathrm{~s}^{-1} \\
\mathrm{~km}^{2}\end{array}$ & $\begin{array}{c}1 \mathrm{~cm} \mathrm{~s}^{-1} \\
\mathrm{~km}^{2}\end{array}$ & $\begin{array}{c}10 \mathrm{~cm} \mathrm{~s}^{-1} \\
\mathrm{~km}^{2}\end{array}$ \\
\hline Produce-direct deposition & $3.91 \mathrm{E}-09$ & $4.4 \mathrm{E}-01$ & $3.0 \mathrm{E}+01$ & $>6.8 \mathrm{E}+02$ \\
Grain-direct deposition & $3.91 \mathrm{E}-09$ & $4.4 \mathrm{E}-01$ & $3.0 \mathrm{E}+01$ & $>6.8 \mathrm{E}+02$ \\
Lamb & $3.16 \mathrm{E}-08$ & $5.3 \mathrm{E}-02$ & $5.1 \mathrm{E}-01$ & $5.3 \mathrm{E}+00$ \\
Fish & $3.73 \mathrm{E}-08$ & $4.5 \mathrm{E}-02$ & $4.3 \mathrm{E}-01$ & $3.7 \mathrm{E}+00$ \\
Veal & $6.06 \mathrm{E}-08$ & $2.8 \mathrm{E}-02$ & $2.7 \mathrm{E}-01$ & $1.5 \mathrm{E}+00$ \\
Pork & $1.19 \mathrm{E}-07$ & $1.4 \mathrm{E}-02$ & $1.3 \mathrm{E}-01$ & $6.7 \mathrm{E}-01$ \\
Sheep & $2.21 \mathrm{E}-07$ & $7.5 \mathrm{E}-03$ & $7.2 \mathrm{E}-02$ & $3.8 \mathrm{E}-01$ \\
Beef & $2.41 \mathrm{E}-07$ & $6.5 \mathrm{E}-03$ & $6.6 \mathrm{E}-02$ & $3.5 \mathrm{E}-01$ \\
Milk & $6.40 \mathrm{E}-07$ & N/E & $2.5 \mathrm{E}-02$ & $1.4 \mathrm{E}-01$ \\
Beverage & $1.12 \mathrm{E}-06$ & N/E & $1.4 \mathrm{E}-02$ & $8.2 \mathrm{E}-02$ \\
Poultry & $2.07 \mathrm{E}-06$ & N/E & $7.7 \mathrm{E}-03$ & $4.6 \mathrm{E}-02$ \\
Egg contents & $2.19 \mathrm{E}-06$ & N/E & $7.3 \mathrm{E}-03$ & $4.4 \mathrm{E}-02$ \\
Produce - root uptake & $9.46 \mathrm{E}-06$ & N/E & N/E & $1.1 \mathrm{E}-02$ \\
Grain-root uptake & $2.25 \mathrm{E}-05$ & N/E & N/E & $4.8 \mathrm{E}-03$ \\
Grain-soil adhesion & $9.10 \mathrm{E}-05$ & N/E & N/E & N/E \\
Produce-soil adhesion & $2.19 \mathrm{E}-04$ & N/E & N/E & N/E \\
\hline
\end{tabular}

"N/E" indicates the area affected is insignificant. 
Table 13. Ranges until deposition less than DRL, $95 \%$ adverse meteorology, SRTC

\begin{tabular}{lrccc}
\hline Pathway & $\begin{array}{r}\mathrm{DRL} \\
\mathrm{Ci} \mathrm{m}\end{array}$ & $\begin{array}{c}0.1 \mathrm{~cm} \mathrm{~s}^{-1} \\
\mathrm{~km}\end{array}$ & $\begin{array}{c}1 \mathrm{~cm} \mathrm{~s}^{-1} \\
\mathrm{~km}\end{array}$ & $\begin{array}{c}10 \mathrm{~cm} \mathrm{~s}^{-1} \\
\mathrm{~km}\end{array}$ \\
\hline Produce-direct deposition & $3.91 \mathrm{E}-09$ & 15 & $>100$ & 20 \\
Grain-direct deposition & $3.91 \mathrm{E}-09$ & 15 & $>100$ & 20 \\
Lamb & $3.16 \mathrm{E}-08$ & 2 & 10 & 4.5 \\
Fish & $3.73 \mathrm{E}-08$ & 1.7 & 8 & 4.0 \\
Veal & $6.06 \mathrm{E}-08$ & 1.1 & 5 & 3.0 \\
Pork & $1.19 \mathrm{E}-07$ & 0.8 & 3 & 2.0 \\
Sheep & $2.21 \mathrm{E}-07$ & 0.5 & 2 & 1.5 \\
Beef & $2.41 \mathrm{E}-07$ & 0.5 & 2 & 1.5 \\
Milk & $6.40 \mathrm{E}-07$ & 0.3 & 1.0 & 0.9 \\
Beverage & $1.12 \mathrm{E}-06$ & 0.2 & 0.7 & 0.7 \\
Poultry & $2.07 \mathrm{E}-06$ & 0.15 & 0.5 & 0.5 \\
Egg contents & $2.19 \mathrm{E}-06$ & 0.15 & 0.5 & 0.5 \\
Produce - root uptake & $9.46 \mathrm{E}-06$ & 0.07 & 0.2 & 0.3 \\
Grain-root uptake & $2.25 \mathrm{E}-05$ & 0.05 & 0.15 & 0.2 \\
Grain-soil adhesion & $9.10 \mathrm{E}-05$ & 0.02 & 0.07 & 0.1 \\
Produce-soil adhesion & $2.19 \mathrm{E}-04$ & 0.015 & 0.045 & 0.08 \\
\hline
\end{tabular}

Table 14. Areas above DRL, $95 \%$ adverse meteorology, SRTC

\begin{tabular}{lcccc}
\hline Pathway & $\begin{array}{r}\mathrm{DRL} \\
\mathrm{Ci} \mathrm{m}\end{array}$ & $\begin{array}{c}0.1 \mathrm{~cm} \mathrm{~s}^{-1} \\
\mathrm{~km}^{2}\end{array}$ & $\begin{array}{c}1 \mathrm{~cm} \mathrm{~s}^{-1} \\
\mathrm{~km}^{2}\end{array}$ & $\begin{array}{c}10 \mathrm{~cm} \mathrm{~s}^{-1} \\
\mathrm{~km}^{2}\end{array}$ \\
\hline Produce-direct deposition & $3.91 \mathrm{E}-09$ & $8.3 \mathrm{E}+00$ & $>4.5 \mathrm{E}+02$ & $2.4 \mathrm{E}+01$ \\
Grain-direct deposition & $3.91 \mathrm{E}-09$ & $8.3 \mathrm{E}+00$ & $>4.5 \mathrm{E}+02$ & $2.4 \mathrm{E}+01$ \\
Lamb & $3.16 \mathrm{E}-08$ & $3.4 \mathrm{E}-01$ & $5.9 \mathrm{E}+00$ & $1.9 \mathrm{E}+00$ \\
Fish & $3.73 \mathrm{E}-08$ & $2.9 \mathrm{E}-01$ & $4.0 \mathrm{E}+00$ & $1.6 \mathrm{E}+00$ \\
Veal & $6.06 \mathrm{E}-08$ & $1.6 \mathrm{E}-01$ & $2.1 \mathrm{E}+00$ & $9.9 \mathrm{E}-01$ \\
Pork & $1.19 \mathrm{E}-07$ & $7.1 \mathrm{E}-02$ & $8.6 \mathrm{E}-01$ & $5.2 \mathrm{E}-01$ \\
Sheep & $2.21 \mathrm{E}-07$ & $3.5 \mathrm{E}-02$ & $4.0 \mathrm{E}-01$ & $3.0 \mathrm{E}-01$ \\
Beef & $2.41 \mathrm{E}-07$ & $3.2 \mathrm{E}-02$ & $3.6 \mathrm{E}-01$ & $2.7 \mathrm{E}-01$ \\
Milk & $6.40 \mathrm{E}-07$ & $1.1 \mathrm{E}-02$ & $1.1 \mathrm{E}-01$ & $1.1 \mathrm{E}-01$ \\
Beverage & $1.12 \mathrm{E}-06$ & $6.0 \mathrm{E}-03$ & $6.0 \mathrm{E}-02$ & $7.0 \mathrm{E}-02$ \\
Poultry & $2.07 \mathrm{E}-06$ & $3.1 \mathrm{E}-03$ & $3.1 \mathrm{E}-02$ & $4.2 \mathrm{E}-02$ \\
Egg contents & $2.19 \mathrm{E}-06$ & $2.9 \mathrm{E}-03$ & $2.8 \mathrm{E}-02$ & $4.0 \mathrm{E}-02$ \\
Produce - root uptake & $9.46 \mathrm{E}-06$ & N/E & $6.1 \mathrm{E}-03$ & $1.2 \mathrm{E}-02$ \\
Grain-root uptake & $2.25 \mathrm{E}-05$ & N/E & $2.6 \mathrm{E}-03$ & $6.1 \mathrm{E}-03$ \\
Grain-soil adhesion & $9.10 \mathrm{E}-05$ & N/E & N/E & $2.0 \mathrm{E}-03$ \\
Produce-soil adhesion & $2.19 \mathrm{E}-04$ & N/E & N/E & N/E \\
\hline
\end{tabular}

$\mathrm{N} / \mathrm{E}$ " indicates the area affected is insignificant.

Data output sheets for particulate dispersion/deposition calculations are provided in Attachments R to W. 


\section{References}

Food and Drug Administration. Accidental radioactive contamination of human foods and animal feeds: recommendations for state and local agencies. Rockville, MD: FDA; August 13, 1998.

Hadlock, D. J., SRTC Bounding Accident Source Term and Consequence Assessment for Emergency Planning, Aiken, SC: Westinghouse Savannah River Company; S-CLC-A00080, Rev. 0, 4/14/97.

Hadlock, D. J., Emergency Preparedness Hazards Assessment for the SRTC TA, Aiken, SC: Westinghouse Savannah River Company; S-EHA-A-00001, Rev. 1, September $30,1997$.

Homann, Steven G., HOTSPOT Health Physics Codes for the PC, UCRL-MA-106315, Lawrence Livermore National Laboratory, University of California, Livermore, CA, March 1994.

Marx, D. R., Foodstuff Concentrations Following a SRTC Tritium Oxide Release (U), Aiken, SC; Westinghouse Savannah River Company; WSRC-TR-99-00117, Rev. 0; April 1999.

Thompson, J. M. Ingestion Pathway Transfer Factors for Particulates, Aiken, SC; Westinghouse Savannah River Company; S-CLC-G-00179, Rev. 0; March 1999.

Thompson, J. M. Ingestion Pathway Transfer Factors for Plutonium and Americium (U), Aiken, SC; Westinghouse Savannah River Company; WSRC-TR-99-00005, Rev. 0; January 1999.

Thompson, J. M. Selection of Models for Ingestion Pathway and Relocation (U), Aiken, SC; Westinghouse Savannah River Company; WSRC-TR-98-00392, Rev. 2; February, 1999.

Thompson, J. M. Total Airborne Release for SRTC Accident Scenario 1-RD-3 (U), Aiken, SC; Westinghouse Savannah River Company; S-CLC-A-00104, Rev. 0; March 1999. 


\section{Attachments}

Attachment A

Source term and areal concentrations spreadsheet calculations

Attachment B

Contamination of dairy products spreadsheet calculations

\section{Attachment C}

Contamination of egg contents spreadsheet calculations

Attachment D

Contamination of beef spreadsheet calculations

Attachment E

Contamination of veal spreadsheet calculations

Attachment F

Contamination of sheep spreadsheet calculations

Attachment G

Contamination of lamb spreadsheet calculations

Attachment $\mathrm{H}$

Contamination of pork spreadsheet calculations

Attachment I

Contamination of poultry spreadsheet calculations

Attachment J

Contamination of fish spreadsheet calculations

Attachment $\mathrm{K}$

External contamination of produce from direct deposition spreadsheet calculations

Attachment L

Internal contamination of produce from root uptake spreadsheet calculations

Attachment $\mathrm{M}$

External contamination of leafy vegetables during harvest spreadsheet calculations

Attachment $\mathrm{N}$

External contamination of grain spreadsheet calculations

Attachment $\mathrm{O}$

Internal contamination of grain by root uptake spreadsheet calculations 
Attachment $\mathbf{P}$

External contamination of grain during harvest spreadsheet calculations

Attachment Q

Contamination of beverages spreadsheet calculations

Attachment R

HOTSPOT results - average meteorology, $0.1 \mathrm{~cm} \mathrm{~s}^{-1}$

Attachment $S$

HOTSPOT results - average meteorology, $1.0 \mathrm{~cm} \mathrm{~s}^{-1}$

Attachment T

HOTSPOT results - average meteorology, $10 \mathrm{~cm} \mathrm{~s}^{-1}$

Attachment U

HOTSPOT results - adverse meteorology, $0.1 \mathrm{~cm} \mathrm{~s}^{-1}$

Attachment V

HOTSPOT results - adverse meteorology, $1.0 \mathrm{~cm} \mathrm{~s}^{-1}$

Attachment W

HOTSPOT results - adverse meteorology, $10 \mathrm{~cm} \mathrm{~s}^{-1}$ 


\section{Attachment A}

Source term and areal concentrations spreadsheet calculations 


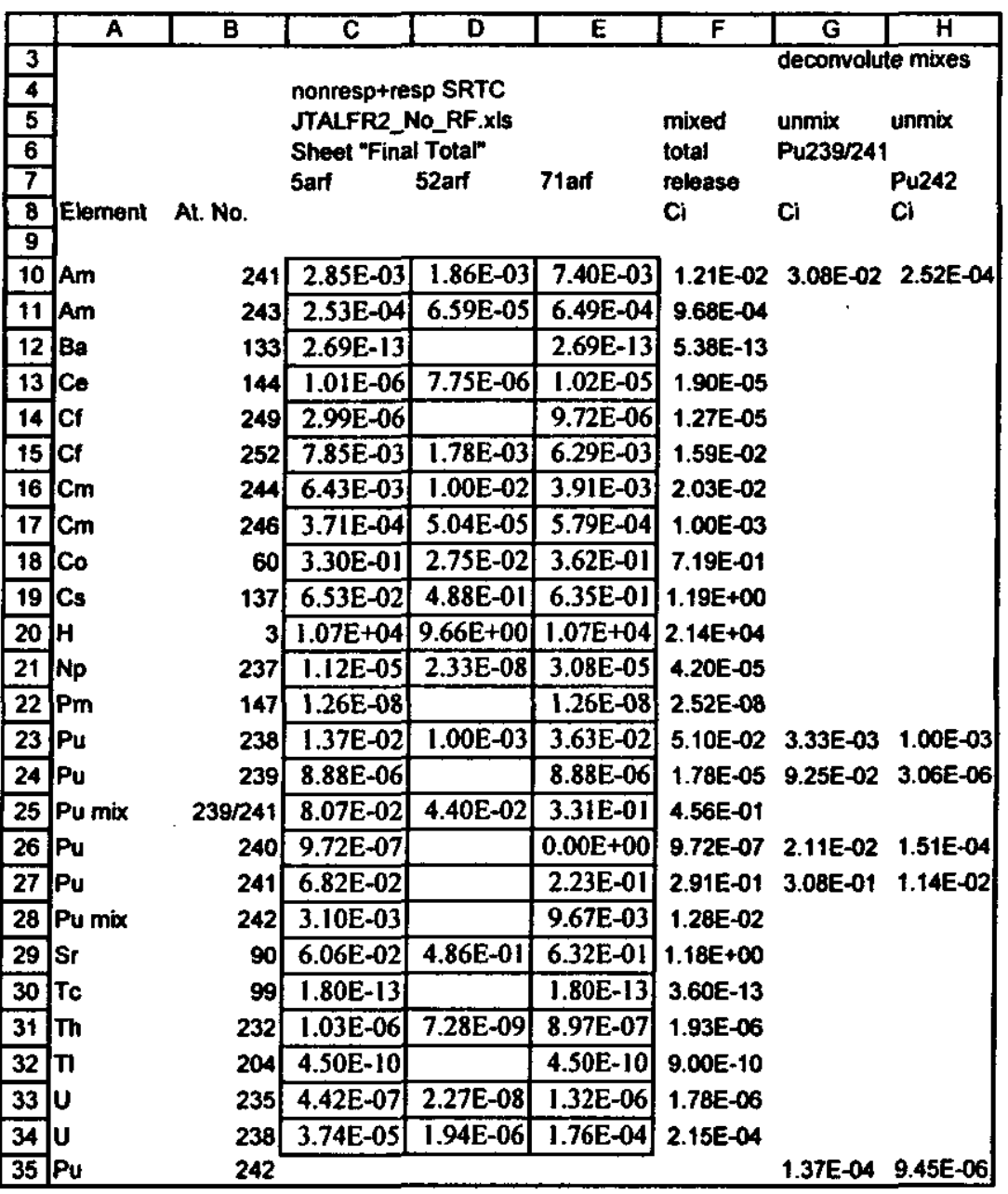


SourceSRTC.xls

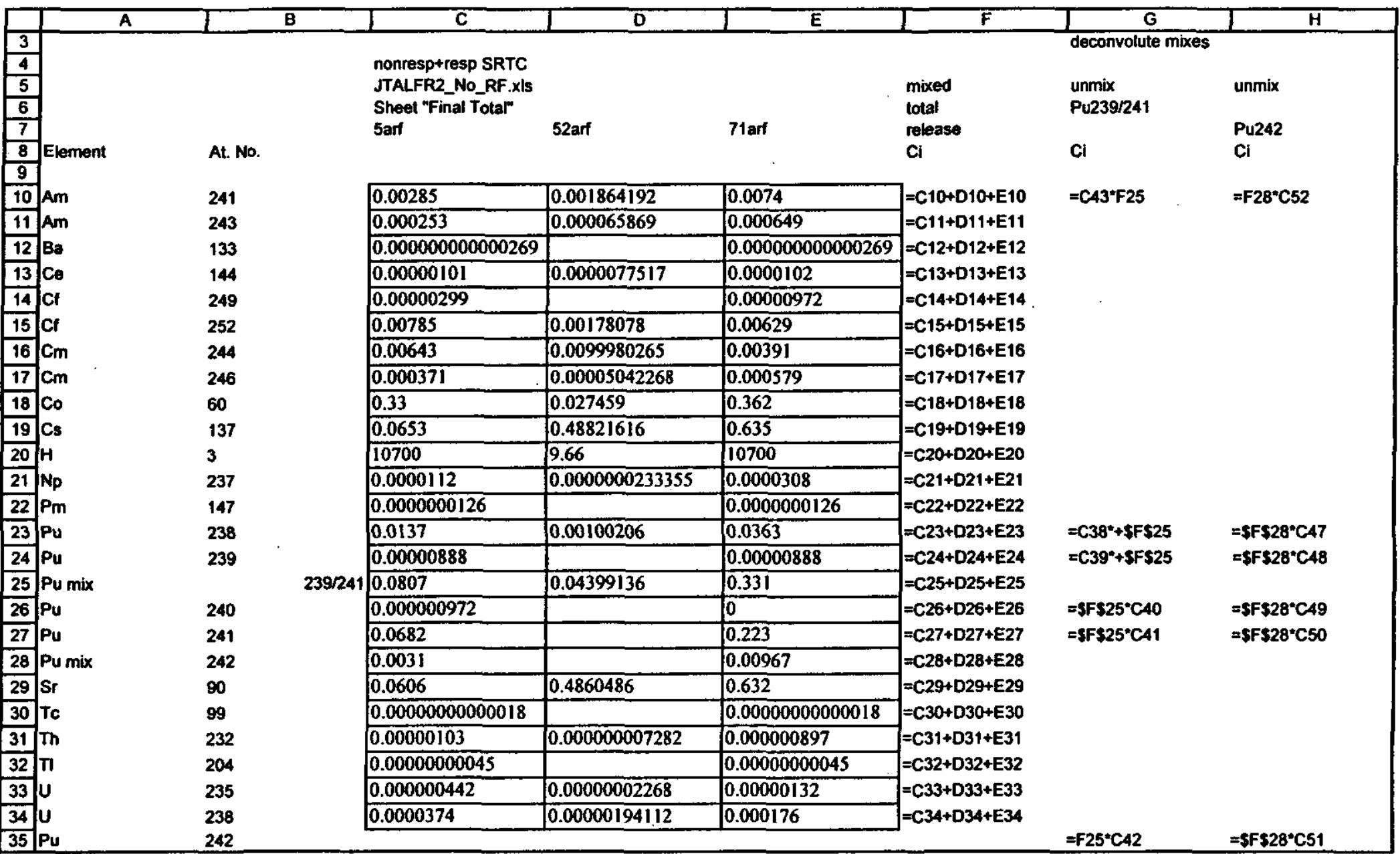


SourceSRTC.xIs

\begin{tabular}{|c|c|c|c|}
\hline & $A$ & B & C \\
\hline 36 & \multicolumn{3}{|c|}{ Pu 239/241 mix activity fractions } \\
\hline 37 & \multicolumn{3}{|c|}{ nuclide $\quad$ activity frac } \\
\hline 38 & $\mathrm{Pu}$ & 238 & 0.0073 \\
\hline 39 & Pu & 239 & 0.2029 \\
\hline 40 & Pu & 240 & 0.0462 \\
\hline 41 & $\mathrm{Pu}$ & 241 & 0.6761 \\
\hline 42 & Pu & 242 & 0.0003 \\
\hline 43 & Am & 241 & 0.0675 \\
\hline 44 & & & \\
\hline 45 & \multicolumn{3}{|c|}{ Pu 242 mix activity fractions } \\
\hline 46 & nuclide & & tivity frac \\
\hline 47 & Pu & 238 & 0.0786 \\
\hline 48 & $\mathrm{Pu}$ & 239 & 0.00024 \\
\hline 49 & $\mathrm{Pu}$ & 240 & 0.01184 \\
\hline 50 & Pu & 241 & 0.88886 \\
\hline 51 & $\mathrm{Pu}$ & 242 & 0.00074 \\
\hline 52 & Am & 241 & 0.01977 \\
\hline
\end{tabular}

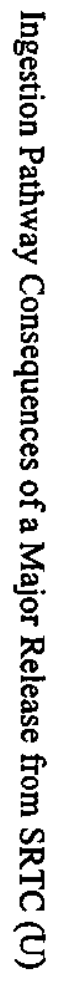

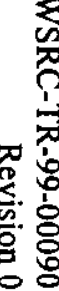

Nonresp + resp 
SourceSRTC.xls

\begin{tabular}{|c|c|c|c|}
\hline & $\bar{A}$ & $\mathbf{B}$ & $\overline{\mathbf{C}}$ \\
\hline 36 & Pu $239 / 241$ mix activity fractions & & \\
\hline 37 & nuclide & & activity frac \\
\hline 38 & Pu & 238 & 0.0073 \\
\hline 39 & $\mathrm{Pu}$ & 239 & 0.2029 \\
\hline 40 & Pu & 240 & 0.0462 \\
\hline 41 & $\mathrm{Pu}$ & 241 & 0.6761 \\
\hline 42 & $\mathrm{Pu}$ & 242 & 0.0003 \\
\hline 43 & Am & 241 & 0.0675 \\
\hline 44 & & & \\
\hline 45 & Pu 242 mix activity fractions & & \\
\hline 46 & nuclide & & activity frac \\
\hline 47 & Pu & 238 & 0.0786 \\
\hline 48 & Pu & 239 & 0.00024 \\
\hline 49 & Pu & 240 & 0.01184 \\
\hline 50 & Pu & 241 & 0.88886 \\
\hline 51 & $\mathrm{Pu}$ & 242 & 0.00074 \\
\hline 52 & Am & 241 & 0.01977 \\
\hline
\end{tabular}

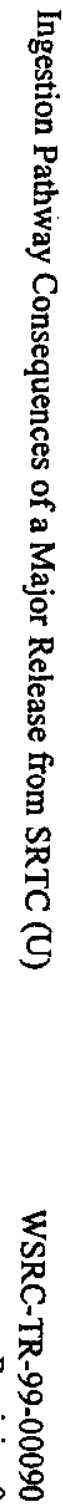

Nonresp + resp 
SourceSRTC.xls

\begin{tabular}{|c|c|c|c|c|c|c|c|}
\hline & J & $\bar{k}$ & $\mathbf{L}$ & $M$ & $\bar{N}$ & $\bar{P}$ & $\mathbf{R}$ \\
\hline 3 & & & & & & & Areal Con \\
\hline 4 & & & pre-DR & & DR'd & & per unit \\
\hline 5 & & & unmixed & & unmixed & & deposition \\
\hline 6 & & & total & & total & particulate & \\
\hline 7 & & & release & & release & release & particulate \\
\hline 8 & Element & At. No. & $\mathrm{Ci}$ & $D R=0.75$ & $\mathrm{Ci}$ & Ci & $\mathrm{Ci} / \mathrm{m}^{2}$ \\
\hline$y$ & & & & & & & \\
\hline 10 & Am & 241 & 4.31E-02 & 0.75 & $3.23 E-02$ & $3.23 E-02$ & $1.09 E-02$ \\
\hline 11 & Am & 243 & $9.68 E-04$ & 0.75 & $7.26 \mathrm{E}-04$ & $7.26 E-04$ & 2.45E-04 \\
\hline 12 & $\mathrm{Ba}$ & 133 & $5.38 \mathrm{E}-13$ & 0.75 & $4.04 E-13$ & $4.04 E-13$ & $1.36 E-13$ \\
\hline 13 & Ce & 144 & $1.90 E-05$ & 0.75 & $1.42 E-05$ & $1.42 E-0.5$ & $4.80 E-06$ \\
\hline 14 & Cf & 249 & 1.27E-05 & 0.75 & 9.53E-06 & $9.53 E-06$ & $3.22 E-06$ \\
\hline 15 & Cf & 252 & $1.59 \mathrm{E}-02$ & 0.75 & 1.19E-02 & 1.19E-02 & 4.03E-03 \\
\hline 16 & $\mathrm{Cm}$ & 244 & 2.03E-02 & 0.75 & $1.53 E-02$ & $1.53 E-02$ & 5.15E-03 \\
\hline 17 & $\mathrm{Cm}$ & 246 & $1.00 E-03$ & 0.75 & $7.50 E-04$ & $7.50 E-04$ & 2.53E-04 \\
\hline 18 & Co & 60 & 7.19E-01 & 0.75 & $5.40 E-01$ & $5.40 E-01$ & $1.82 E-01$ \\
\hline 19 & Cs & 137 & 1.19E+00 & 0.75 & 8.91E-01 & 8.91E-01 & $3.01 E-01$ \\
\hline 20 & H & 3 & $2.14 E+04$ & 0.75 & $1.61 E+04$ & & $0.00 E+\infty$ \\
\hline 21 & Np & 237 & 4.20E-05 & 0.75 & 3.15E-05 & 3.15E-05 & $1.06 E-05$ \\
\hline 22 & Pm & 147 & $2.52 E-08$ & 0.75 & $1.89 E-08$ & 1.89E-08 & $6.38 \mathrm{E}-09$ \\
\hline 23 & Pu & 238 & $5.53 E-02$ & 0.75 & 4.15E-02 & 4.15E-02 & $1.40 E-02$ \\
\hline 24 & Pu & 239 & $9.25 E-02$ & 0.75 & $6.94 E-02$ & $6.94 E-02$ & 2.34E-02 \\
\hline 25 & & & & & & & $0.00 E+00$ \\
\hline 26 & Pu & 240 & 2.12E-02 & 0.75 & $1.59 E-02$ & $1.59 E-02$ & 5.37E-03 \\
\hline 27 & $\mathrm{Pu}$ & 241 & 6.1 tE-01 & 0.75 & $4.58 E-01$ & 4.58E-01 & $1.55 E-01$ \\
\hline 28 & Pu pure & 242 & $1.46 E-04$ & 0.75 & $1.10 E-04$ & $1.10 E-04$ & $3.70 E-05$ \\
\hline 29 & Sr & 90 & $1.18 E+00$ & 0.75 & 8.84E-01 & 8.84E-01 & 2.99E-01 \\
\hline 30 & Tc & 99 & $3.60 E-13$ & 0.75 & 2.70E-13 & $2.70 E-13$ & $9.12 E-14$ \\
\hline 31 & Th & 232 & $1.93 E-06$ & 0.75 & $1.45 \mathrm{E}-06$ & 1.45E-06 & 4.90E-07 \\
\hline 32 & $\pi$ & 204 & $9.00 \mathrm{E}-10$ & 0.75 & 6.75E-10 & $6.75 E-10$ & 2.28E-10 \\
\hline 33 & $\mathbf{U}$ & 235 & $1.78 E-06$ & 0.75 & $1.34 E-06$ & $1.34 E-06$ & 4.52E-07 \\
\hline 34 & $\mathbf{u}$ & 238 & 2.15E-04 & 0.75 & $1.62 E-04$ & 4.62E-04 & 5.45E-05 \\
\hline 35 & & & & & & $2.96 E+\infty 0$ & $1.00 E+00$ \\
\hline 36 & & & & & & & \\
\hline
\end{tabular}


SoutceSRTC.X/s

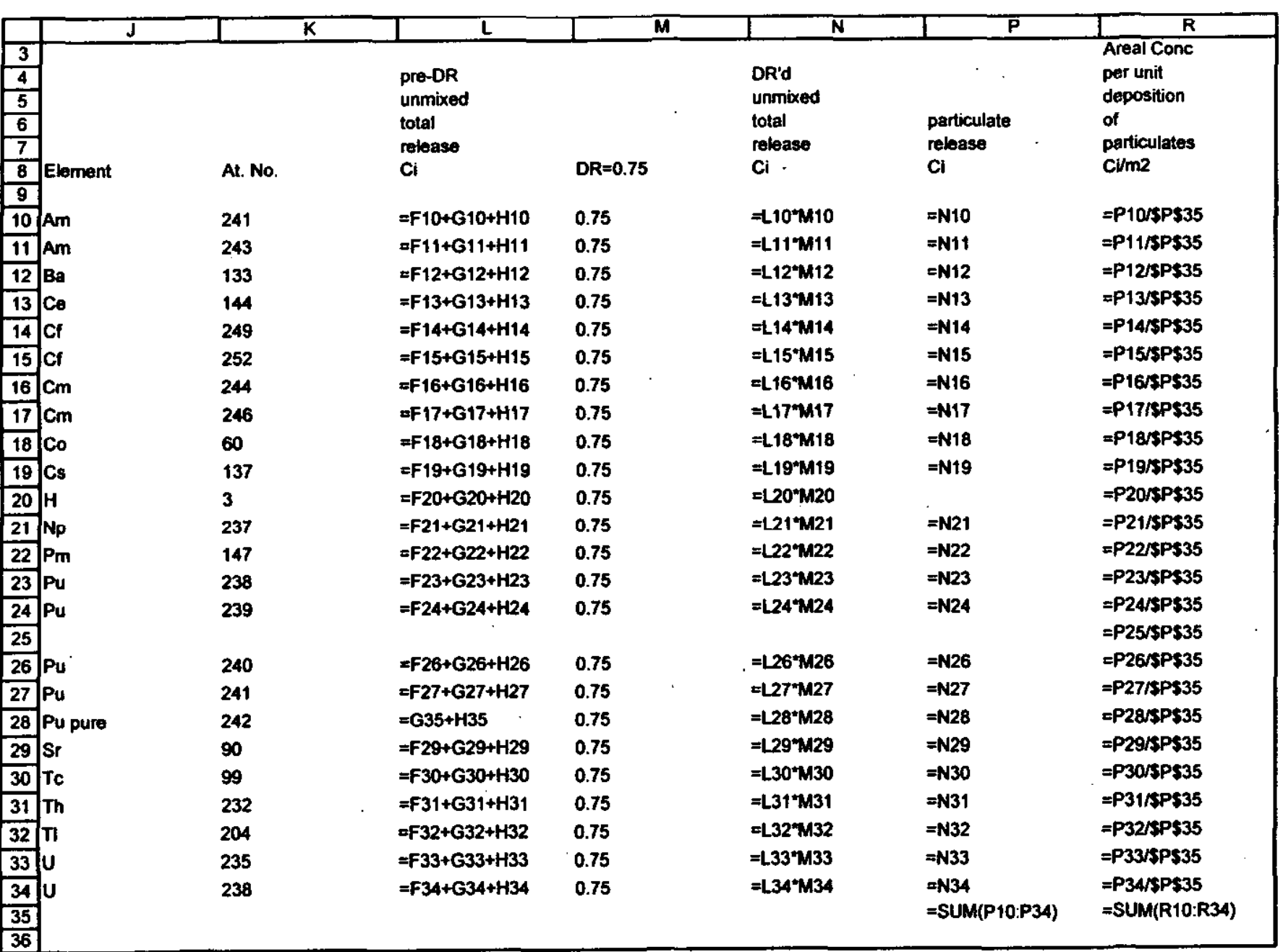




\section{Attachment B}

Contamination of dairy products spreadsheet calculations

Cell references in the formulae for the overall transfer factor (Column $L$ ) were verified in S-CLC-G-00179 and are not included in this attachment. 
SourceSRTC.xls

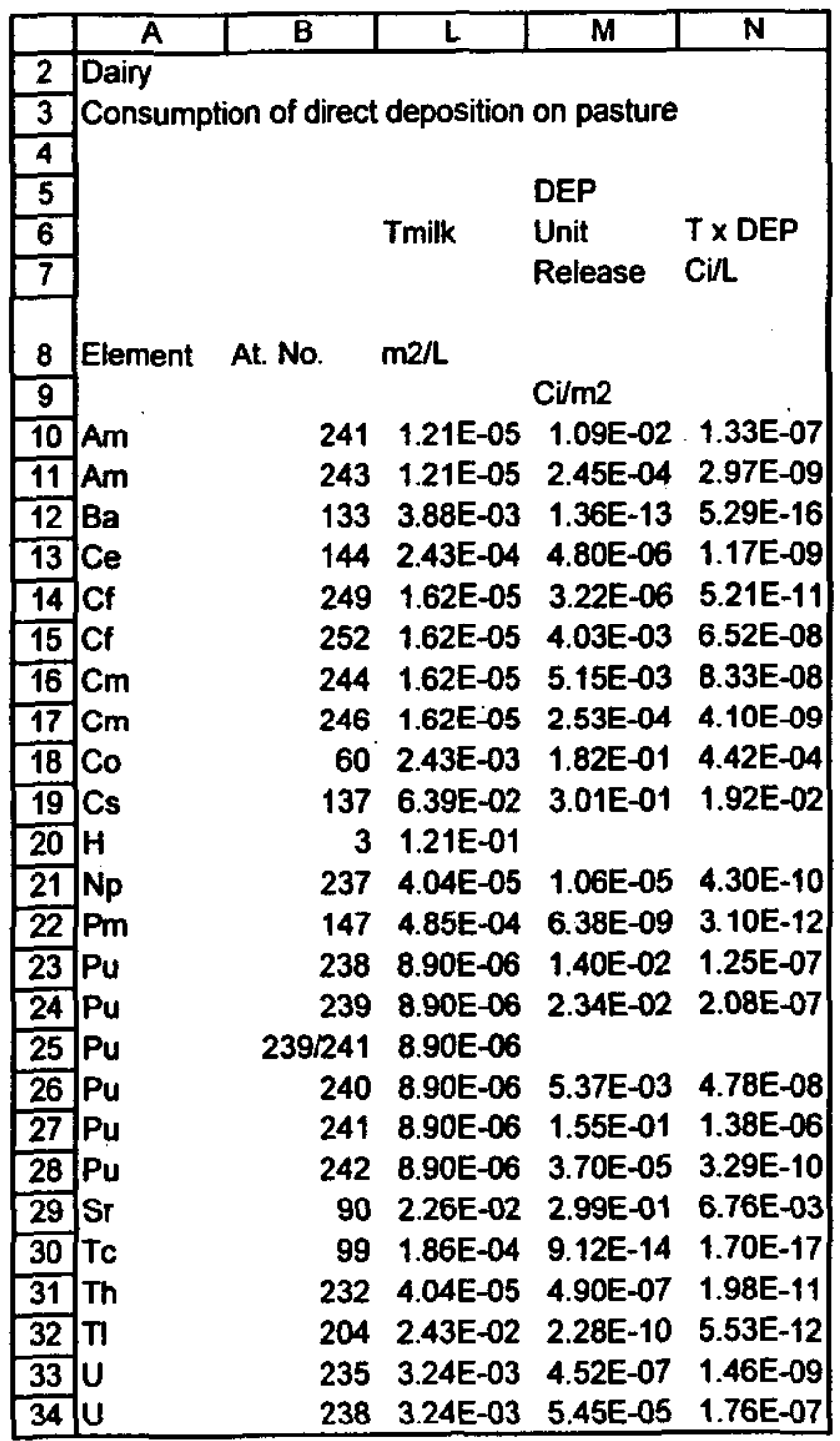


SourceSRTC.xis

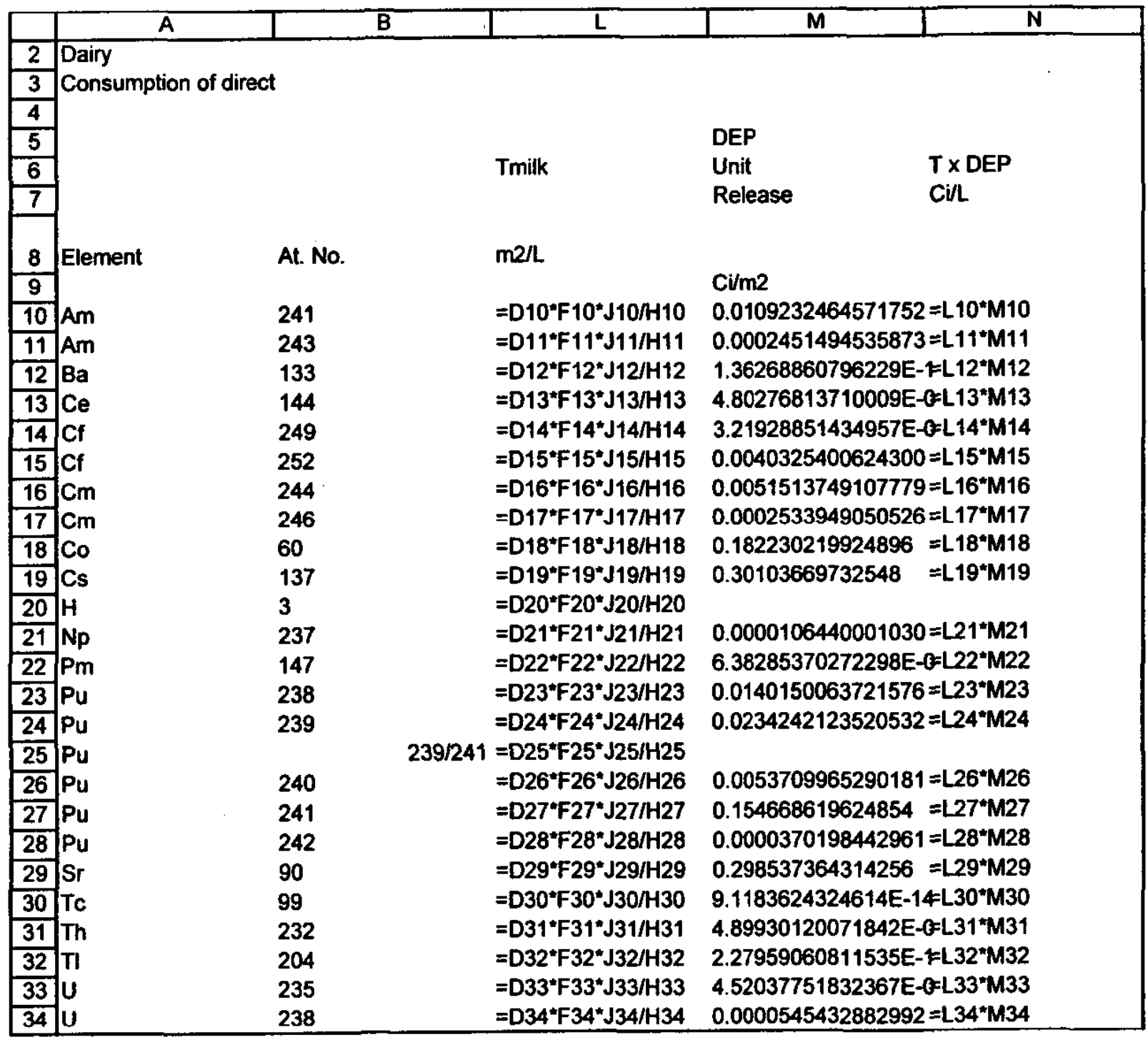


SourceSRTC.xls

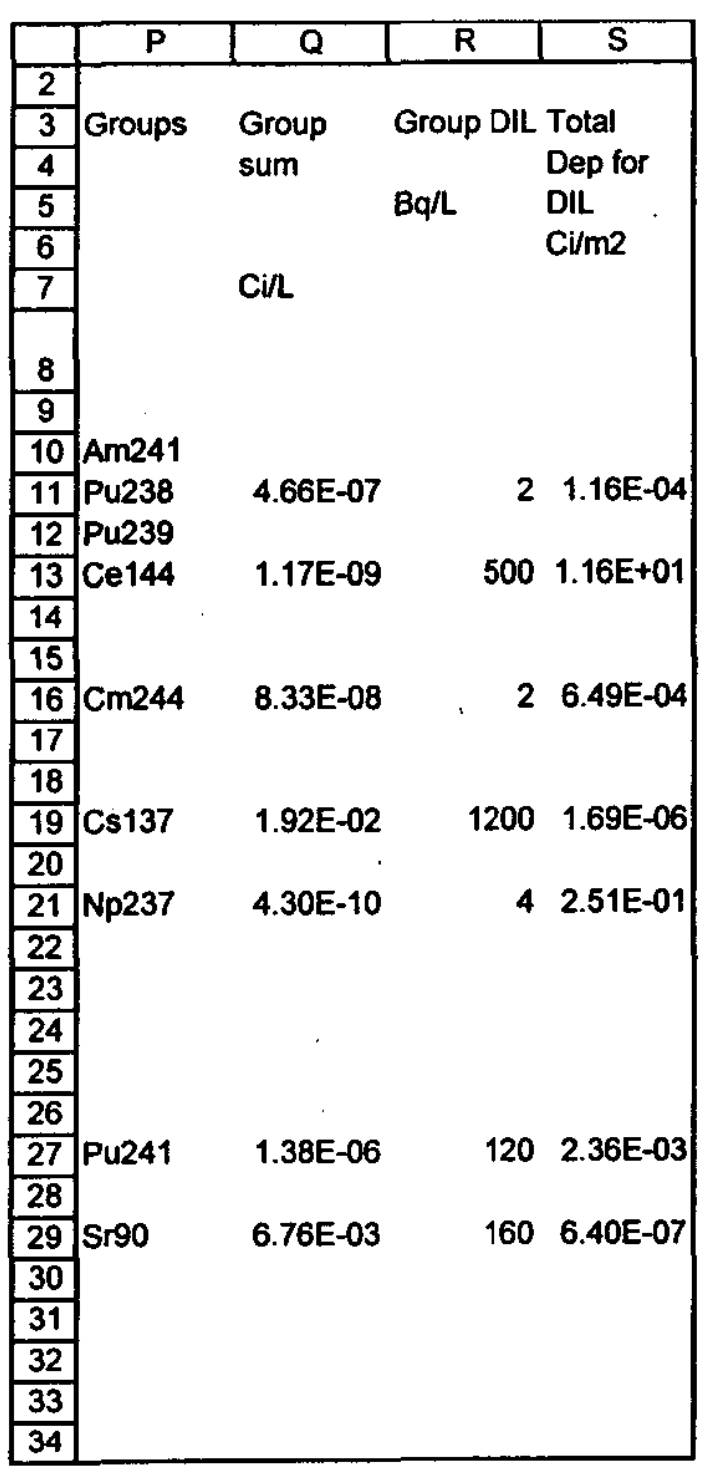

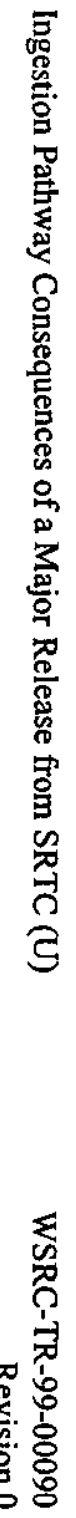


SourceSRTC.xIs

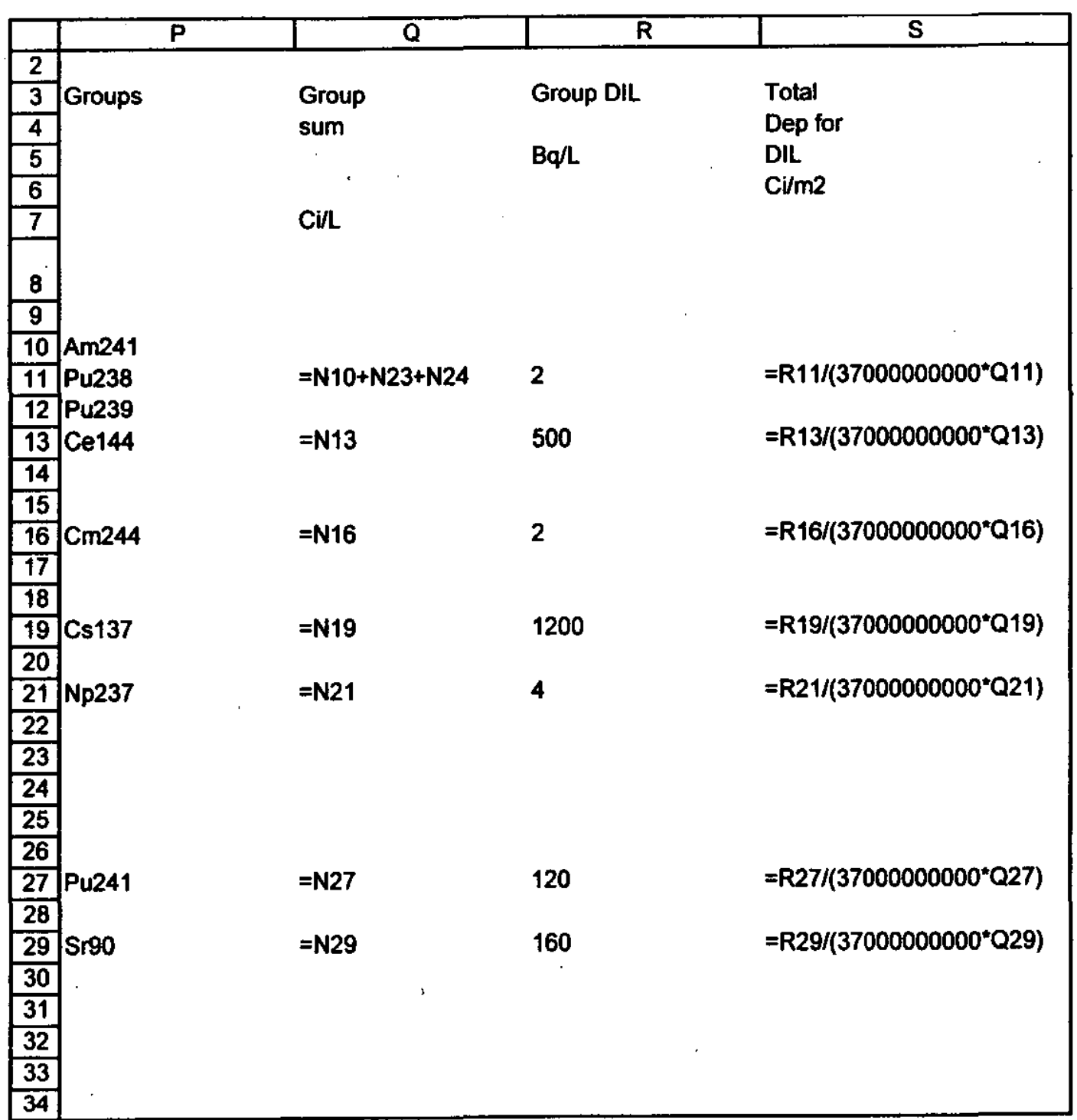

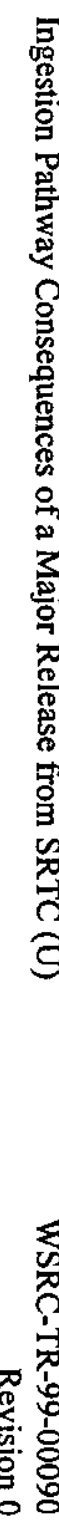




\section{Attachment C}

Contamination of egg contents spreadsheet calculations

Cell references in the formulae for the overall transfer factor (Column $\mathrm{L}$ ) were verified in S-CLC-G-00179 and are not included in this attachment. 


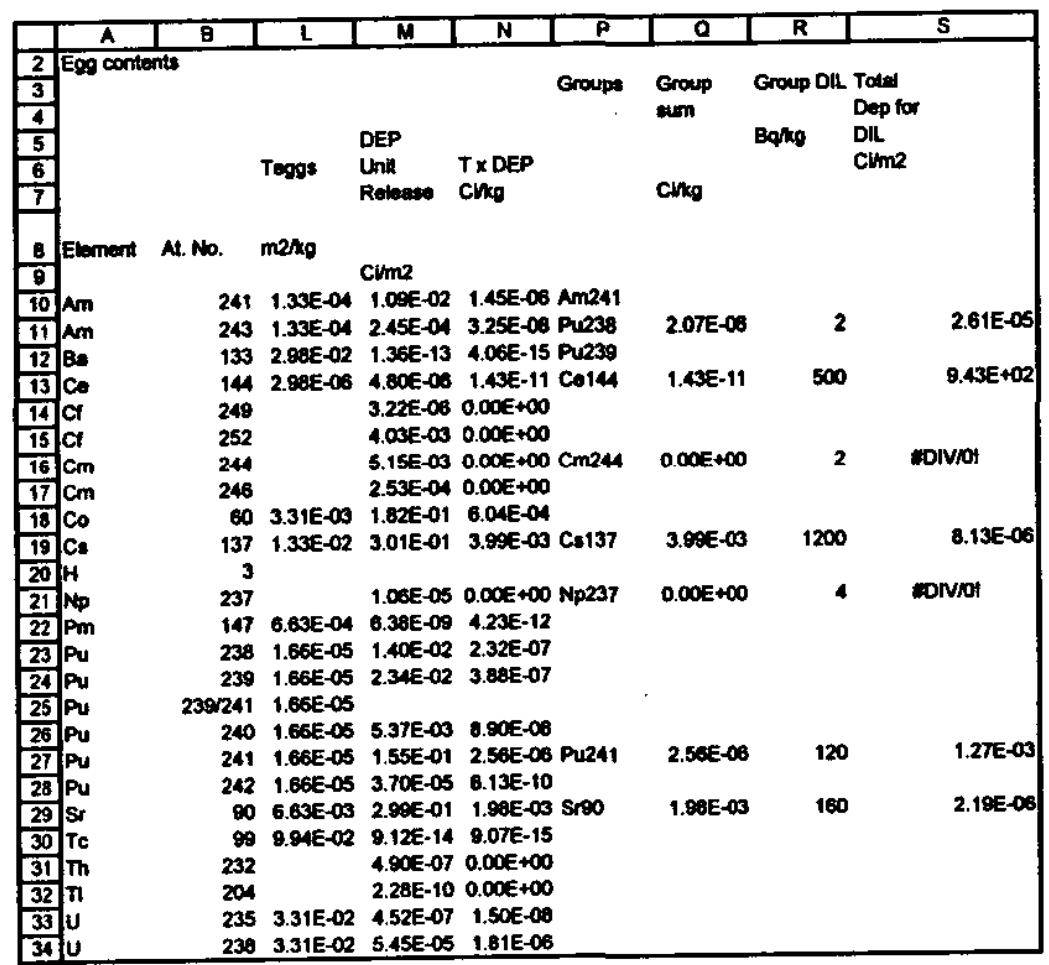




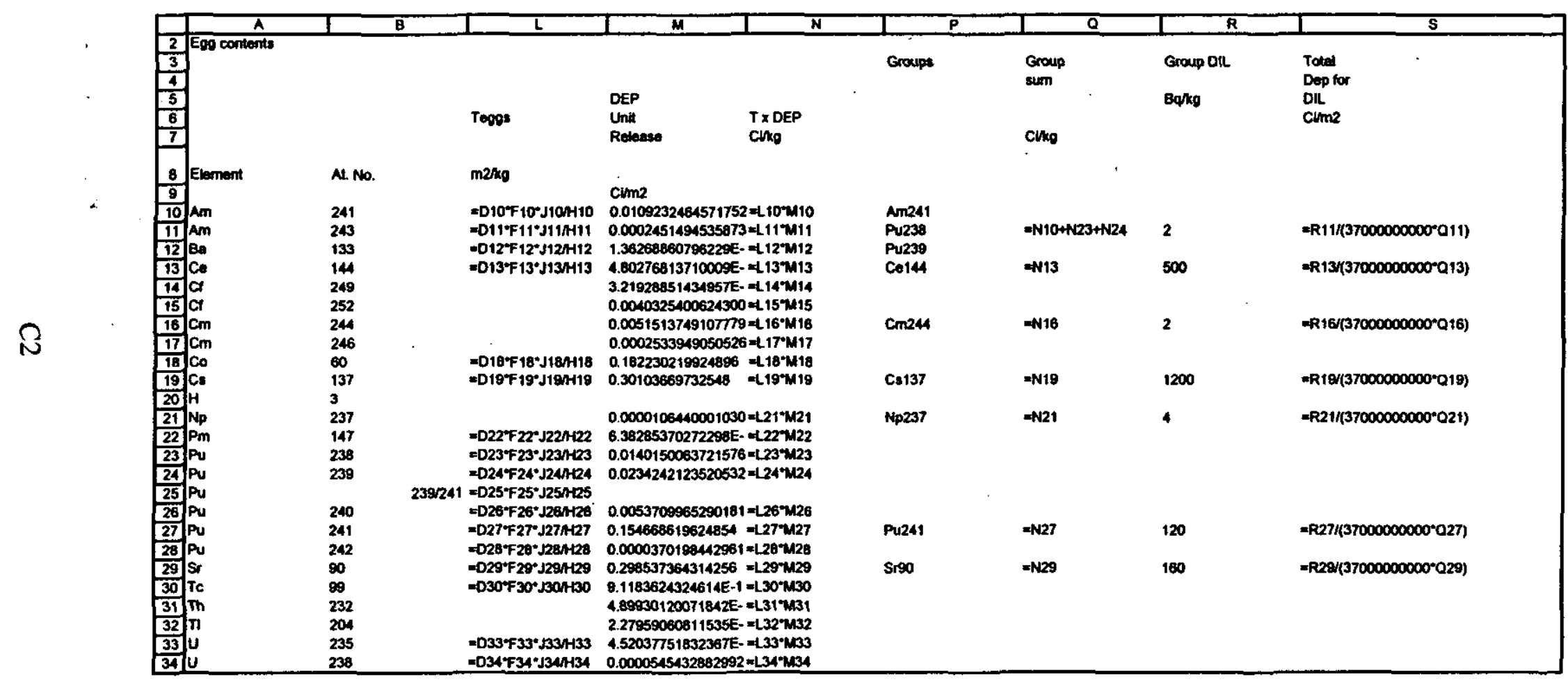




\section{Attachment D}

Contamination of beef spreadsheet calculations

Cell references in the formulae for the overall transfer factor (Column $\mathrm{L}$ ) were verified in S-CLC-G-00179 and are not included in this attachment. 


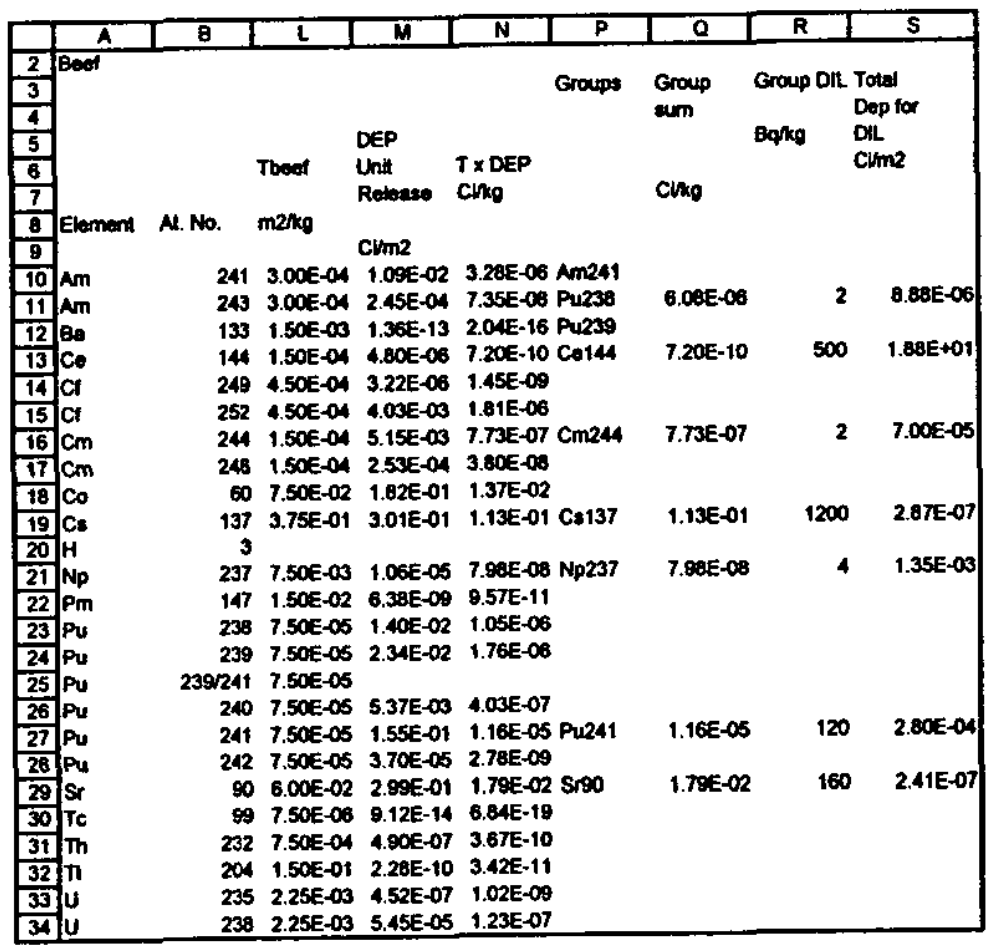




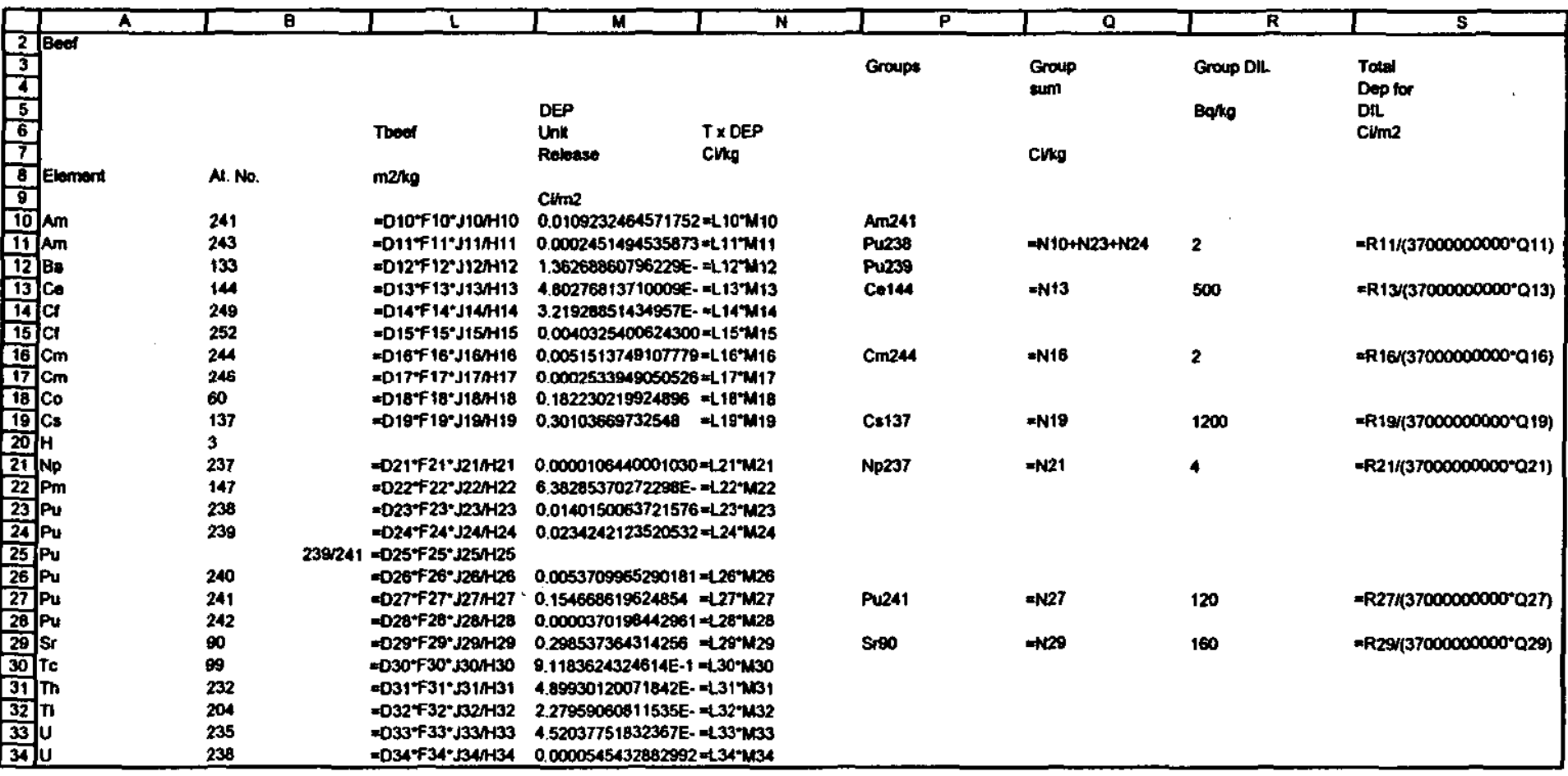




\section{Attachment E}

Contamination of veal spreadsheet calculations

Cell references in the formulae for the overall transfer factor (Column $\mathrm{L}$ ) were verified in S-CLC-G-00179 and are not included in this attachment. 


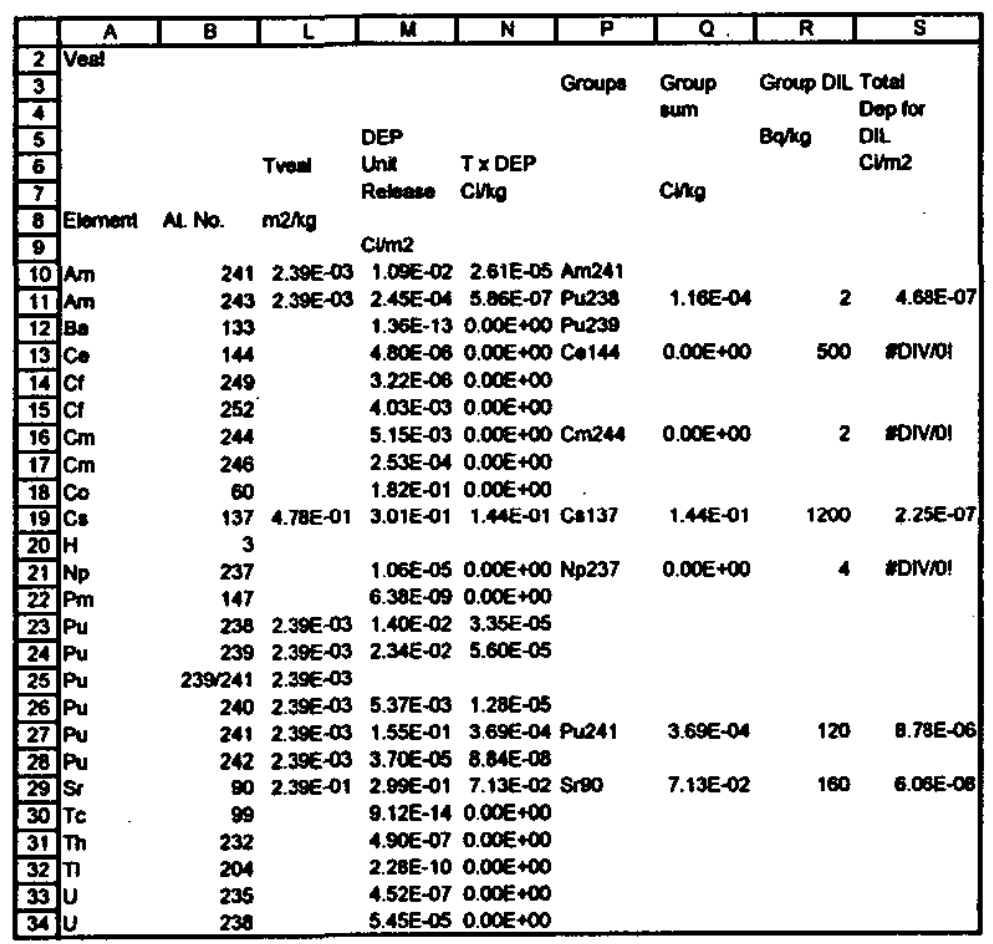




\section{Attachment $\mathbf{F}$}

Contamination of sheep spreadsheet calculations

Cell references in the formulae for the overall transfer factor (Column $\mathrm{L}$ ) were verified in S-CLC-G-00179 and are not included in this attachment. 


\begin{tabular}{|c|c|c|c|c|c|c|c|c|c|}
\hline & $\bar{A}$ & 8 & L & $\mathbf{M}$ & $\bar{N}$ & $\bar{P}$ & Q & $\bar{R}$ & 5 \\
\hline 2 & \multicolumn{9}{|l|}{ Shoep } \\
\hline$\frac{3}{4}$ & & & & & & Groups & Group & Group oll & $\begin{array}{l}\text { Totat } \\
\text { Dep for }\end{array}$ \\
\hline & & & & DEP & & & & Bopg & \\
\hline 6 & & & Tetheop & & $T \times$ DEP & & & & cumn \\
\hline 7 & & & & Revosese & Che & & Cikg & & \\
\hline 9 & Element & A. No. & mexke & Cim2 & & & & & \\
\hline 10 & Am & 241 & $3.28 E-94$ & $1.002-02$ & $3.58 E-06$ & Am241 & & & \\
\hline$\frac{11}{12}$ & $\operatorname{com}_{\mathrm{Ba}}$ & $\begin{array}{l}243 \\
133\end{array}$ & $3.28 E-04$ & $\begin{array}{l}2.45 E-04 \\
1.36 E+13\end{array}$ & $\begin{array}{l}0.04 E-00 \\
0.00 E+00\end{array}$ & $\begin{array}{l}\text { Pu238 } \\
\text { Pu239 }\end{array}$ & $9.35 E-08$ & 2 & $5.78 E-06$ \\
\hline 13 & co & 144 & $3.28 E-04$ & $4.80 E-06$ & $1.57 E-09$ & Co144 & $1.57 E-09$ & 500 & $8.58 E+\infty$ \\
\hline 14 & Ct & 249 & & $3.22 E-06$ & $0.00 E+\infty 0$ & & & & \\
\hline 15 & Cf & 252 & & $4.03 E-03$ & $0.00 E+00$ & & & & \\
\hline 16 & $\mathrm{~cm}$ & 244 & & $5.15 E-03$ & $0.00 E+\infty 0$ & $\mathrm{Cm}_{244}$ & O.00E $+\infty 0$ & 2 & DDIVIO \\
\hline 17 & $\mathrm{Cm}$ & 246 & & $2.53 E-04$ & $0.00 E+00$ & & & & \\
\hline 18 & Co & 60 & $1.02 E-01$ & $1.82 E-01$ & $1.85 E-02$ & & & & \\
\hline 19 & C. & 137 & $2.79 E-01$ & $3.01 E-01$ & 8. 39E-02 & C\&137 & 8.39E-02 & 1200 & $3.87 E-07$ \\
\hline 20 & H & $\begin{array}{r}33 \\
237\end{array}$ & & & & & $0.00 E+\infty 0$ & 4 & Porvor \\
\hline$\frac{21}{22}$ & $\begin{array}{c}N_{p} \\
P_{m}\end{array}$ & $\begin{array}{l}237 \\
147\end{array}$ & & $6.38 E-09$ & $0.00 E+\infty 0$ & & & & \\
\hline 23 & Pu & 238 & 1.54E-04 & $1.40 E-02$ & $2.16 E-06$ & & & & \\
\hline 24 & $\begin{array}{l}\text { Pu } \\
\text { Pu }\end{array}$ & $\begin{array}{r}239 \\
239241\end{array}$ & $\begin{array}{l}1.54 E-O A \\
1.54 E-04\end{array}$ & 2.34E-02 & $3.61 E-06$ & & & & \\
\hline 26 & Pu & 240 & $1.54 E-O A$ & $5.37 E-\infty 3$ & $0.27 E-07$ & & & & \\
\hline 27 & Pu & 241 & $1.5 A E-04$ & $1.55 E-01$ & $2.38 E-05$ & Pu241 & 2.38E-05 & 120 & $1.36 E-04$ \\
\hline 28 & Pu & 242 & $1.54 E-04$ & 3.70E-05 & $5.70 E-09$ & & & & \\
\hline 29 & sr & so & $6.56 E-02$ & 2.99E-01 & $1.96 E-02$ & srso & $1.96 \mathrm{E}-02$ & 160 & 2.21E-07 \\
\hline$\frac{30}{31}$ & Ic & 99 & & 9.12E-14 & $0.00 E+\infty 0$ & & & & \\
\hline$\frac{31}{32}$ & $\begin{array}{l}\text { In } \\
\pi\end{array}$ & 204 & & $2.28 E-10$ & $0.00 E+\infty$ & & & & \\
\hline & & 235 & & $4.52 E-07$ & $0.00 E+\infty 0$ & & & & \\
\hline 34 & & 238 & & 5.4 & & & & & \\
\hline
\end{tabular}




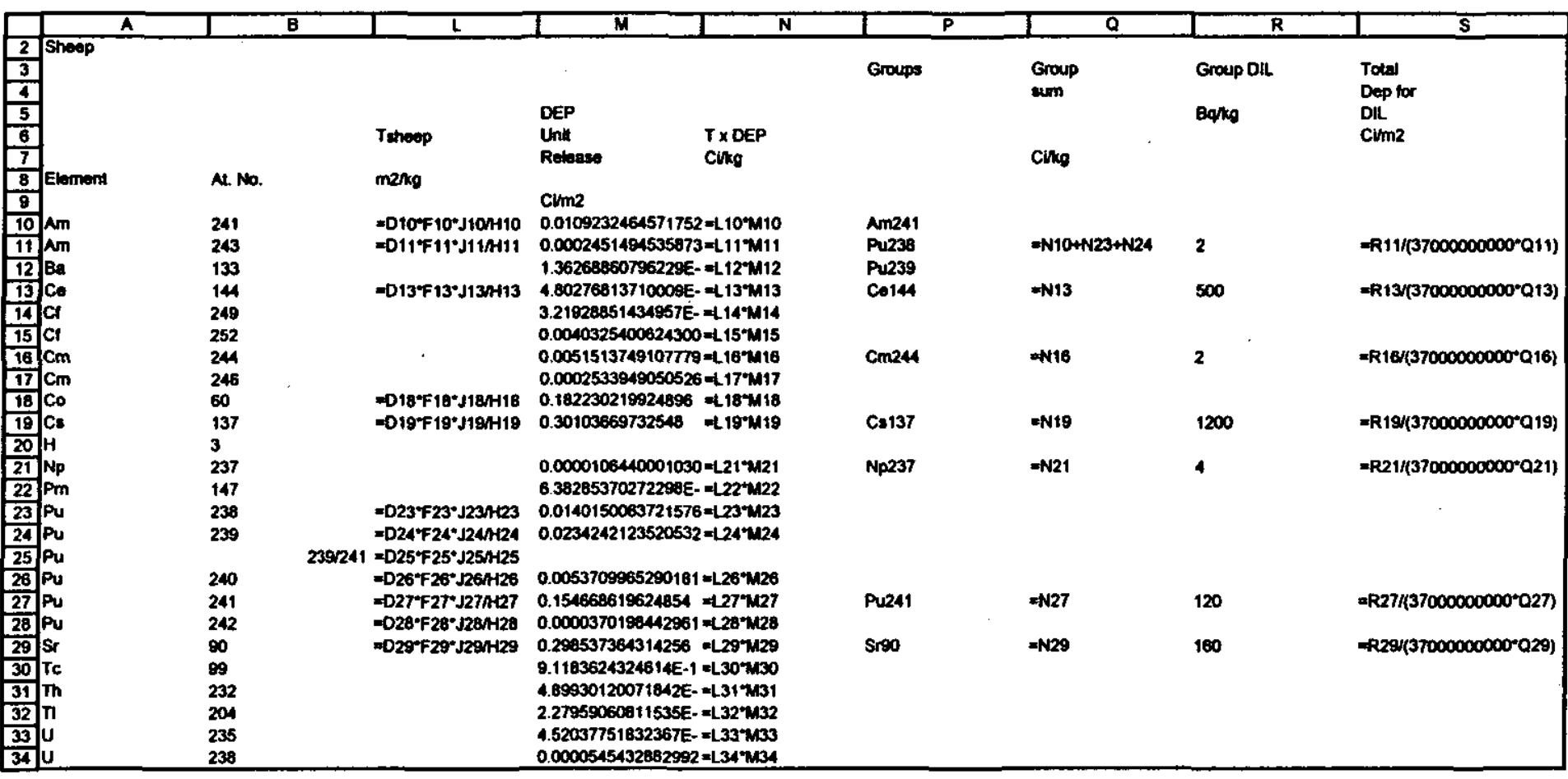




\section{Attachment G}

Contamination of lamb spreadsheet calculations

Cell references in the formulae for the overall transfer factor (Column $L$ ) were verified in S-CLC-G-00179 and are not included in this attachment. 


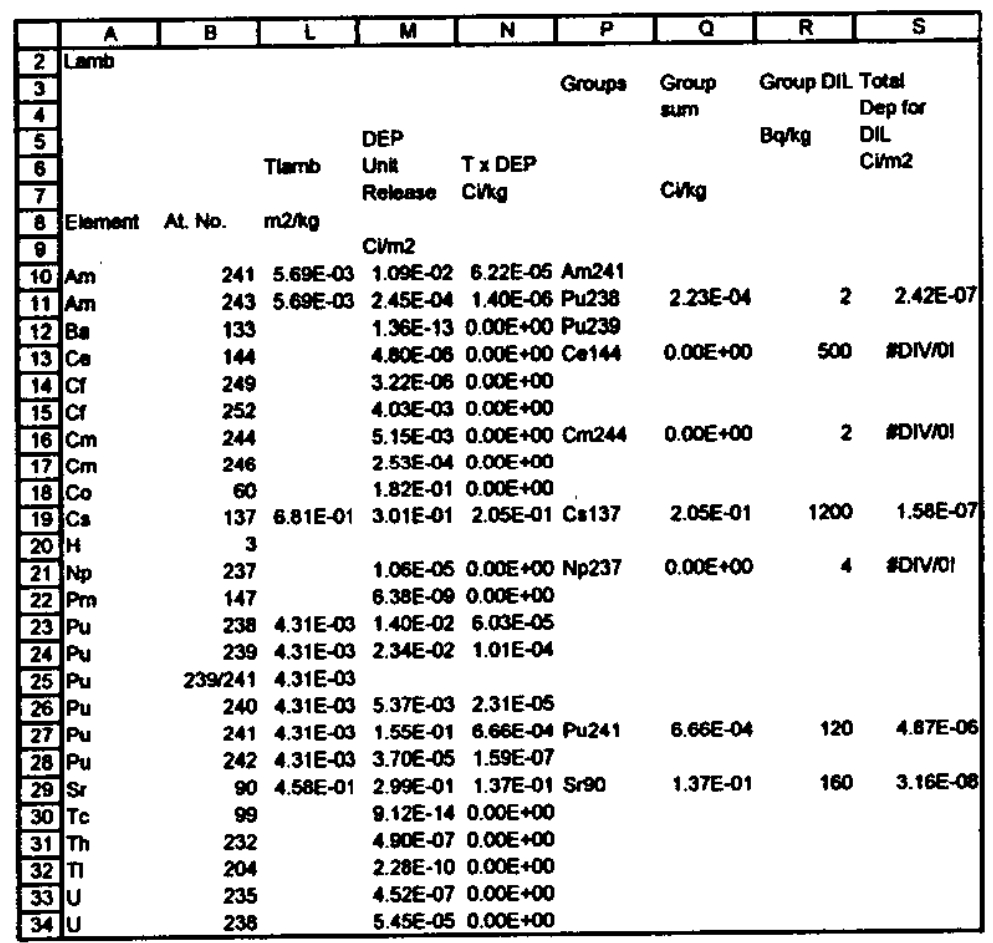




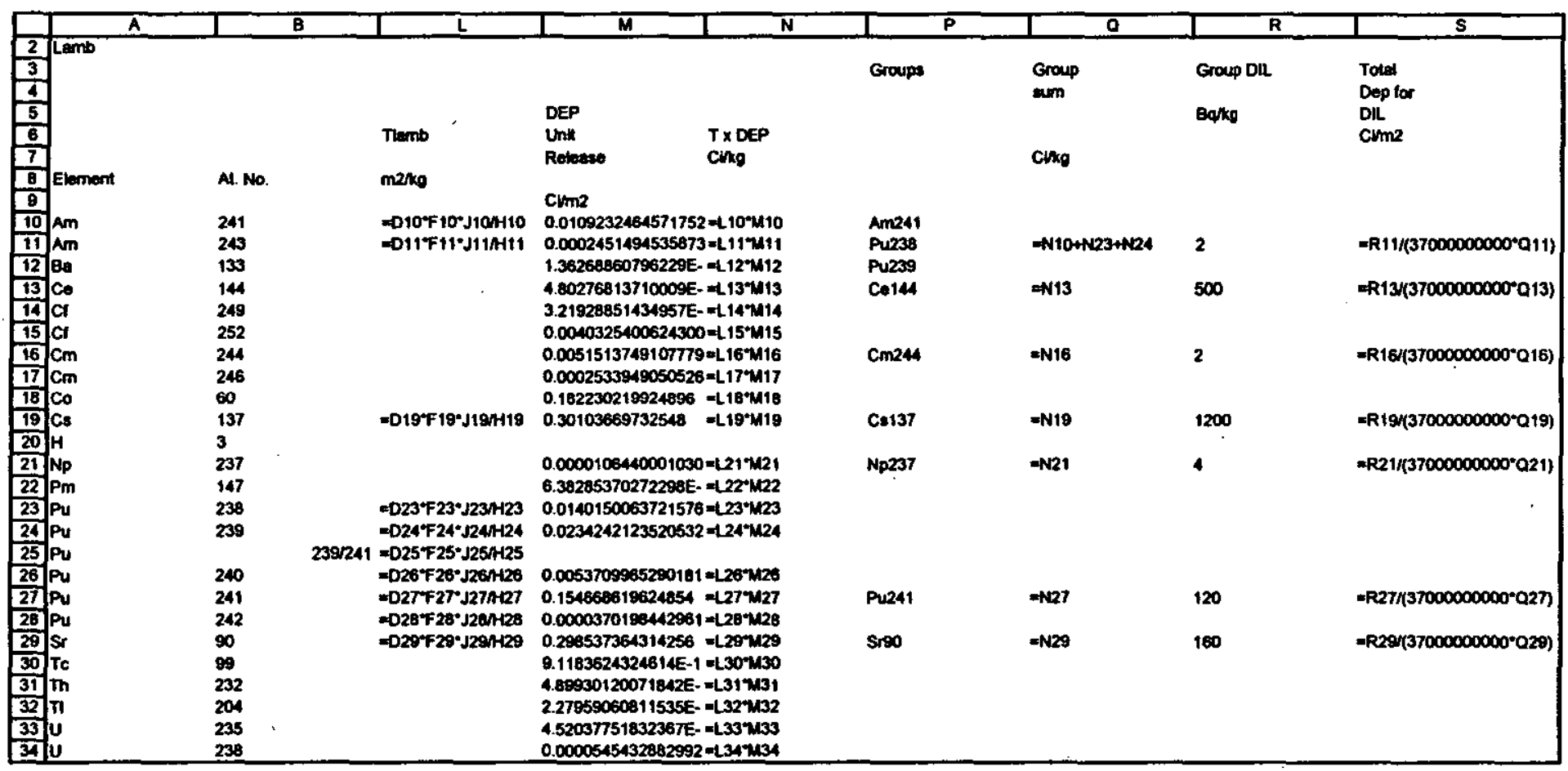




\section{Attachment $\mathrm{H}$}

Contamination of pork spreadsheet calculations

Cell references in the formulae for the overall transfer factor (Column $\mathrm{L}$ ) were verified in S-CLC-G-00179 and are not included in this attachment. 


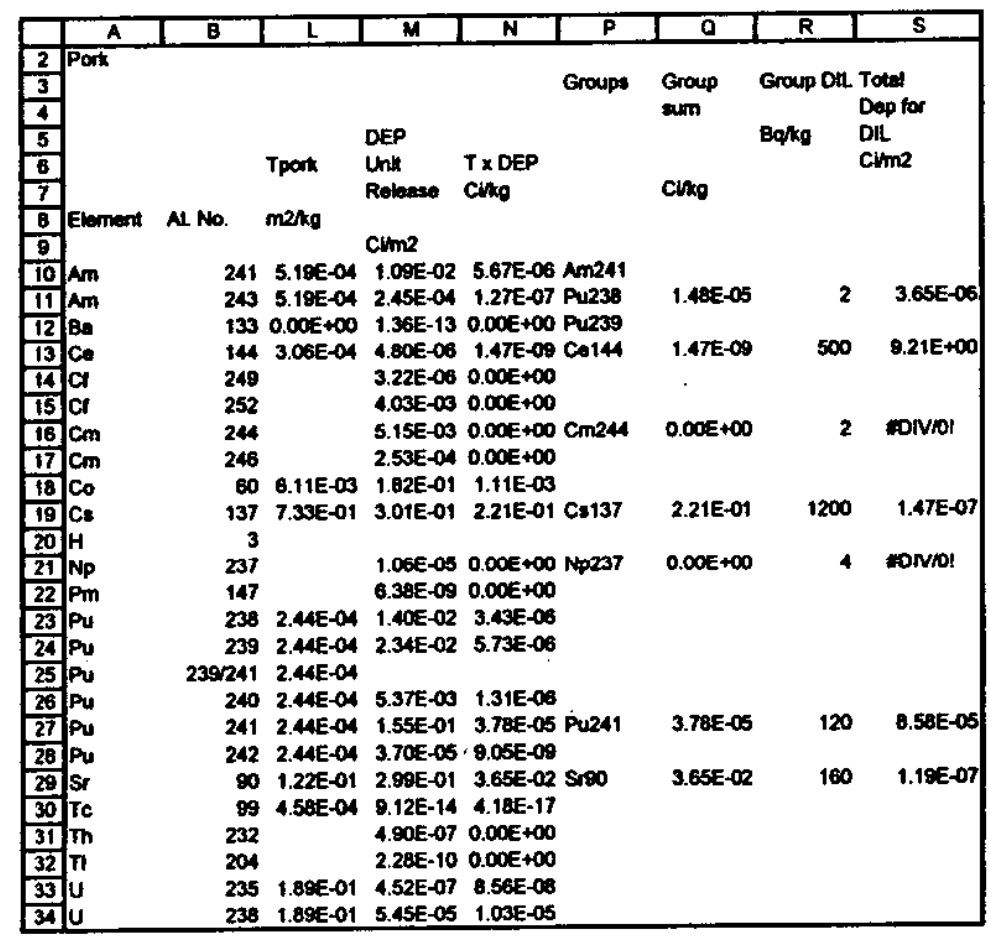




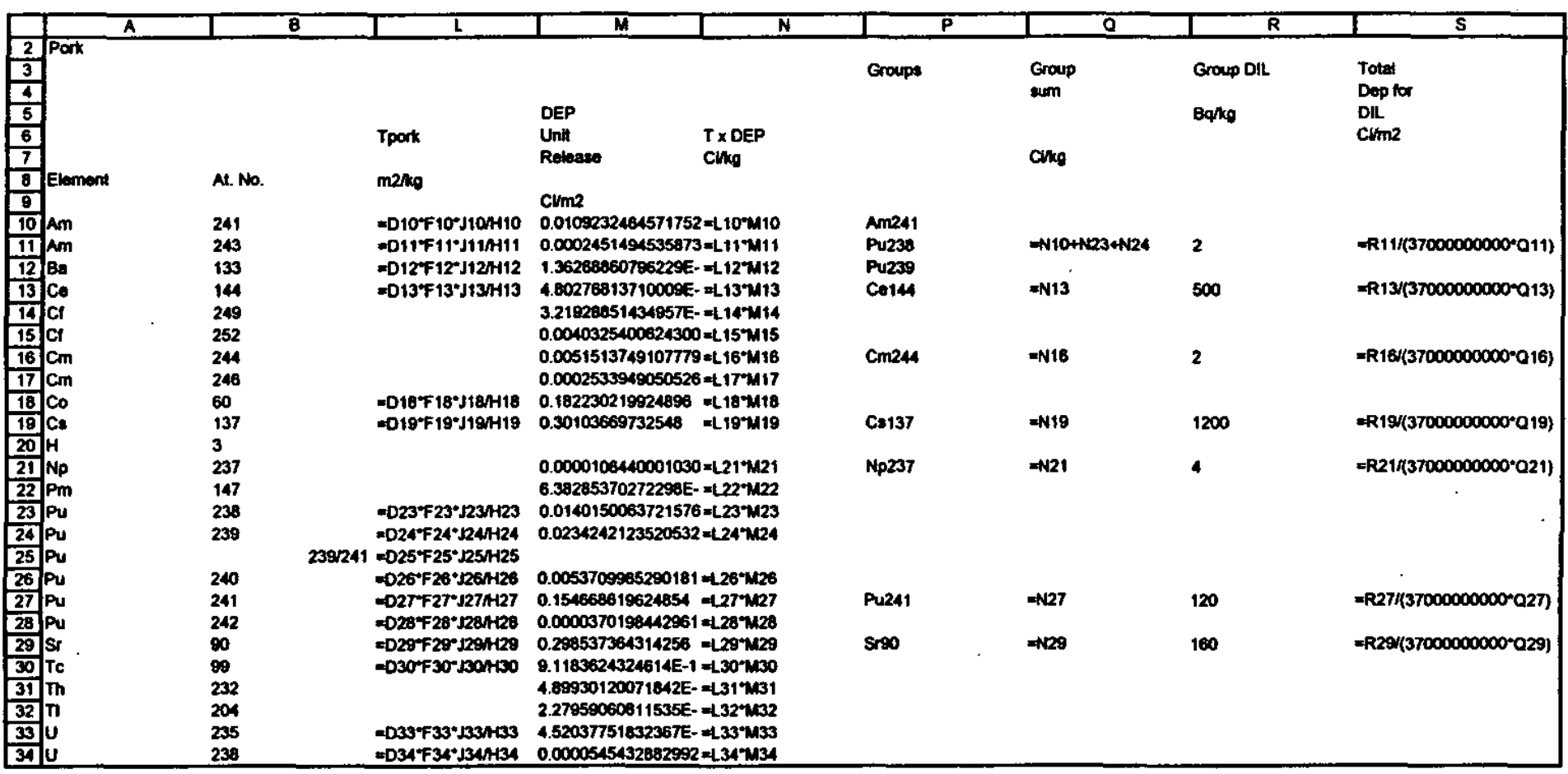




\section{Attachment I}

Contamination of poultry spreadsheet calculations

Cell references in the formulae for the overall transfer factor (Column $\mathrm{L}$ ) were verified in S-CLC-G-00179 and are not included in this attachment. 


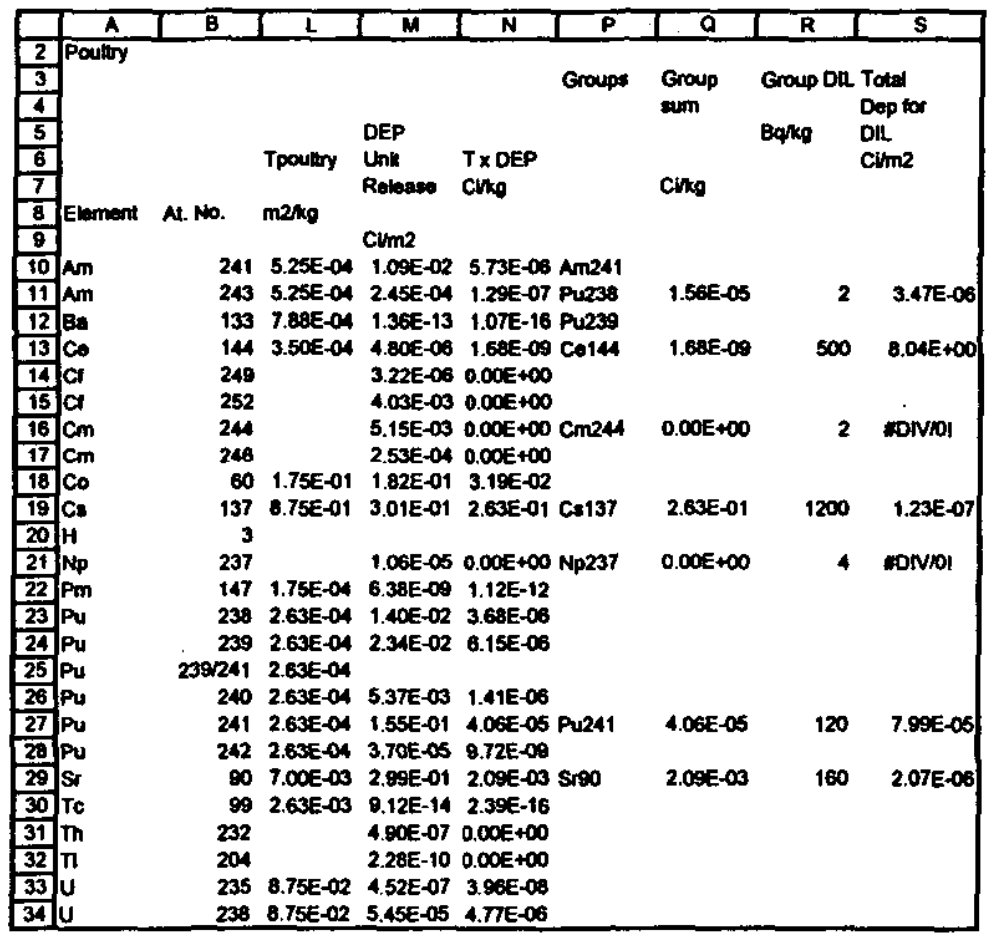




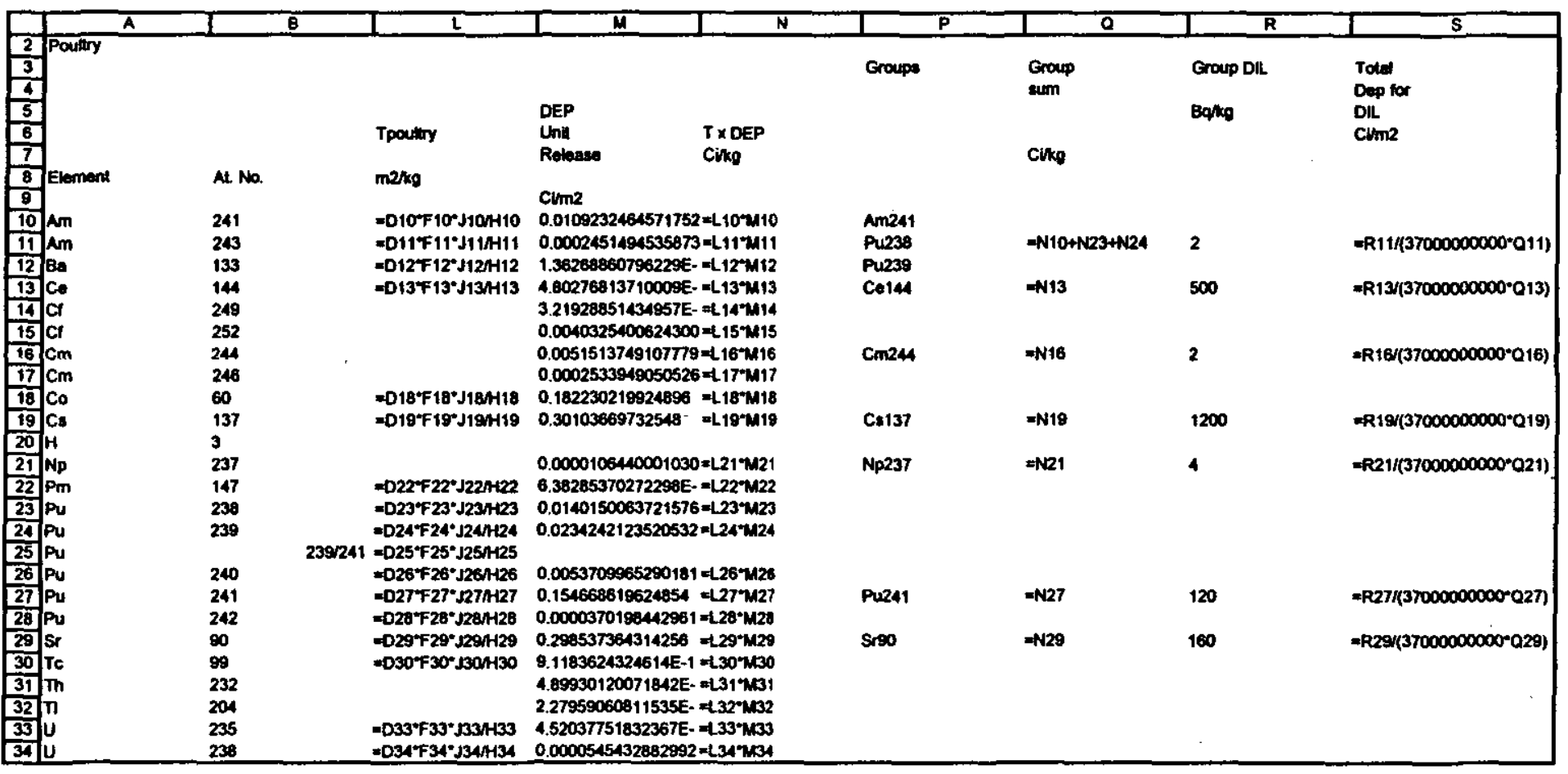




\section{Attachment J}

Contamination of fish spreadsheet calculations

Cell references in the formulae for the overall transfer factor (Column I) were verified in S-CLC-G-00179 and are not included in this attachment. 


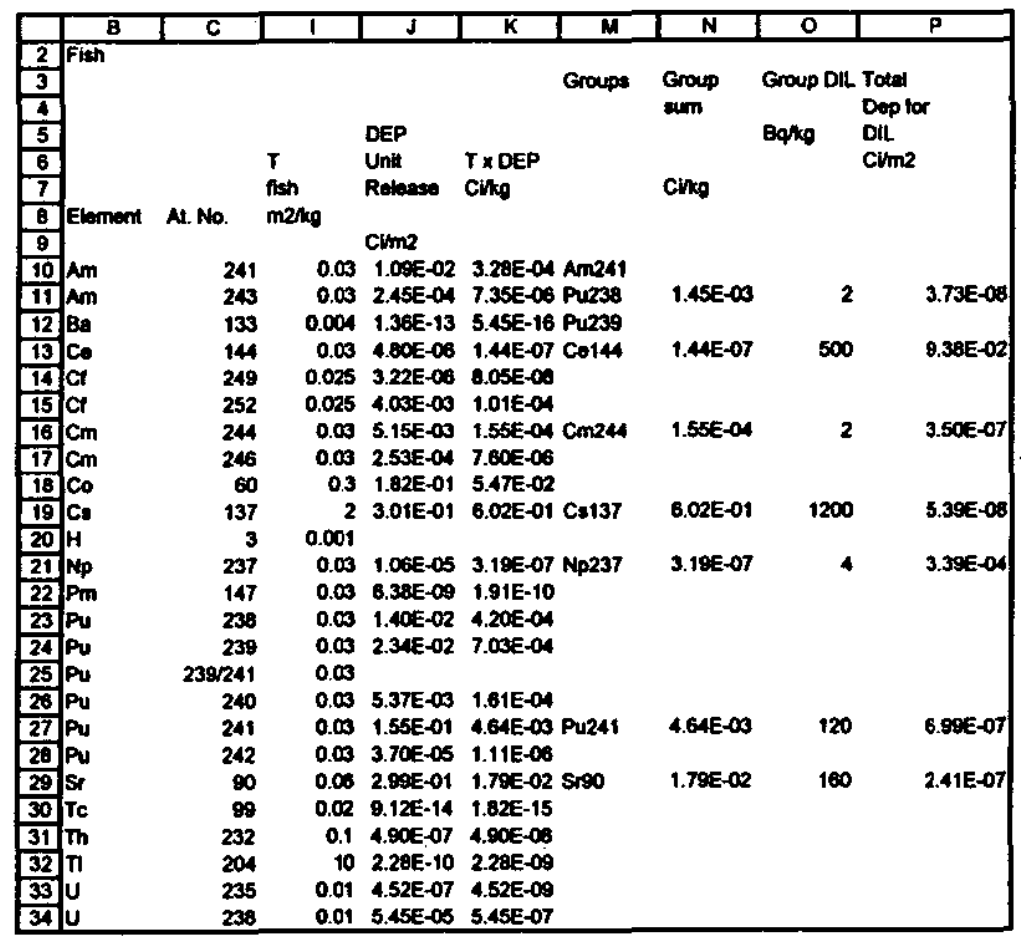




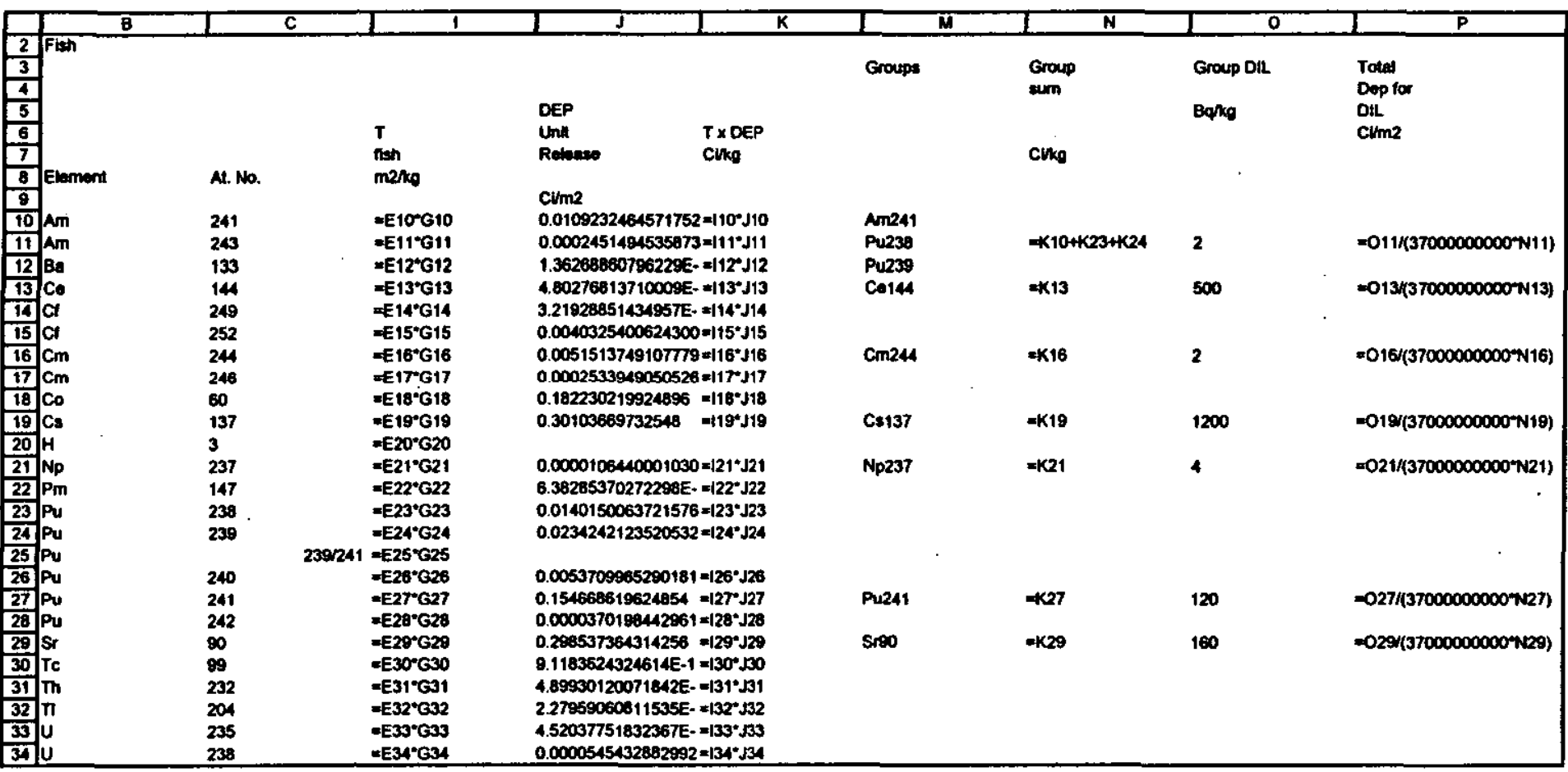




\section{Attachment K}

External contamination of produce from direct deposition spreadsheet calculations

Cell references in the formulae for the overall transfer factor (Column $\mathrm{H}$ ) were verified in S-CLC-G-00179 and are not included in this attachment. 


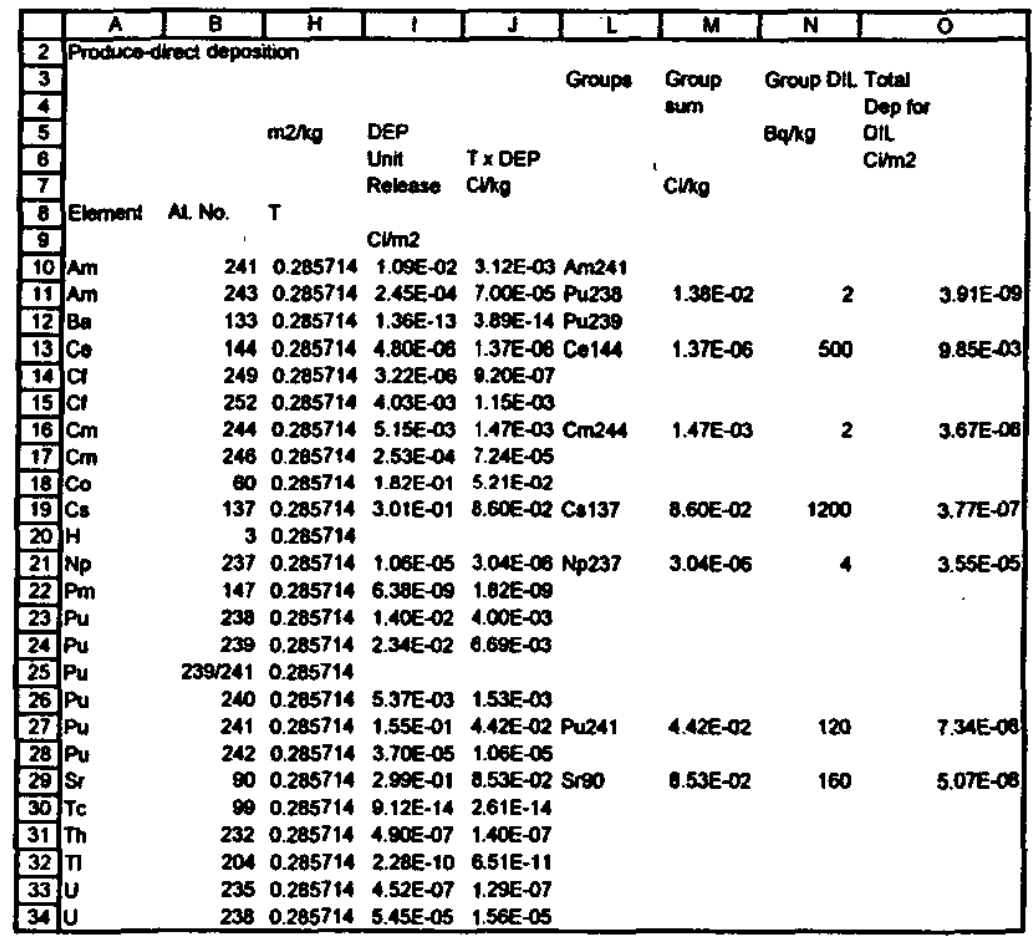




\section{Attachment L}

Internal contamination of produce from root uptake spreadsheet calculations

Cell references in the formulae for the overall transfer factor (Column $\mathrm{H}$ ) were verified in S-CLC-G-00179 and are not included in this attachment. 


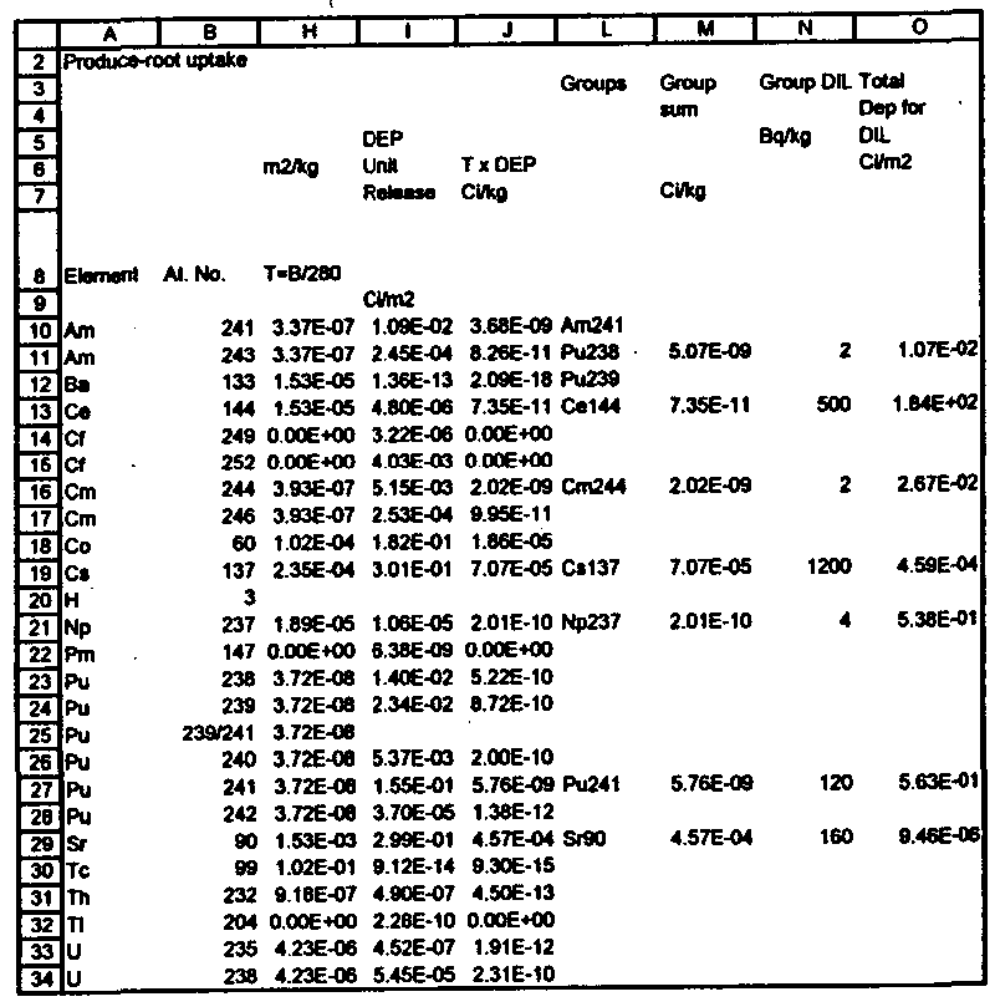




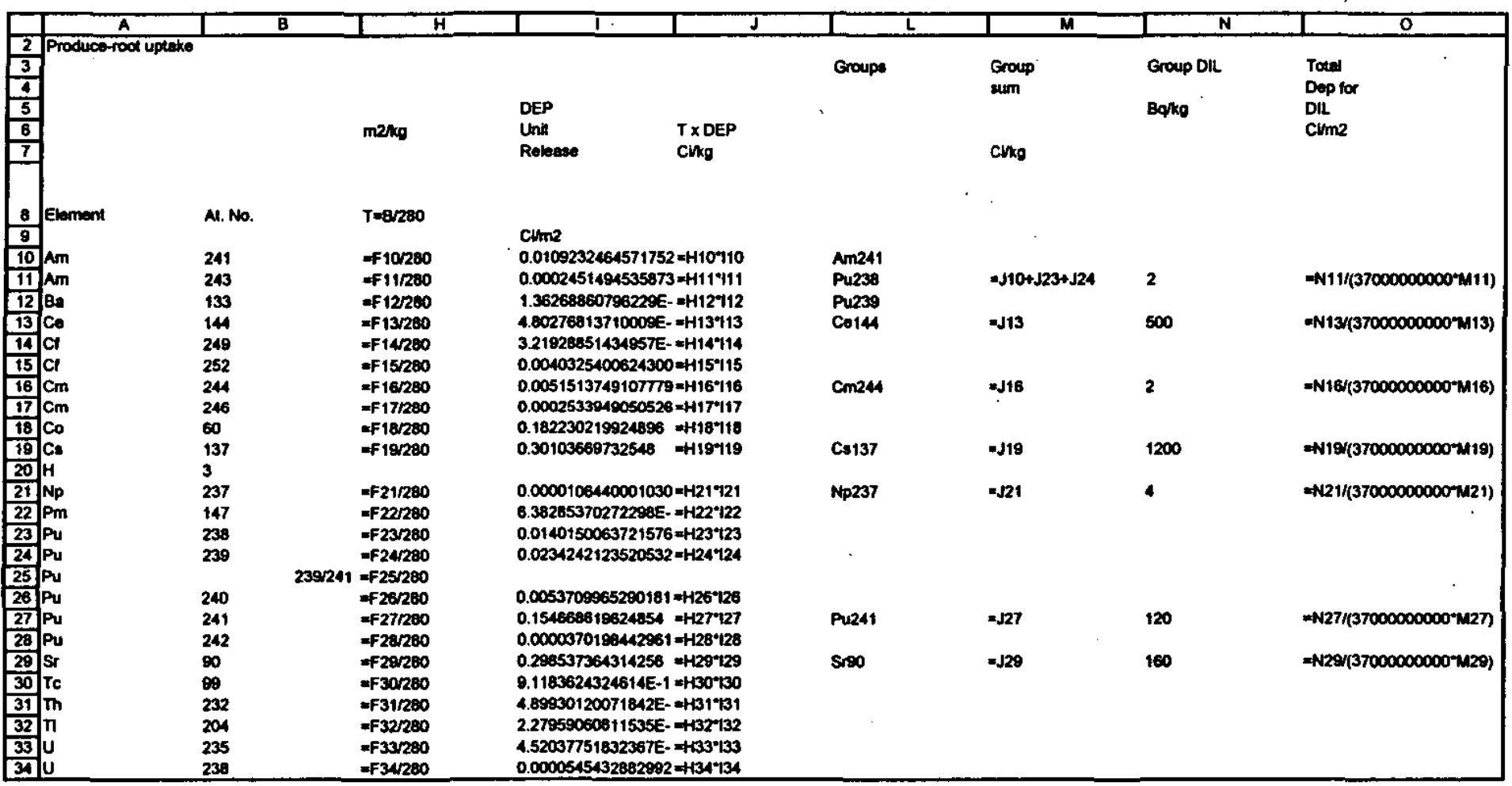




\section{Attachment M}

External contamination of leafy vegetables during harvest spreadsheet calculations

Cell references in the formulae for the overall transfer factor $($ Column $\mathrm{H})$ were verified in S-CLC-G-00179 and are not included in this attachment. 


\begin{tabular}{|c|c|c|c|c|c|c|c|c|c|}
\hline & A & 日 & H & $T$ & $J$ & $\bar{L}$ & $M$ & $\overline{\mathbf{N}}$ & 0 \\
\hline 2 & Produce- & ioll acthesion & & & & & & & \\
\hline 4 & & & & & & Groupsis & sump & Goup oit & Dep for \\
\hline 5 & & & & DEP & & & & Bqug & \\
\hline 6 & & & & Unit & $T \times$ DEP & & & & $\mathrm{Clma2}$ \\
\hline 7 & Element & At No & $T=D$ Epen & Rolease & Cikg & & Cikg & & \\
\hline 9 & & & & Cim2 & & & & & \\
\hline 10 & Am & 241 & $5.10204 E-06$ & $1.09 E-02$ & $5.57 E-00$ & Am241 & & & \\
\hline 111 & Am & 243 & $5.10204 E-06$ & $2.45 E-0.4$ & $1.25 E-09$ & Pu238 & 2.47E-07 & 2 & 2.18E-04 \\
\hline 12 & Ba & 133 & $5.10204 E-06$ & 1.36E-13 & B.95E-19 & Pu239 & & & \\
\hline 13 & co & 144 & $5.10204 \mathrm{E}-06$ & 4.80E-06 & $2.45 E-11$ & Cols4 & 2.45E-11 & 500 & $5.51 E+02$ \\
\hline 14 & ca & 249 & 5. 1020 AnE-06 & 3.22E-06 & $1.64 E-11$ & & & & \\
\hline 15 & & 252 & 5.10204E-06 & 4.03E-03 & $2.06 E-08$ & & & & \\
\hline 16 & $\mathrm{Cm}$ & 244 & 5.10204E-06 & $5.15 E-03$ & $2.63 E-06$ & $\mathrm{Cm} 244$ & 2.63E-08 & 2 & $2.06 E-\infty 3$ \\
\hline 17 & $\mathrm{Cm}$ & 246 & $5.10204 E-08$ & 2.53E-04 & $1.29 E-\infty$ & & & & \\
\hline 18 & & $\begin{array}{r}60 \\
137\end{array}$ & 5.10204E-06 & 1.82E-01 & $9.30 E-07$ & & & & \\
\hline$\frac{19}{20}$ & & $\begin{array}{r}137 \\
3\end{array}$ & $\begin{array}{l}5.10204 E-06 \\
5.10204 E-06\end{array}$ & $3.01 E-01$ & $1.54 E-06$ & $\cos 137$ & $1.54 E-08$ & 1200 & $2.111-02$ \\
\hline 21 & & 237 & $5.10204 E-06$ & $1.06 E-05$ & $5.43 E-11$ & Np237 & $5.43 E-11$ & 4 & $1.99 E+00$ \\
\hline $22 P$ & Pm & 147 & $5.10204 E-06$ & 6.38E-09 & $3.26 E-14$ & & & & \\
\hline $23 \mathrm{~F}$ & Pu & 238 & 5.10204E-06 & $1.40<-02$ & $7.15 E-08$ & & & & \\
\hline $24 \mathrm{p}$ & Py & 239 & $5.10204 E-06$ & $2.34 E-02$ & $1.20 E-07$ & & & & \\
\hline$\left.25\right|_{p} ^{p}$ & Pu & 239241 & $5.10204 E-08$ & & , TMEת & & & & \\
\hline 27 & & 241 & $5.10204 E-08$ & 1.55E-01 & 7.ase-07 & Pu241 & $7.896-07$ & 120 & $4.11 E-\infty 3$ \\
\hline 28 & Pu & 242 & 5.10204E-08 & 3.70E-CS & 1.09E-10 & & & & \\
\hline$\frac{29}{30}$ & & 90 & $5.10204 E-08$ & $2.99 E-01$ & $\begin{array}{l}1.52 E-06 \\
65 E-10\end{array}$ & sreo & $1.52 \mathrm{E}-06$ & 100 & $2.84 E-03$ \\
\hline 31 & $m$ & 232 & 5.1020AE-06 & $\begin{array}{l}8.90 E=-07 \\
4.907\end{array}$ & $2.50 E-12$ & & & & \\
\hline 32 & & 204 & $5.10204 E-06$ & 2.28E-10 & $1.16 E-15$ & & & & \\
\hline 3330 & & 236 & $5.10204 E-06$ & 4.52E-07 & $2.31 E-12$ & & & & \\
\hline 34 & & $230^{2}-1$ & $5.10204 E-06$ & $5.45 E-\infty 6$ & $2.78 E-10$ & & & & \\
\hline
\end{tabular}




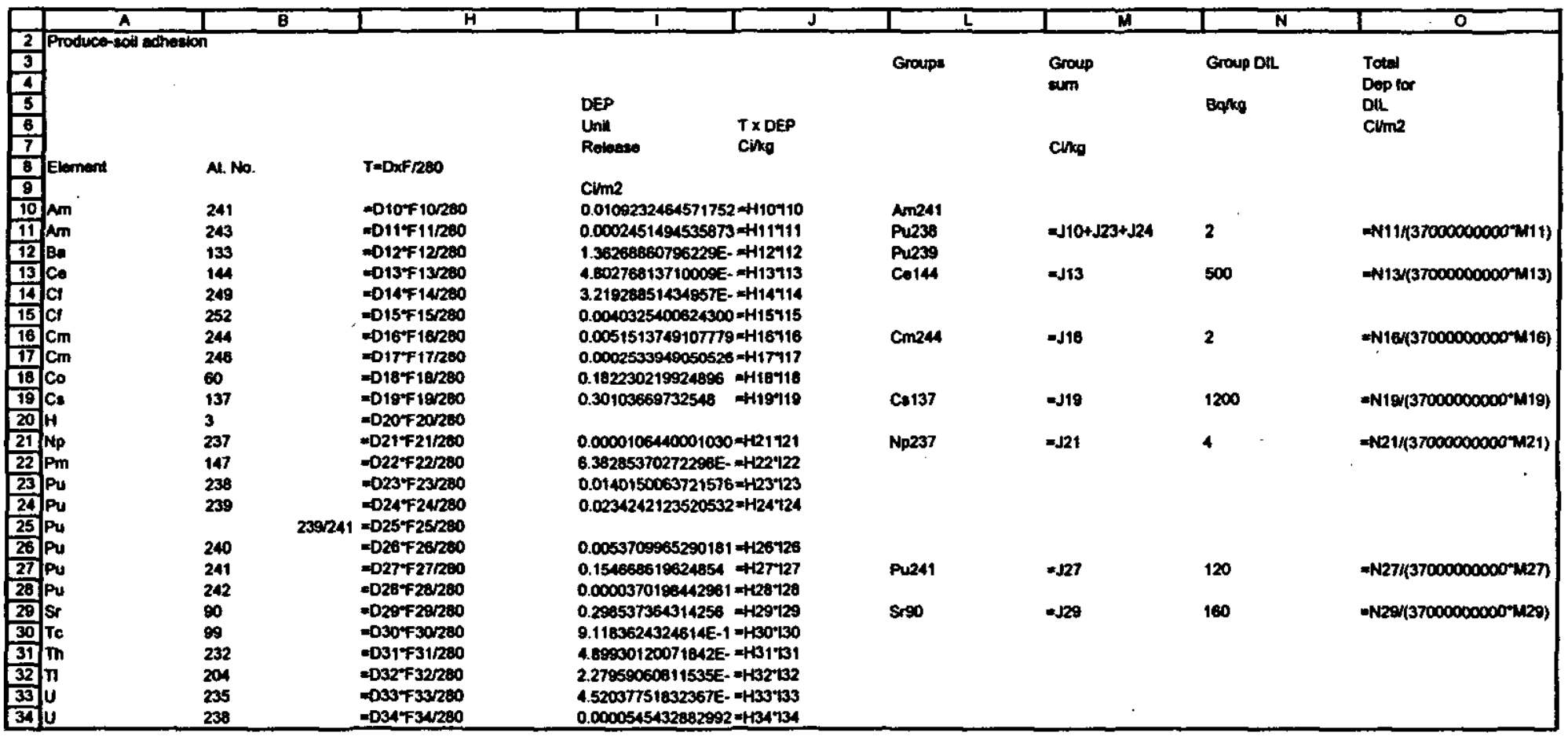




\section{Attachment $\mathbf{N}$}

External contamination of grain spreadsheet calculations

Cell references in the formulae for the overall transfer factor (Column $\mathrm{H}$ ) were verified in S-CLC-G-00179 and are not included in this attachment. 


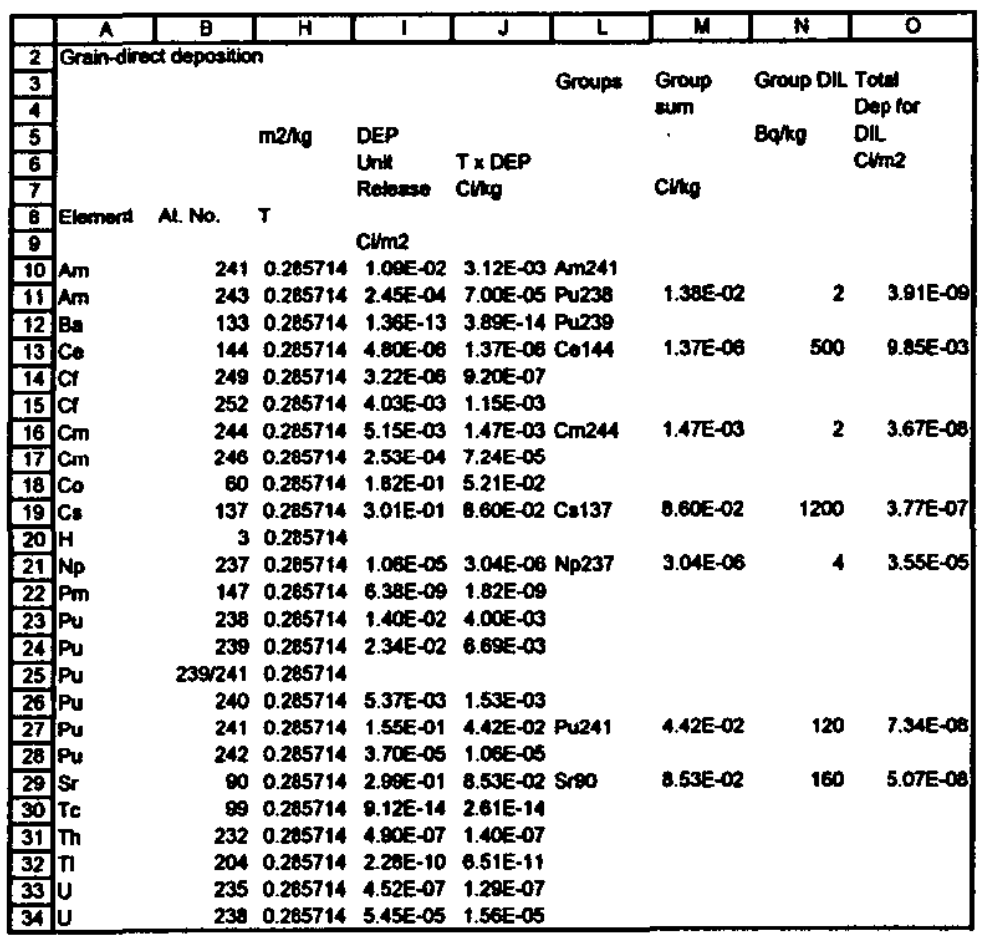




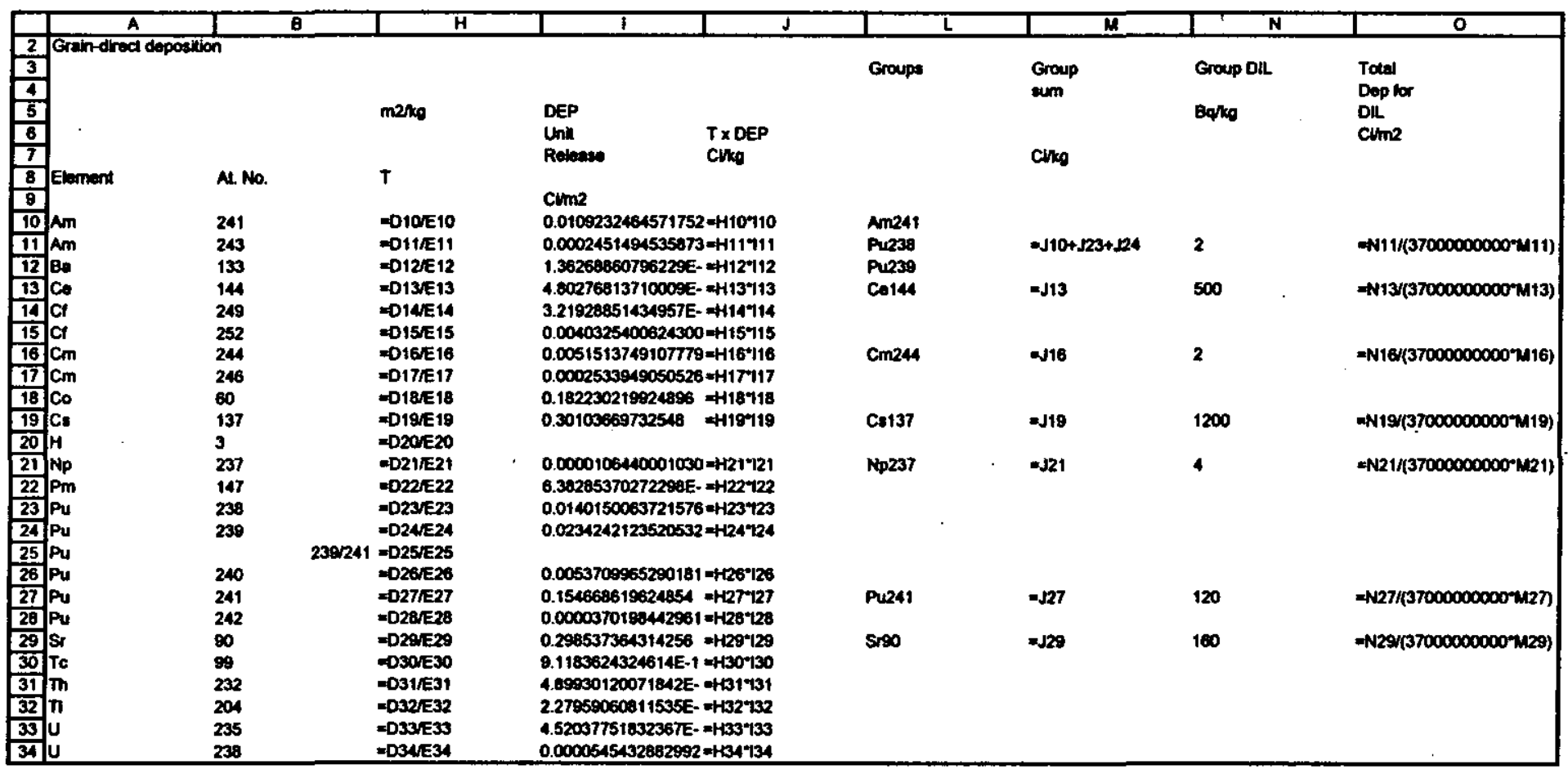




\section{Attachment $\mathrm{O}$}

Internal contamination of grain by root uptake spreadsheet calculations

Cell references in the formulae for the overall transfer factor (Column $\mathrm{H}$ ) were verified in S-CLC-G-00179 and are not included in this attachment. 


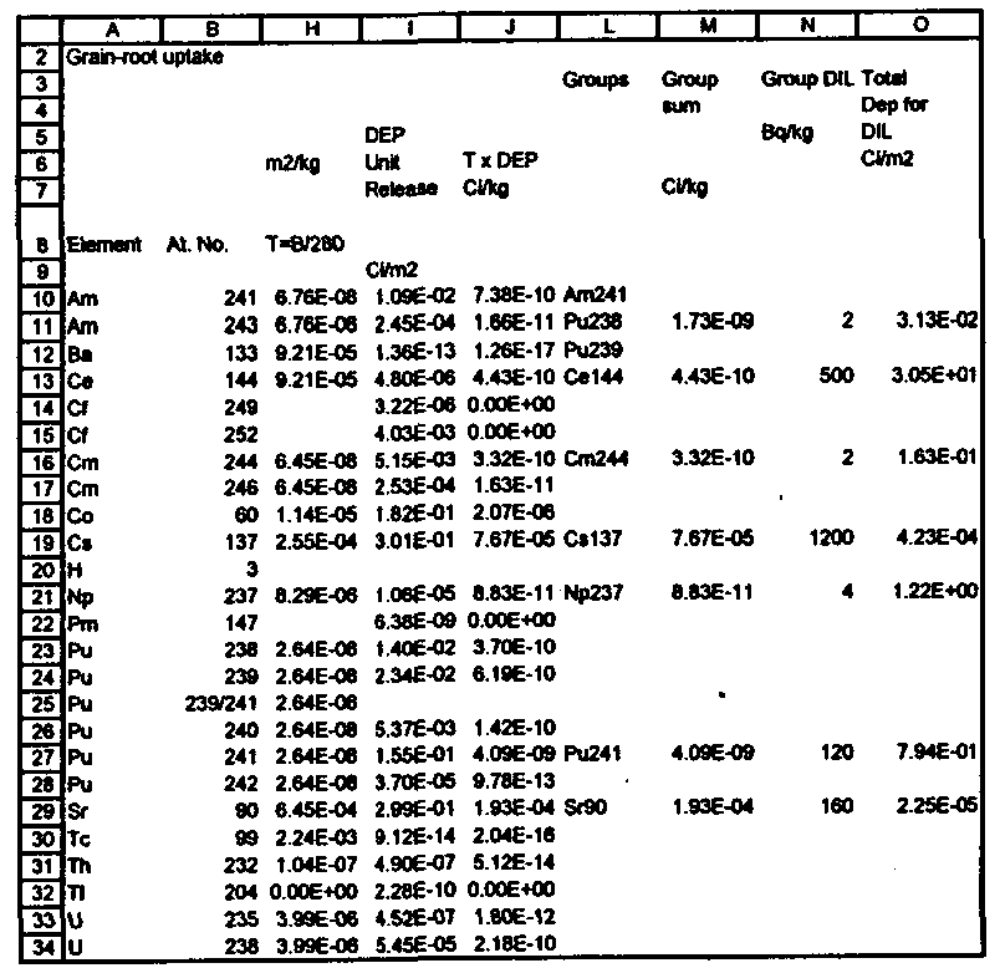




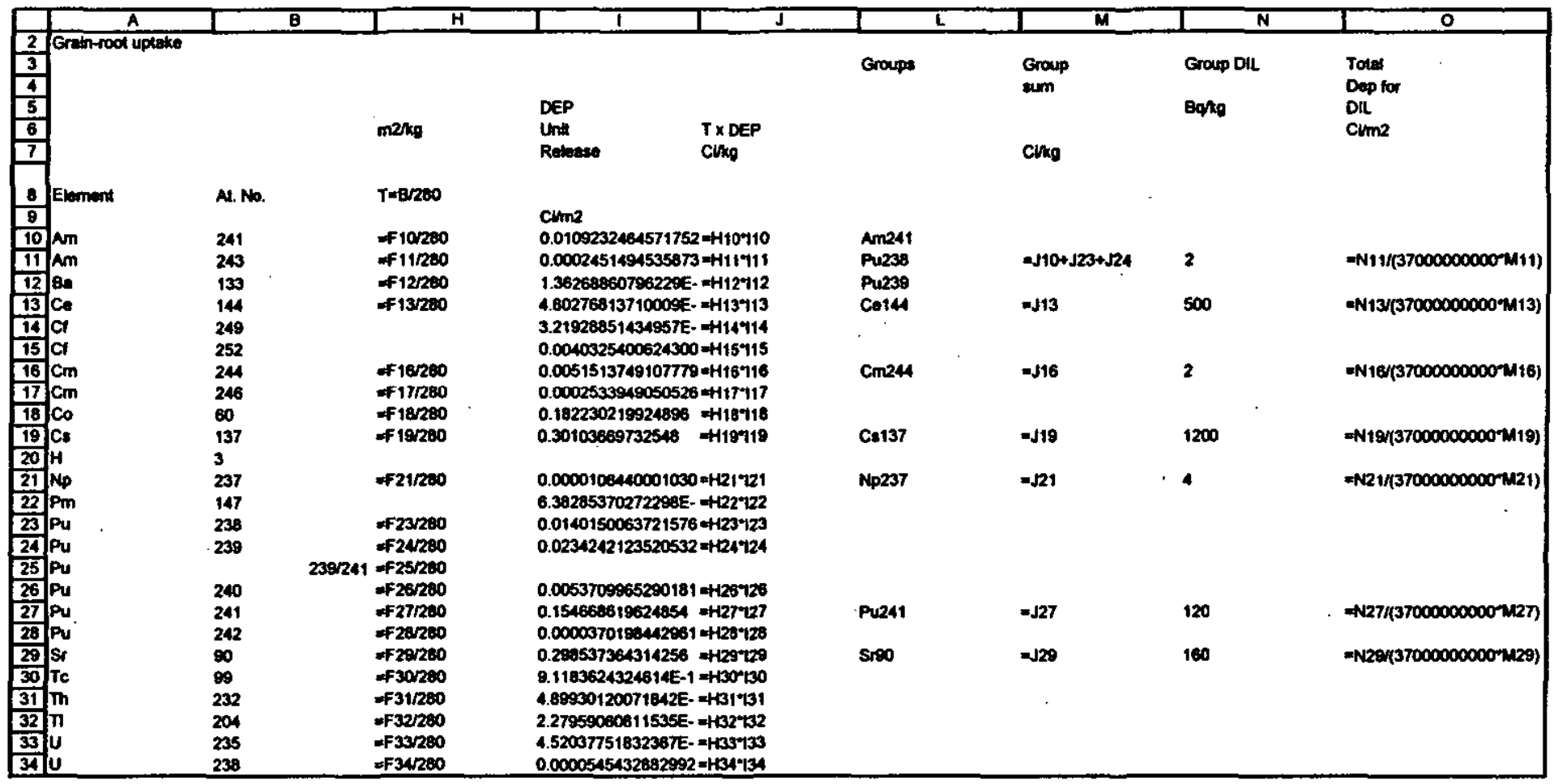




\section{Attachment $\mathbf{P}$}

External contamination of grain during harvest spreadsheet calculations

Cell references in the formulae for the overall transfer factor (Column $\mathrm{H}$ ) were verified in S-CLC-G-00179 and are not included in this attachment. 


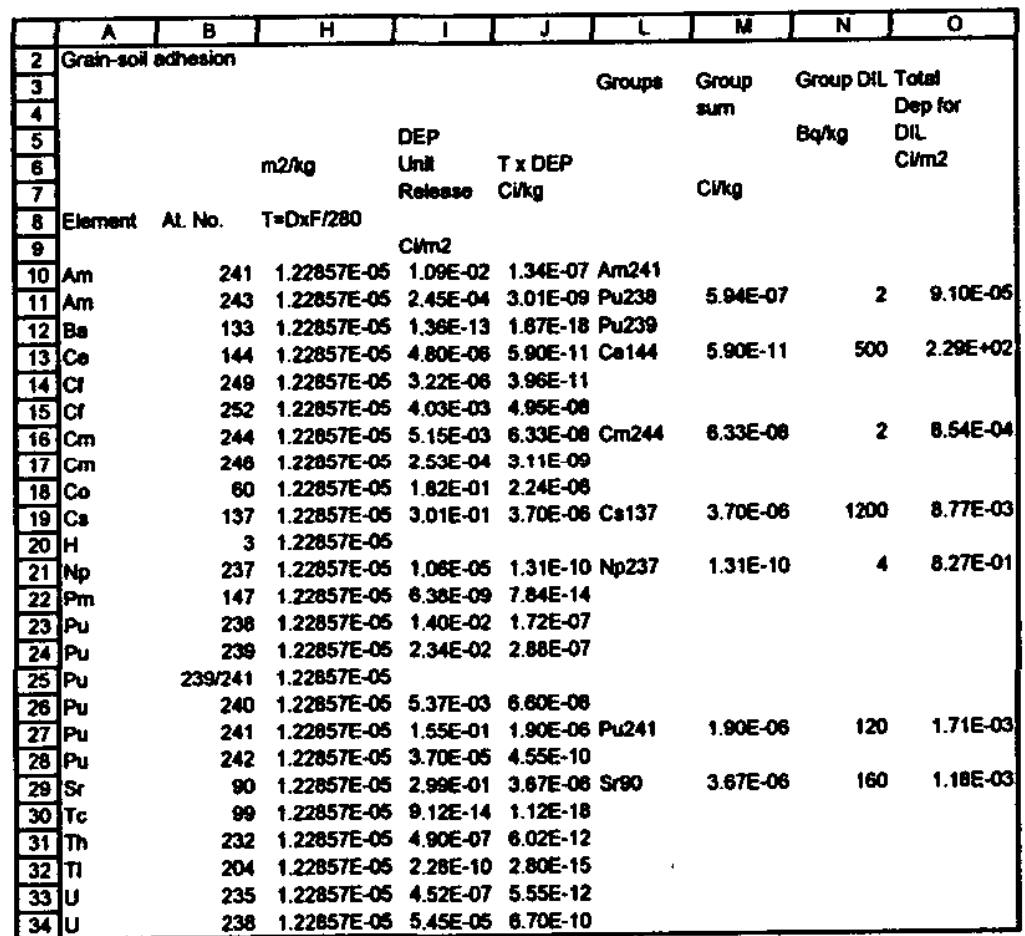




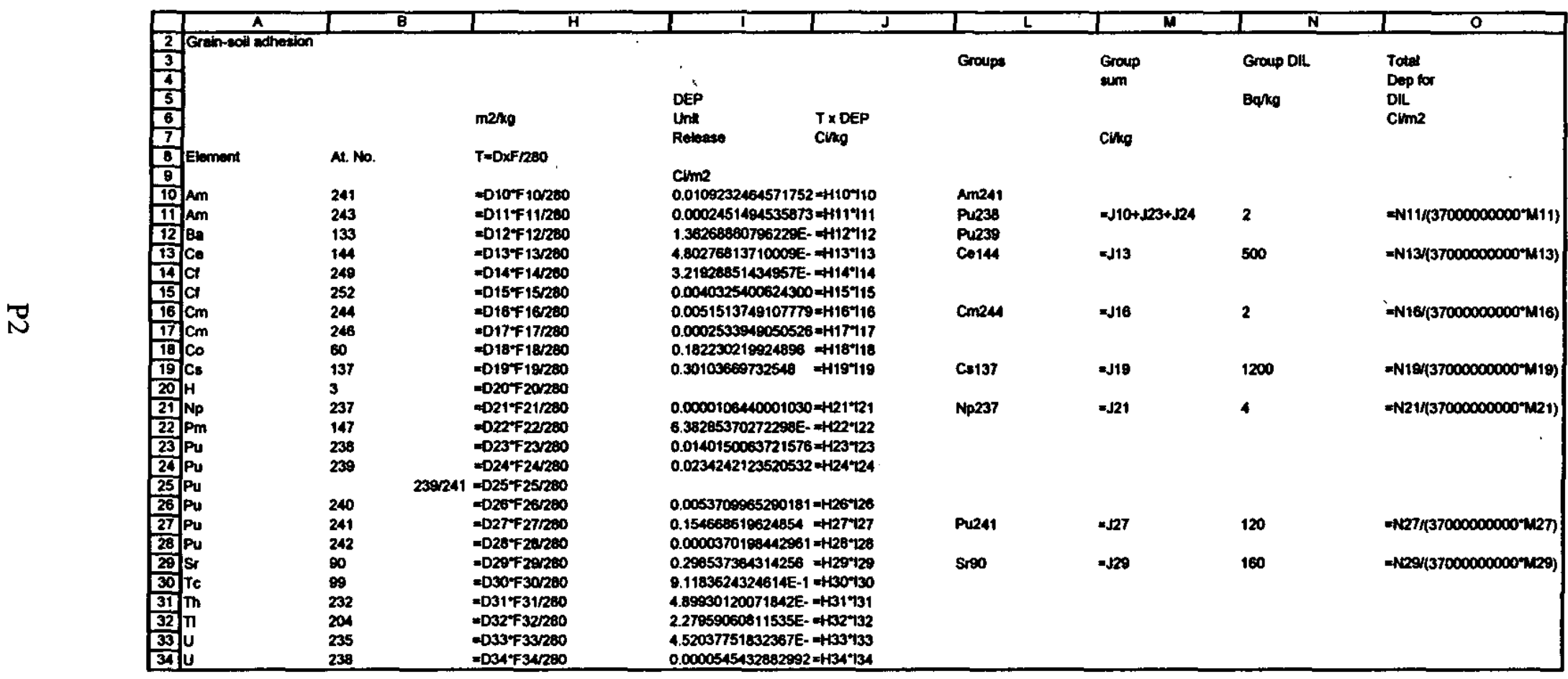




\section{Attachment Q}

Contamination of beverages spreadsheet calculations 


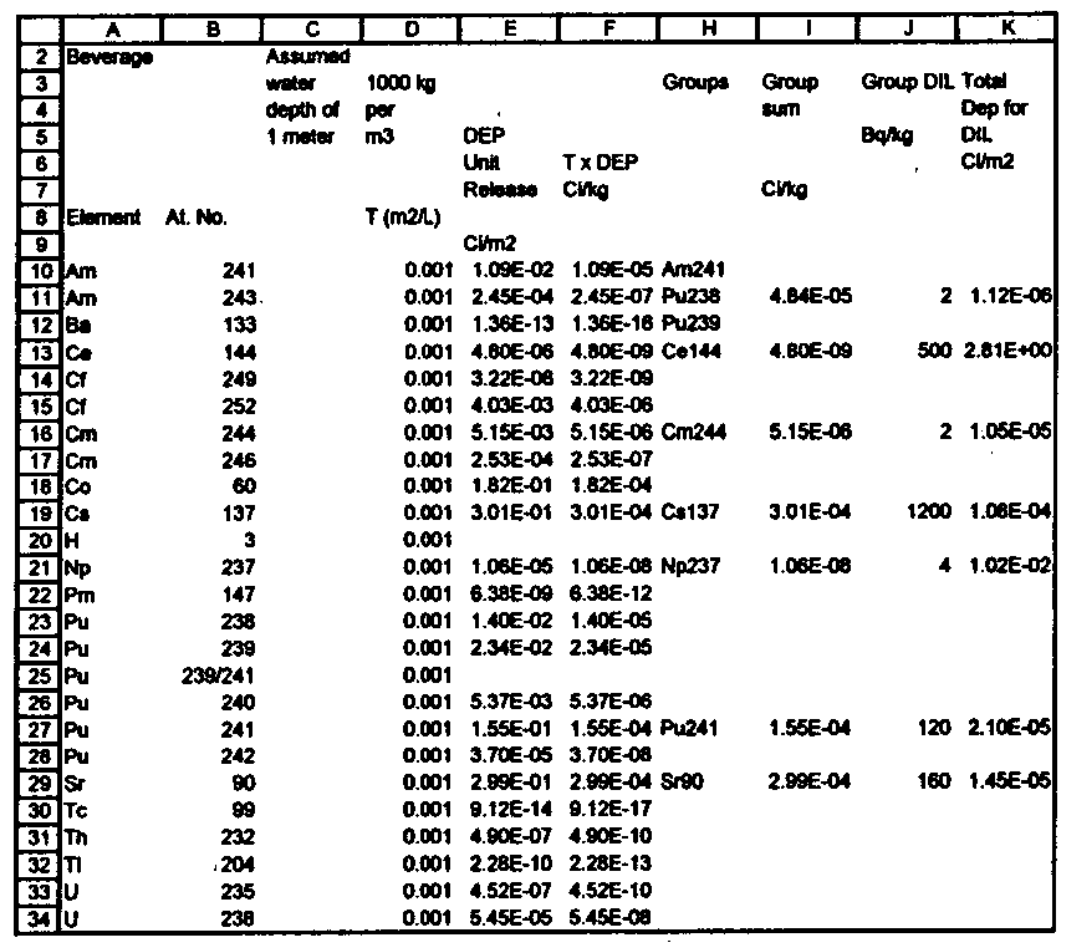




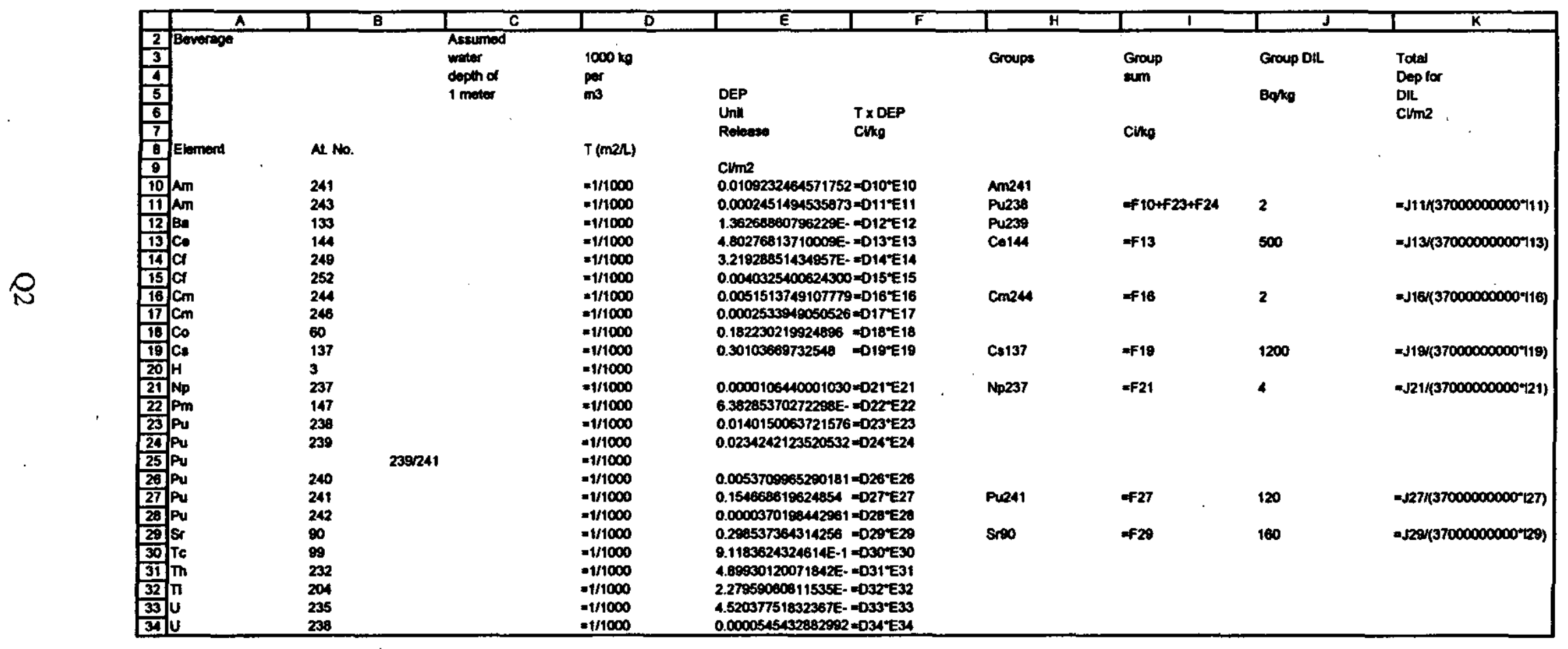




\section{Attachment $\mathbf{R}$}

HOTSPOT results - average meteorology, $0.1 \mathrm{~cm} \mathrm{~s}^{-1}$ 

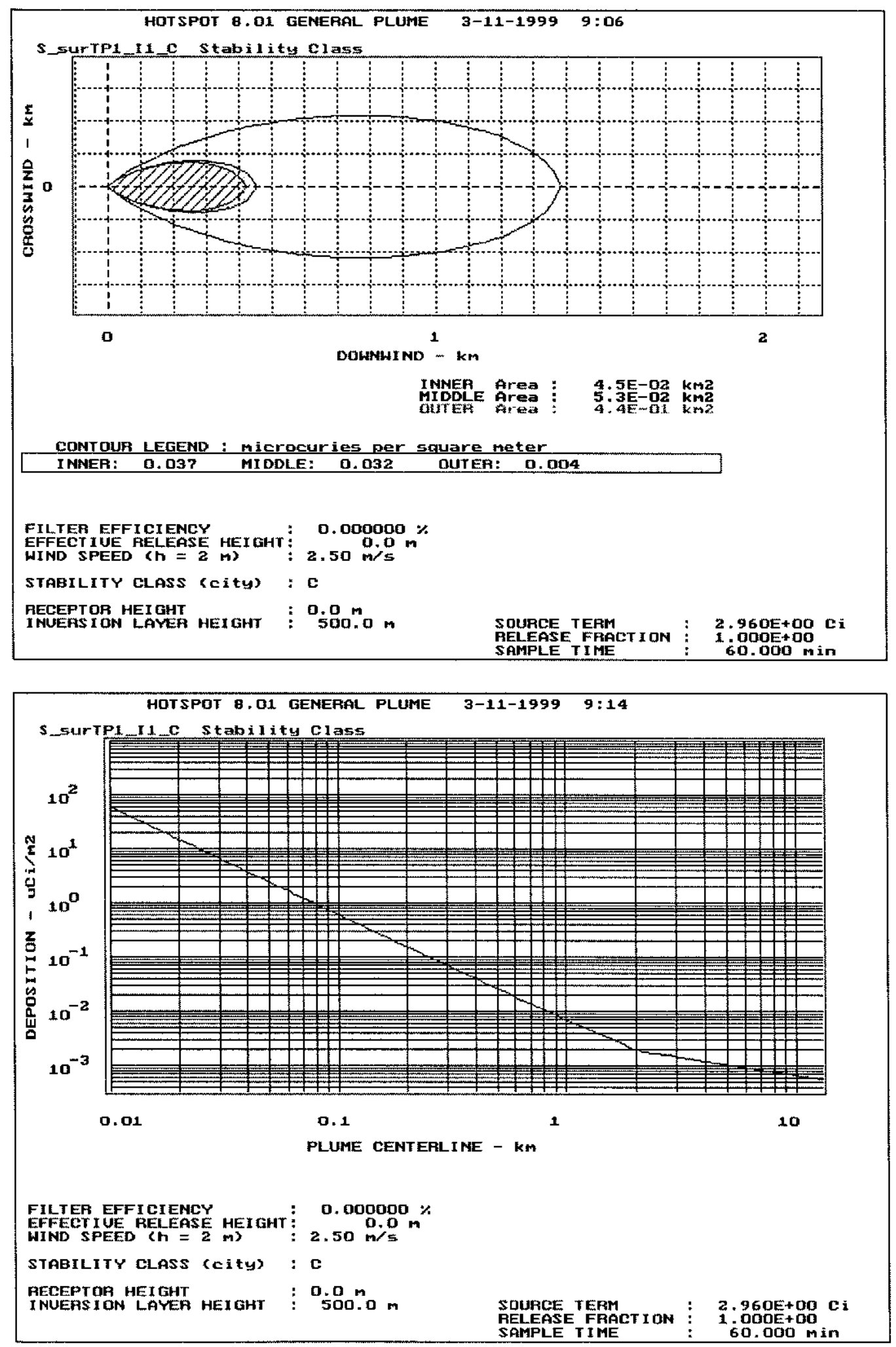

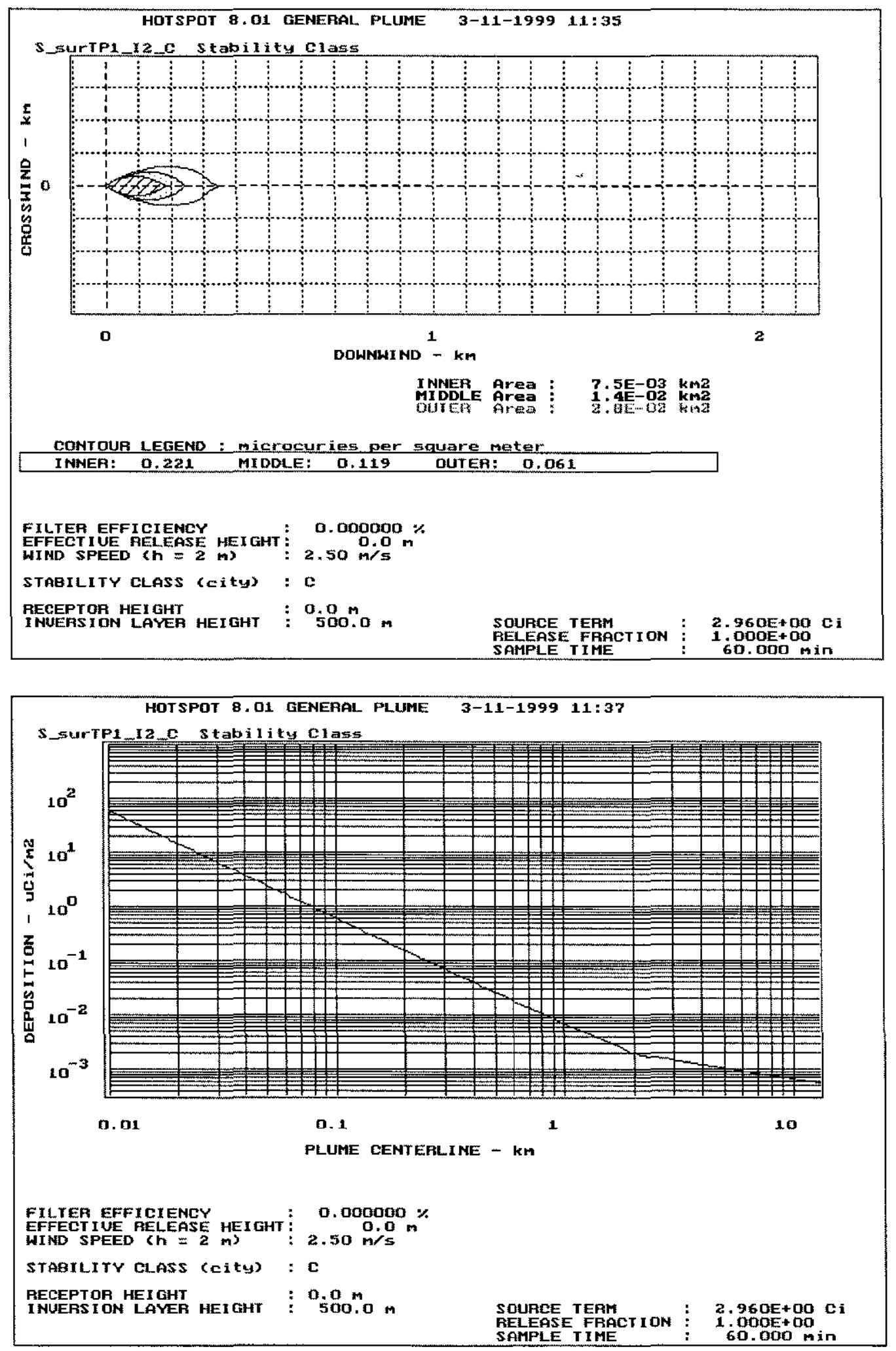

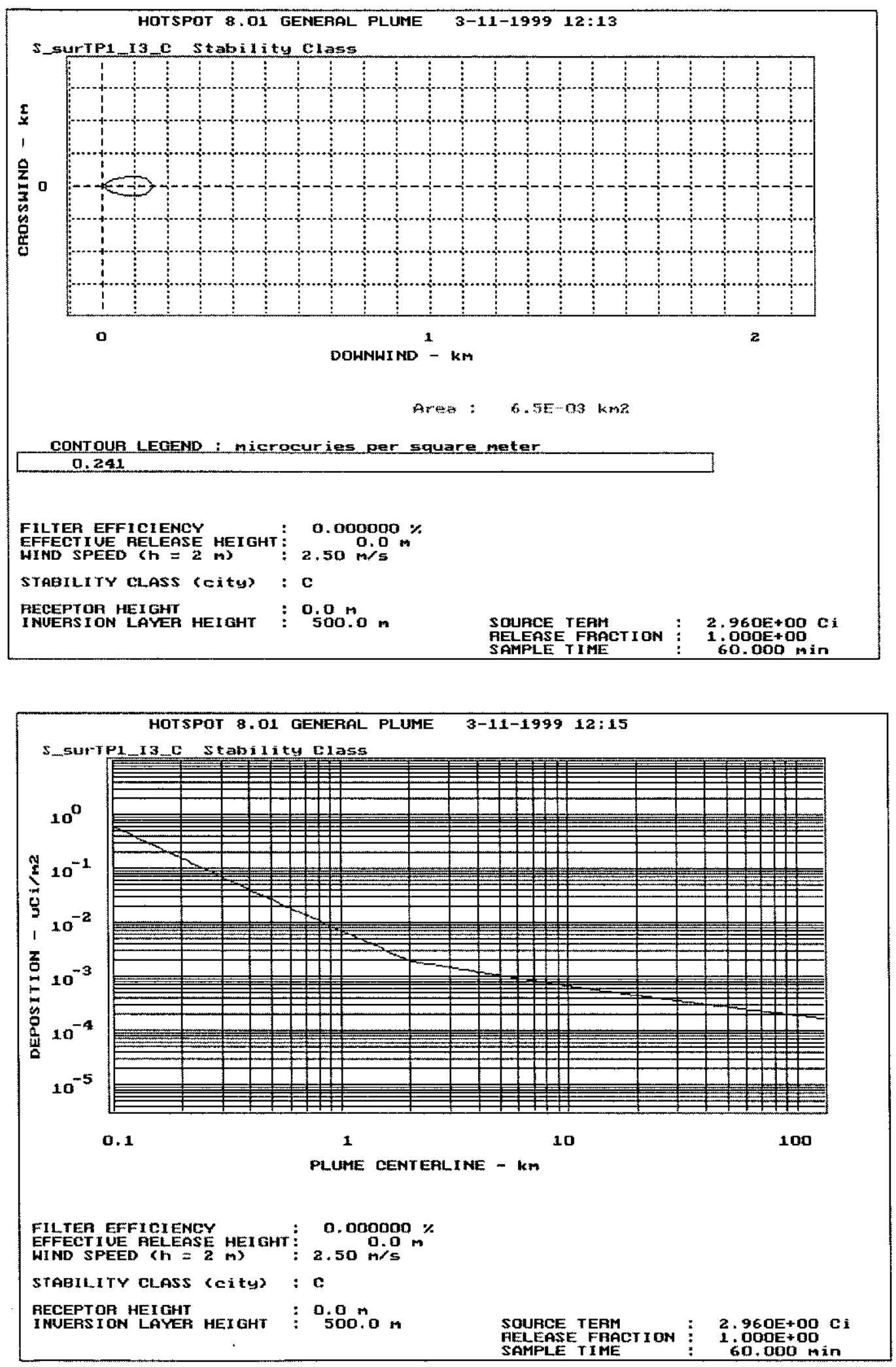

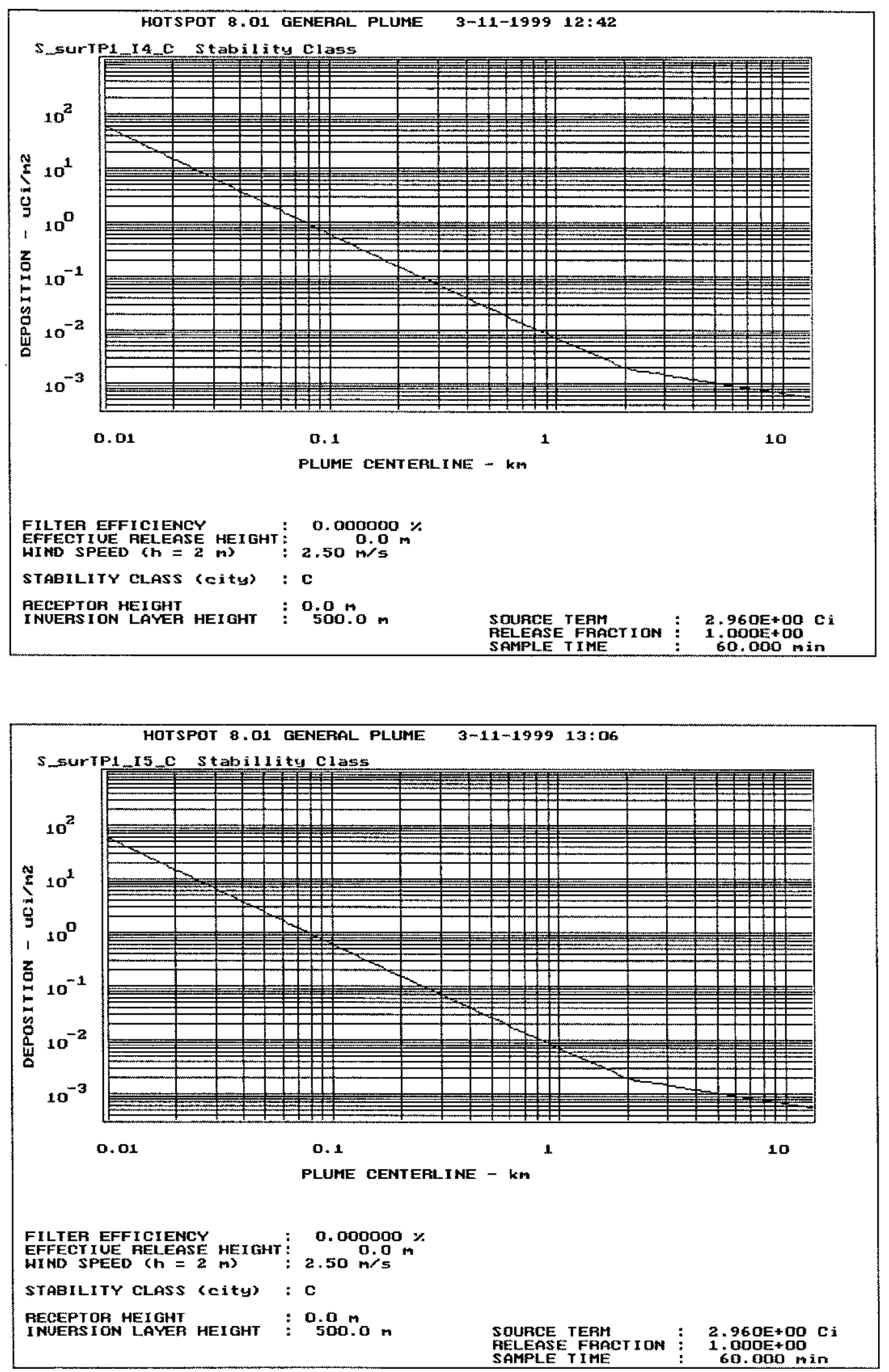


\section{Attachment S}

HOTSPOT results - average meteorology, $1.0 \mathrm{~cm} \mathrm{~s}^{-1}$ 

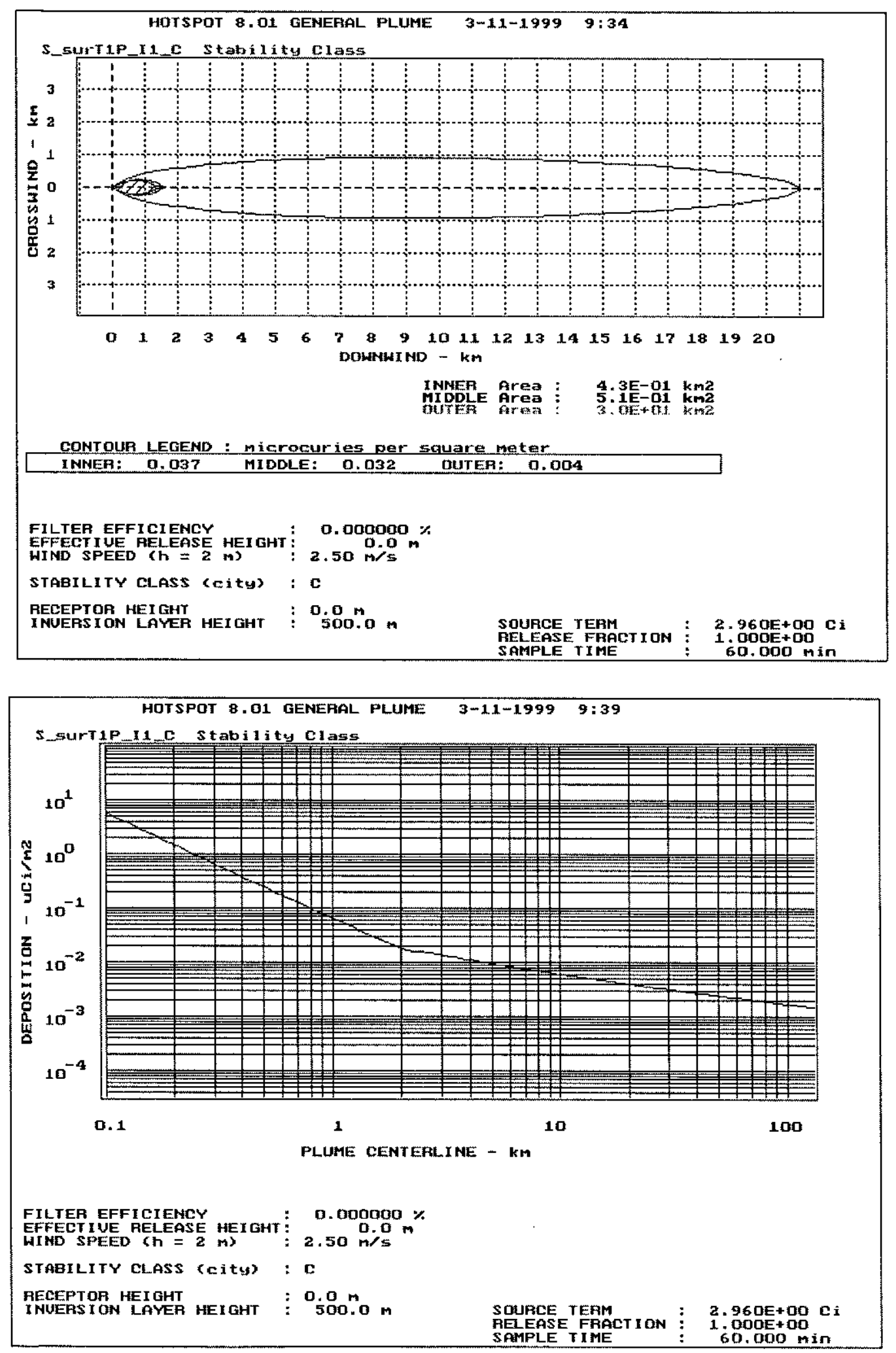

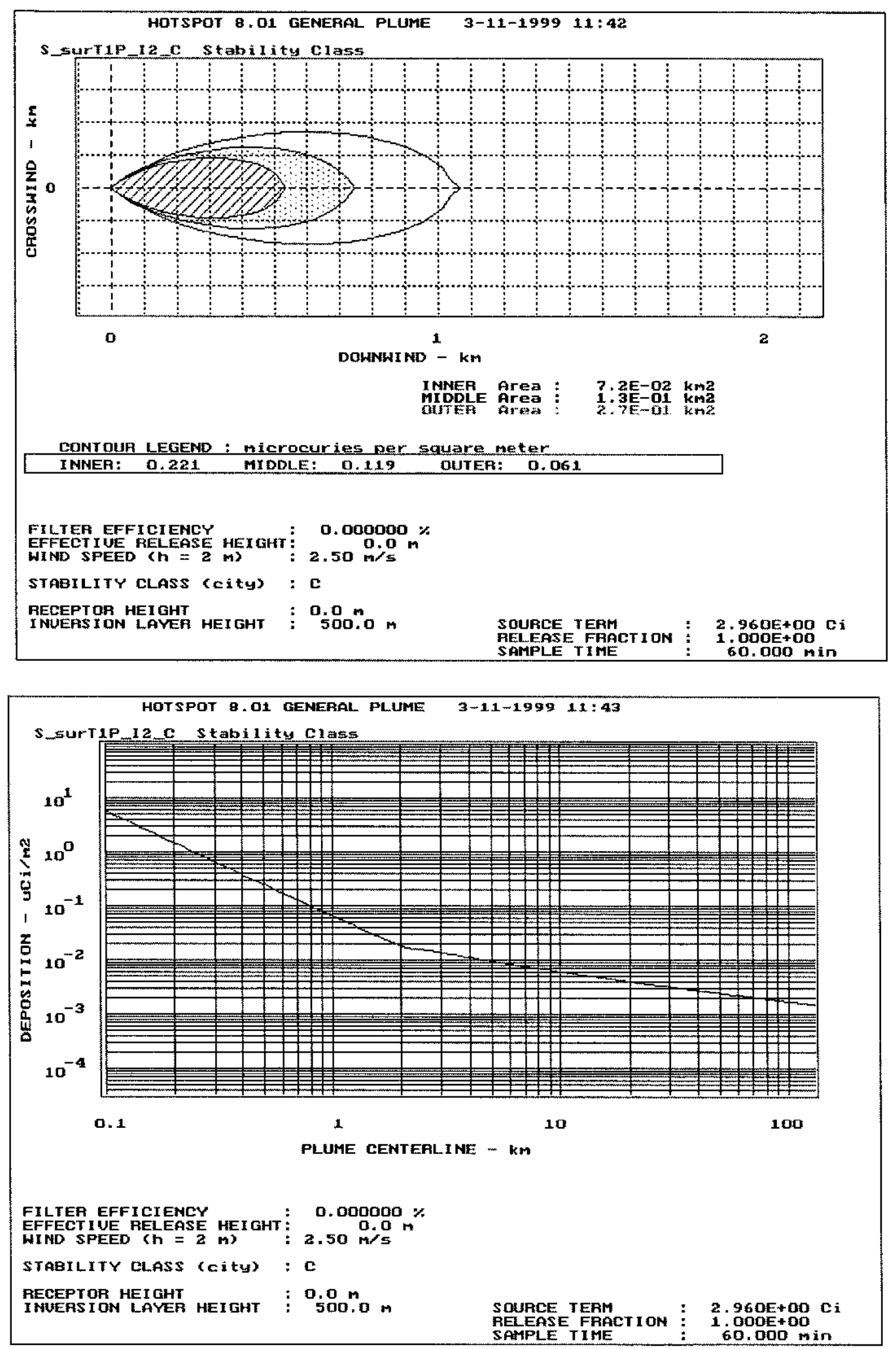

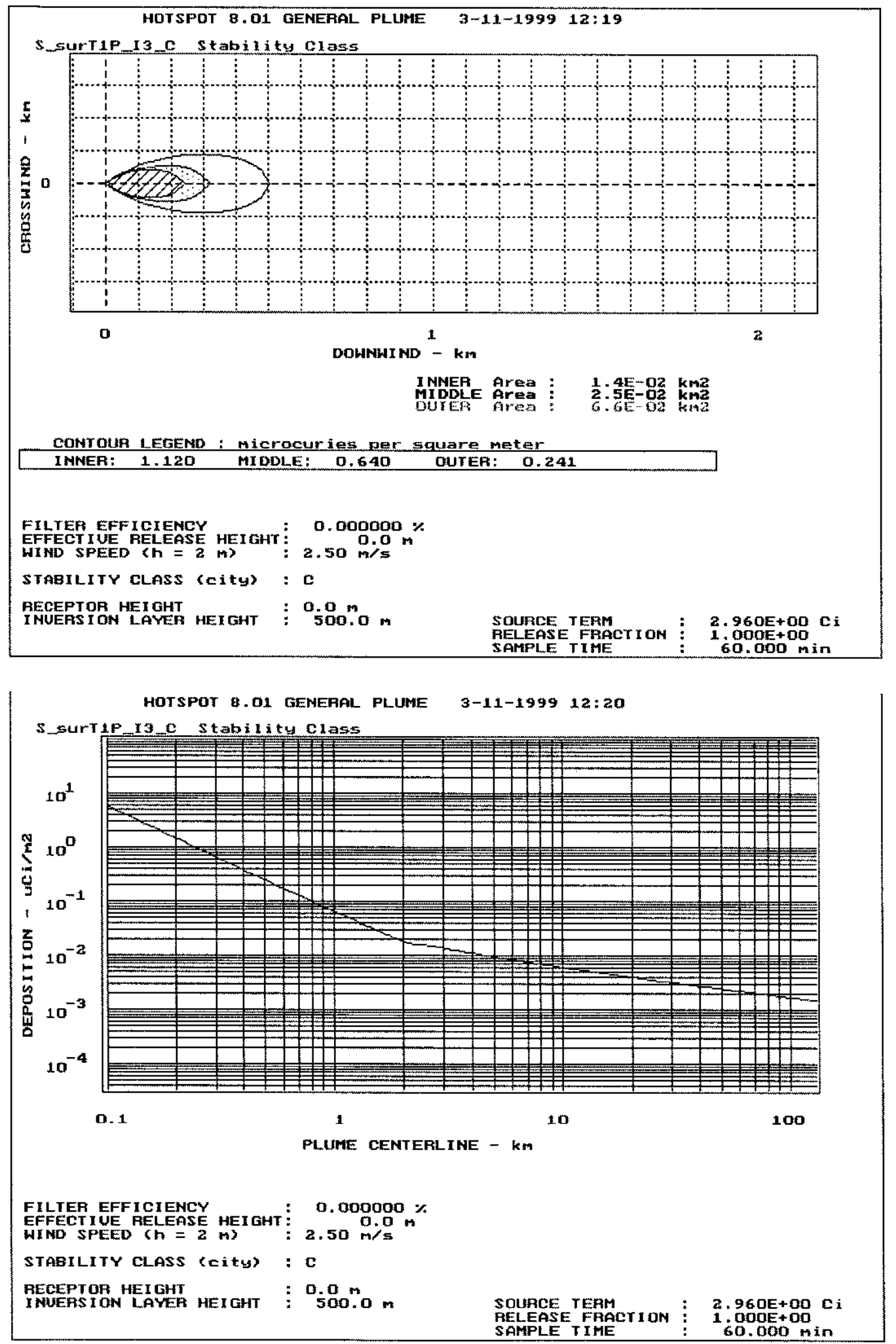

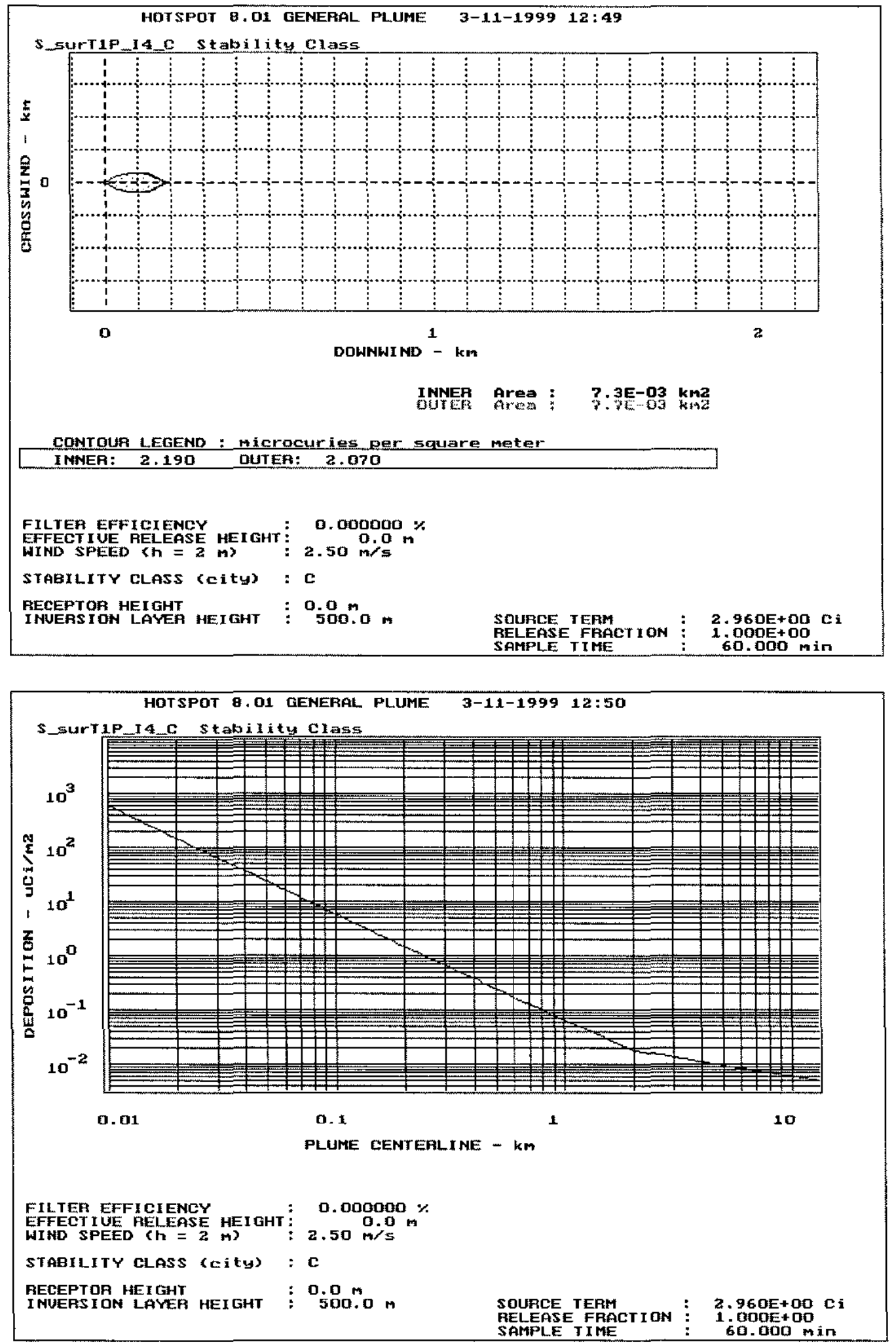


\section{Attachment T \\ HOTSPOT results - average meteorology, $10 \mathrm{~cm} \mathrm{~s}^{-1}$}



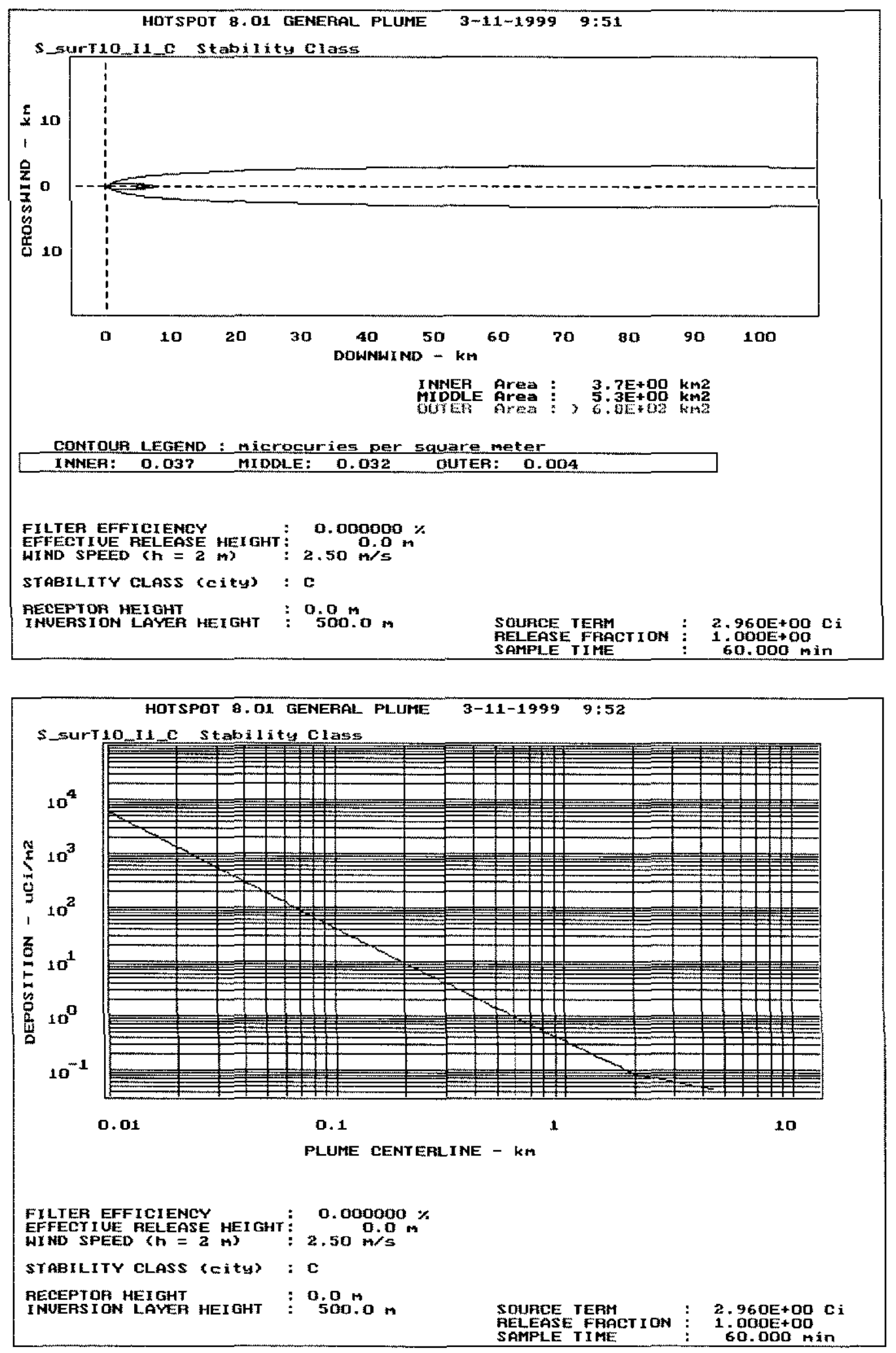

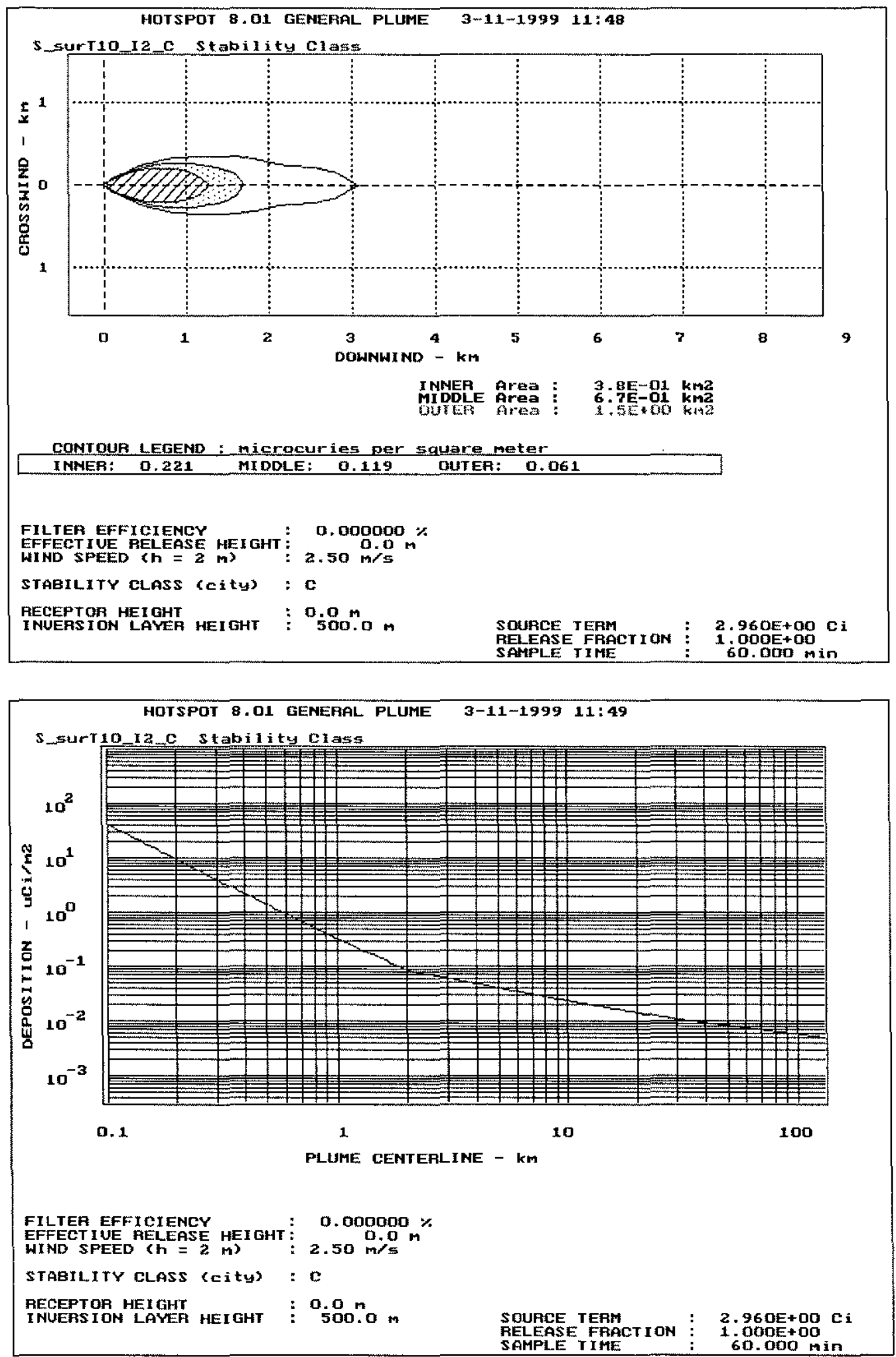

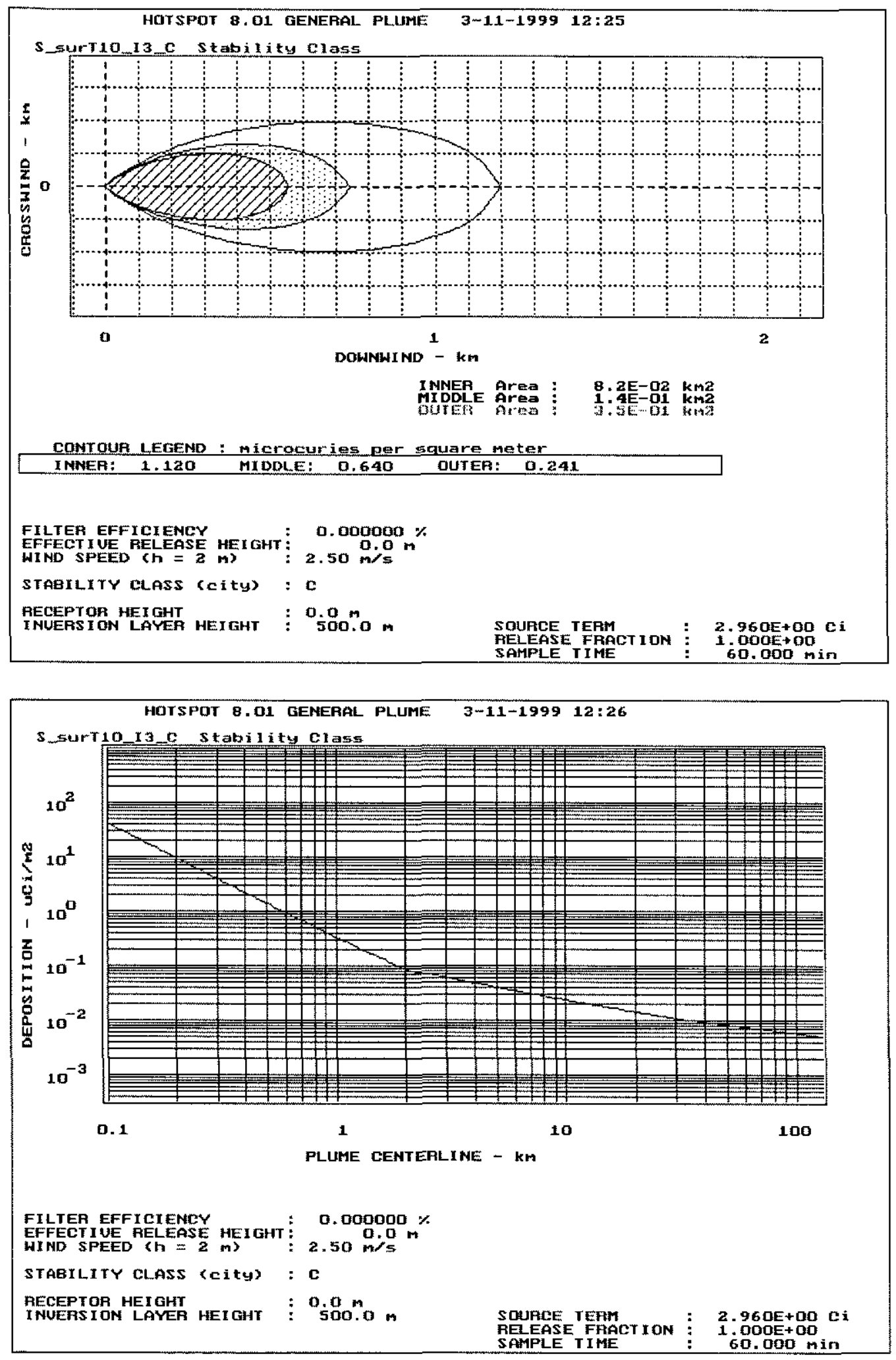

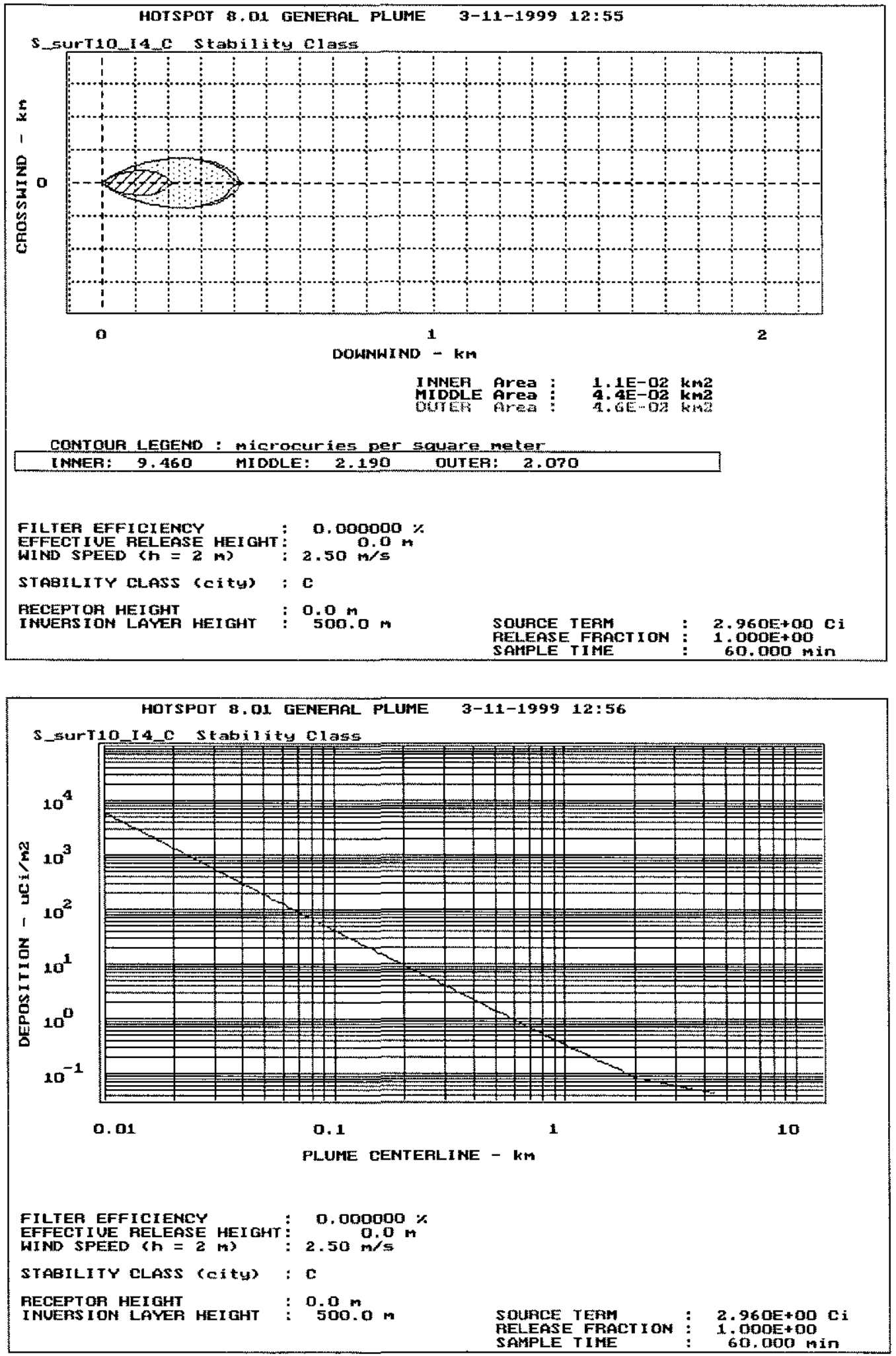

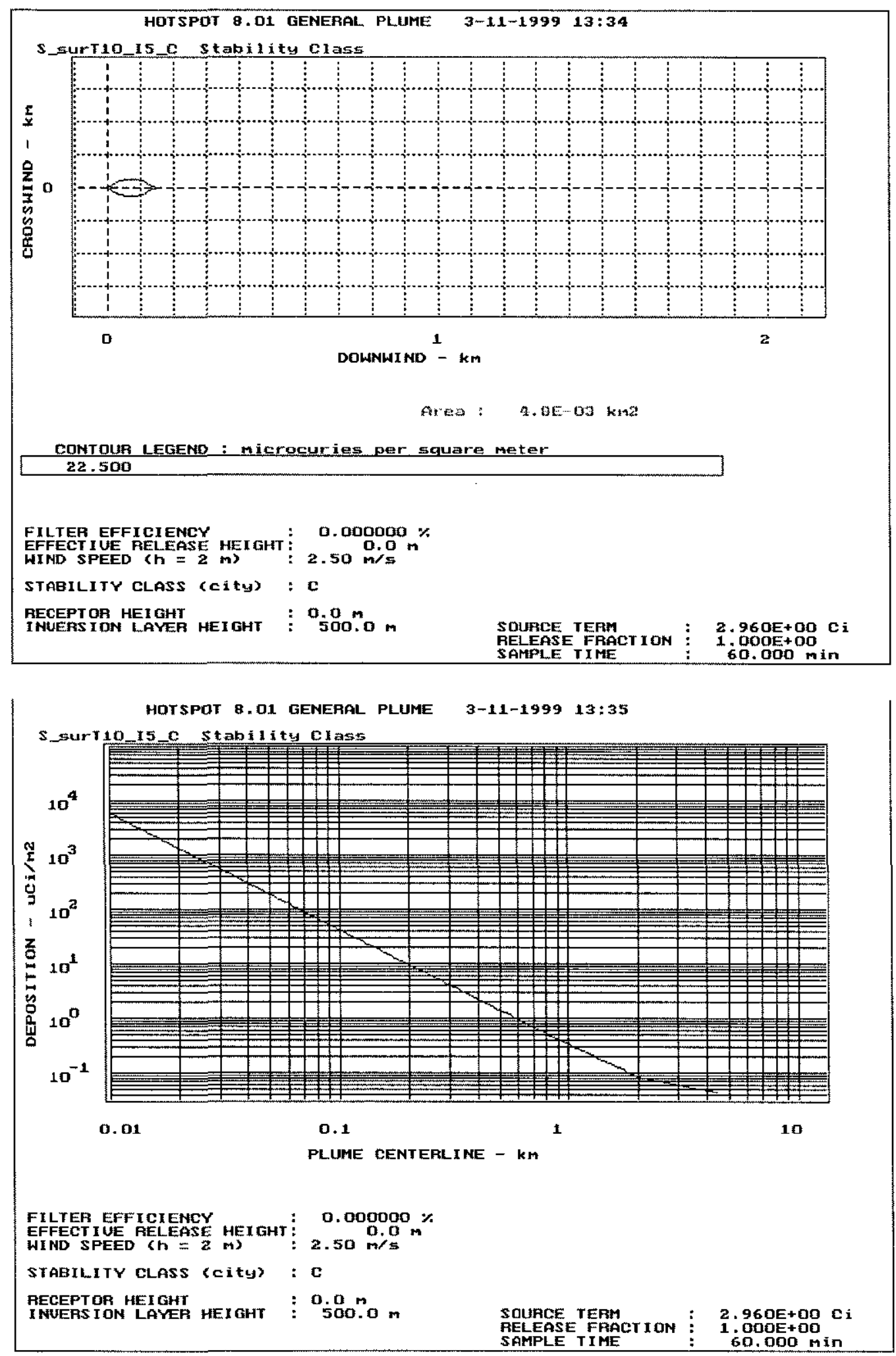


\section{Attachment U}

HOTSPOT results - adverse meteorology, $0.1 \mathrm{~cm} \mathrm{~s}^{-1}$ 

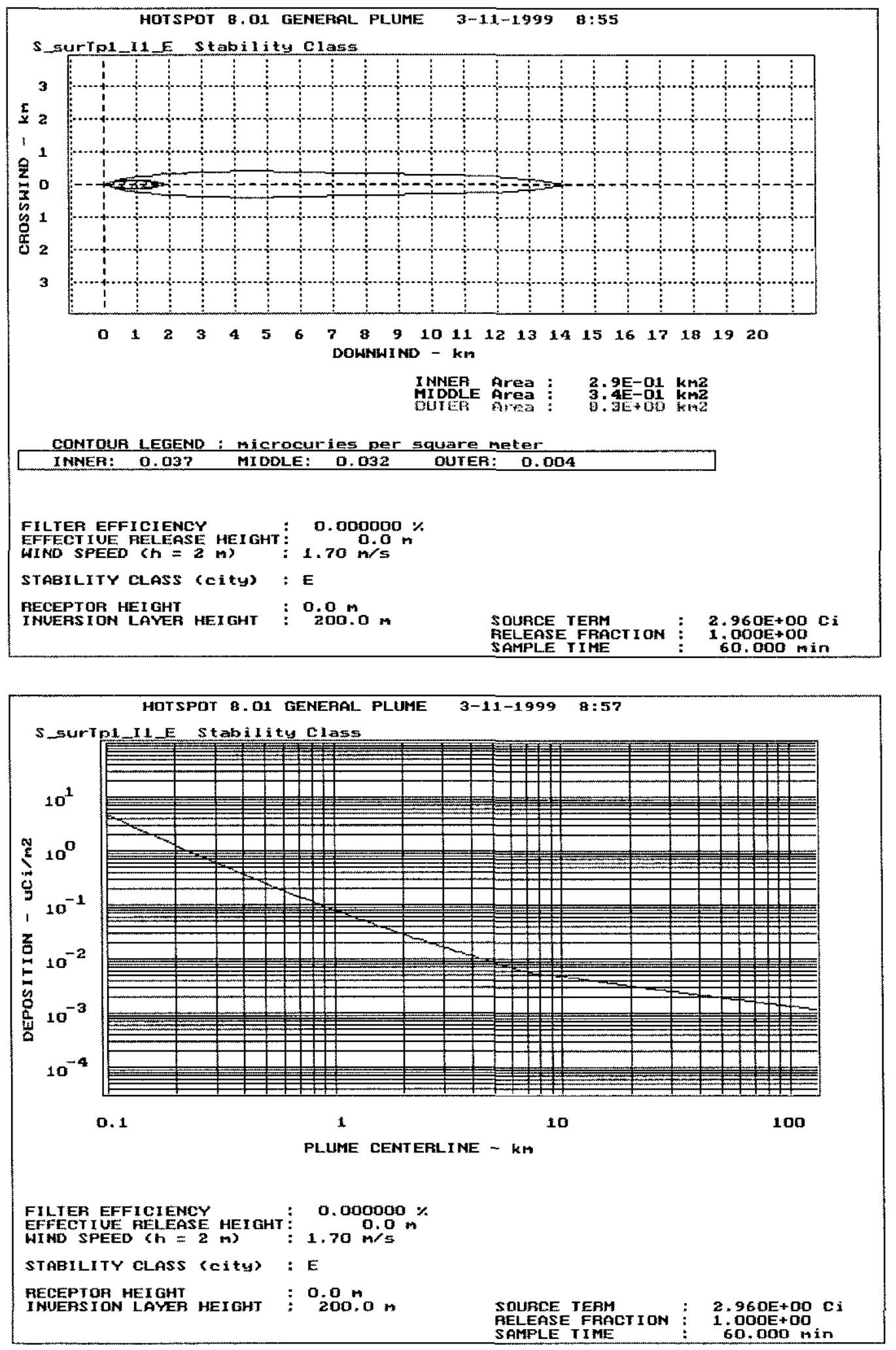

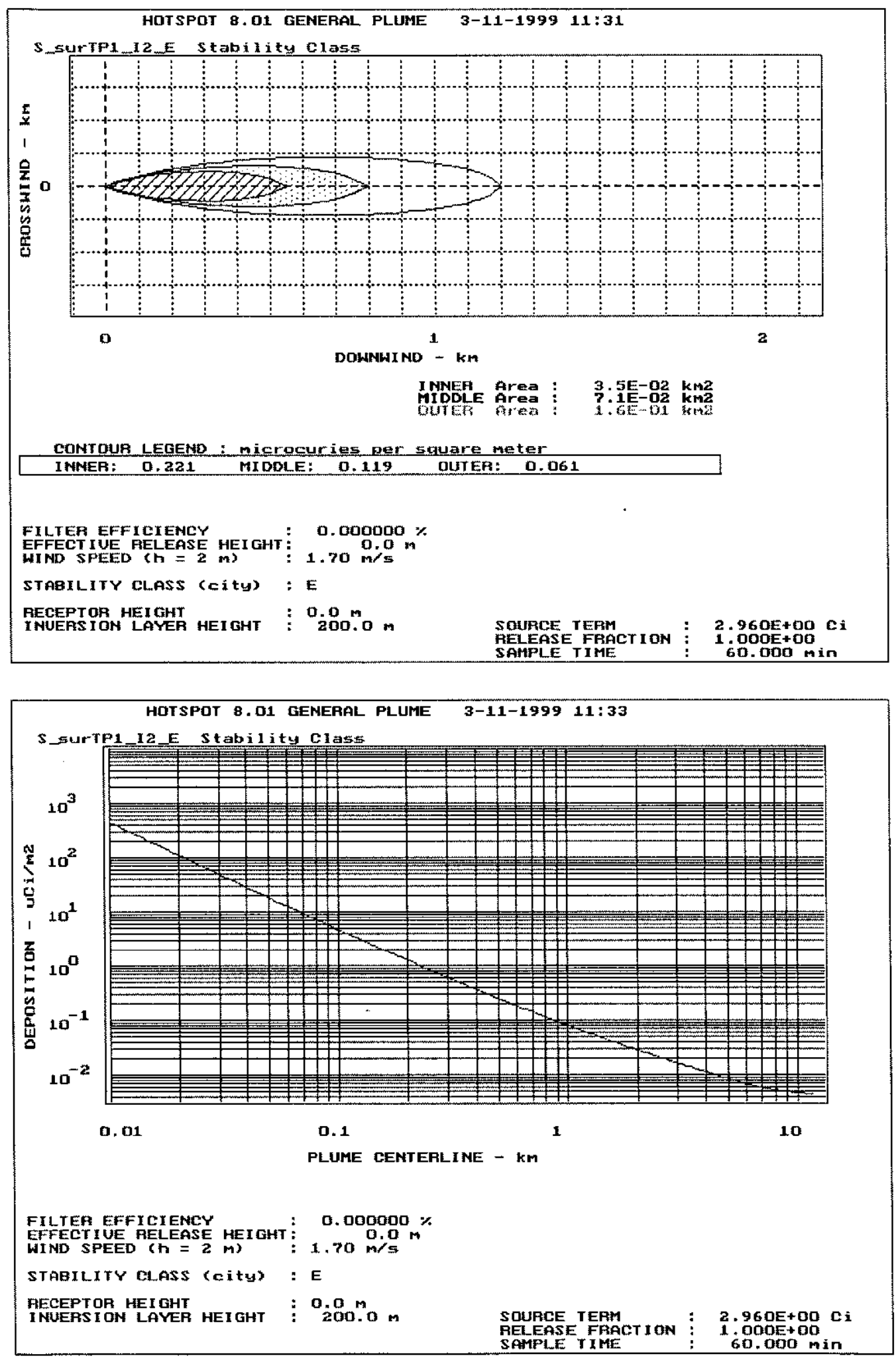

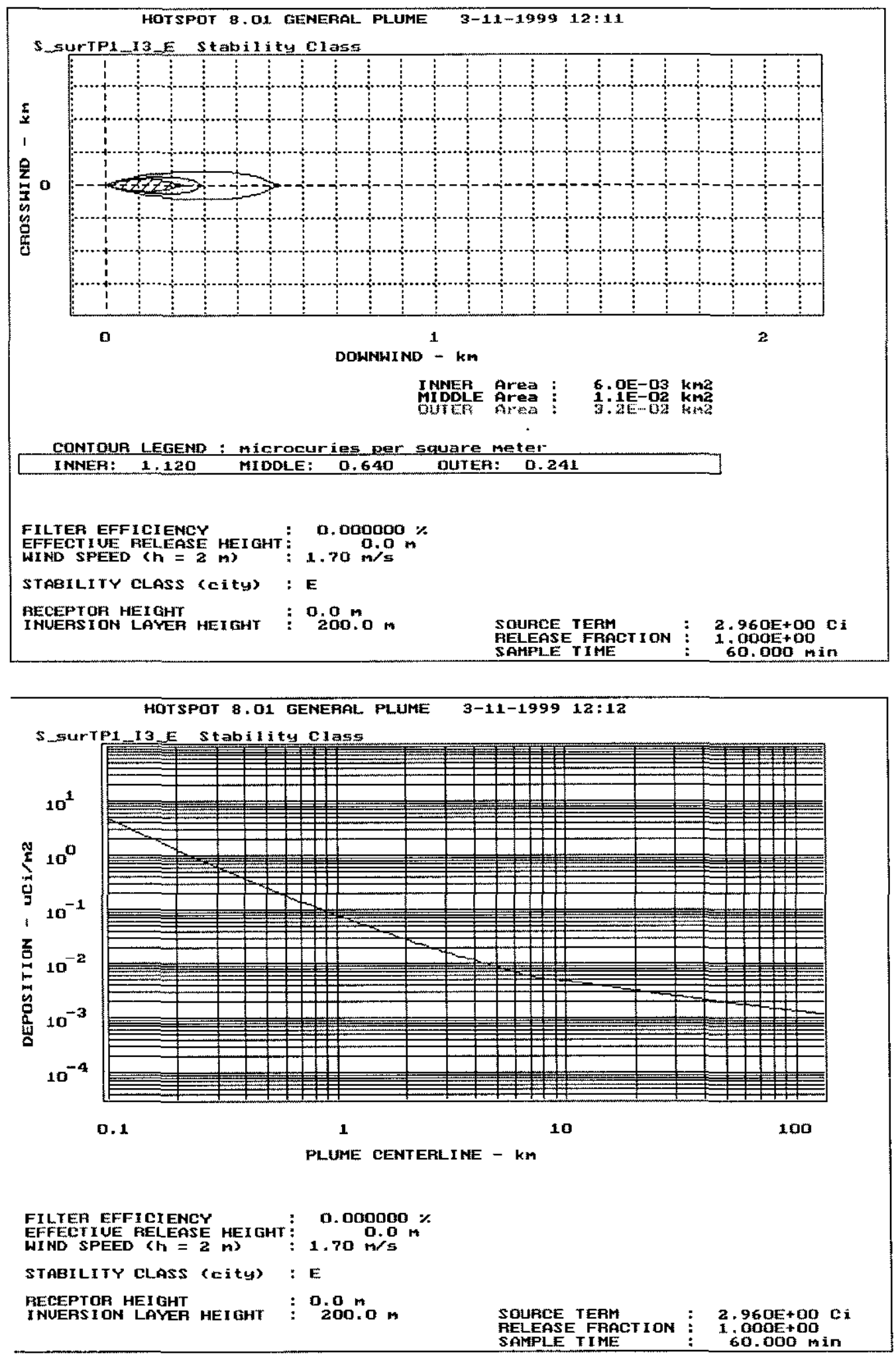

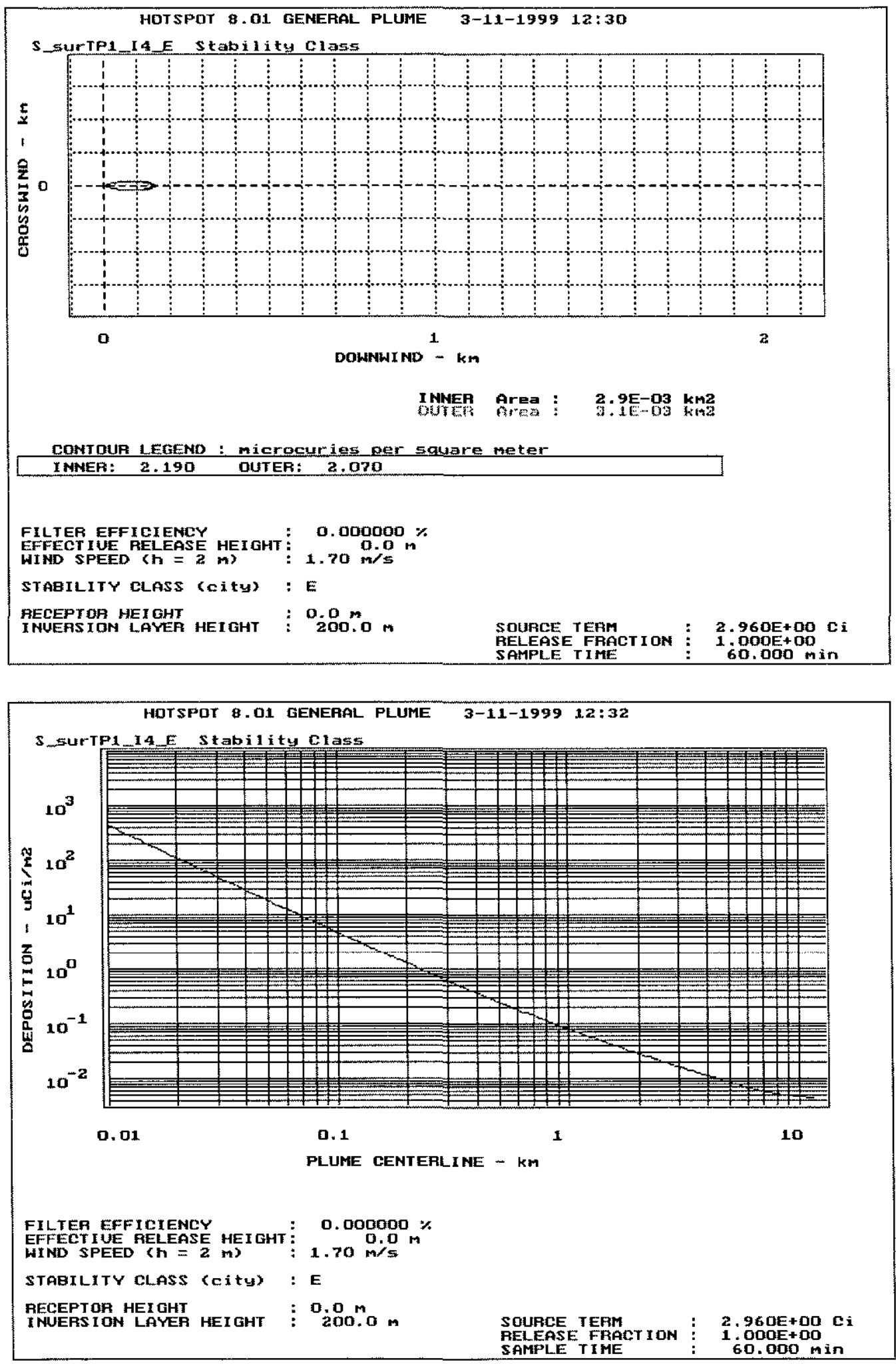


\section{Attachment V}

HOTSPOT results - adverse meteorology, $1.0 \mathrm{~cm} \mathrm{~s}^{-1}$ 

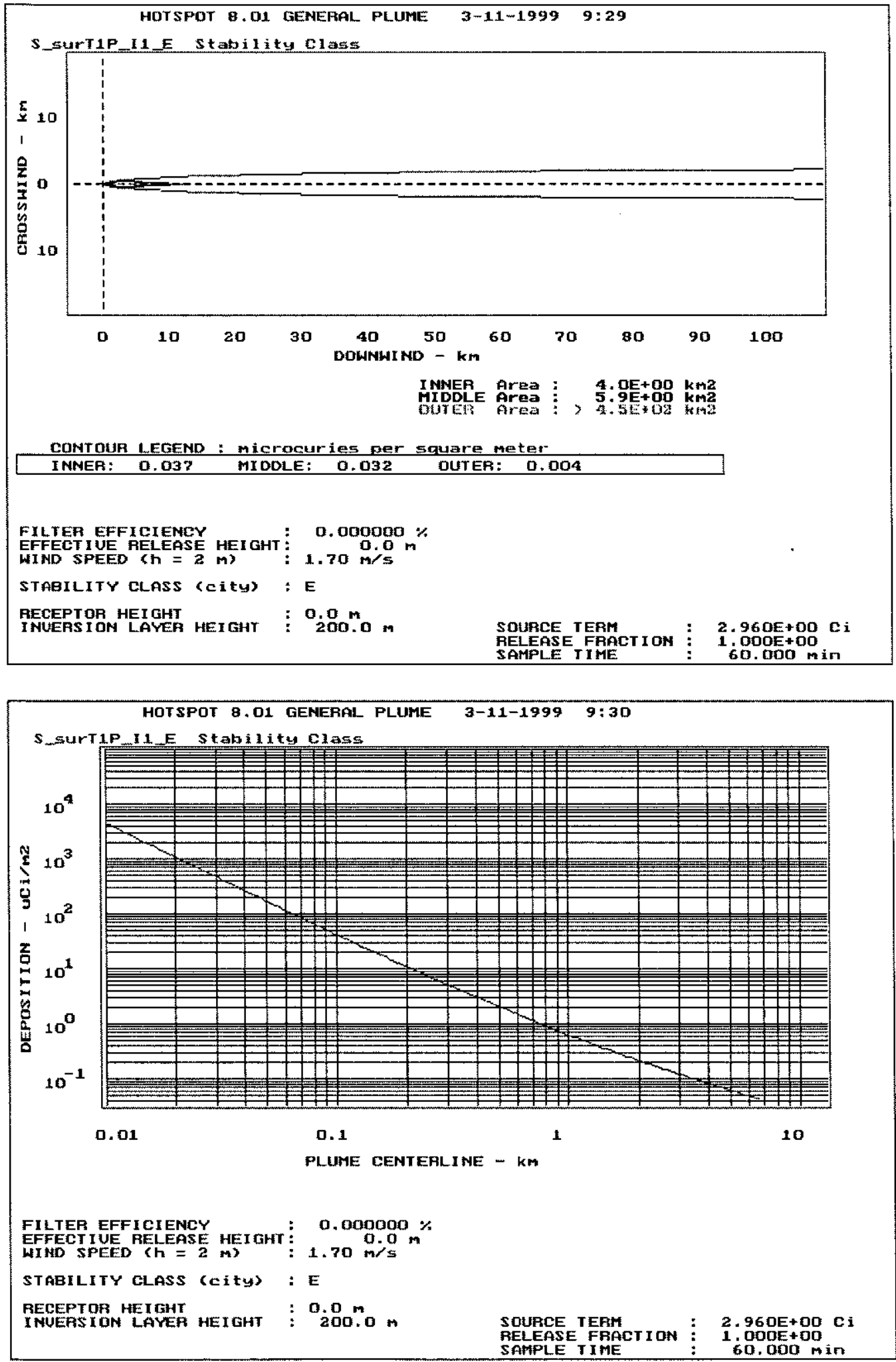

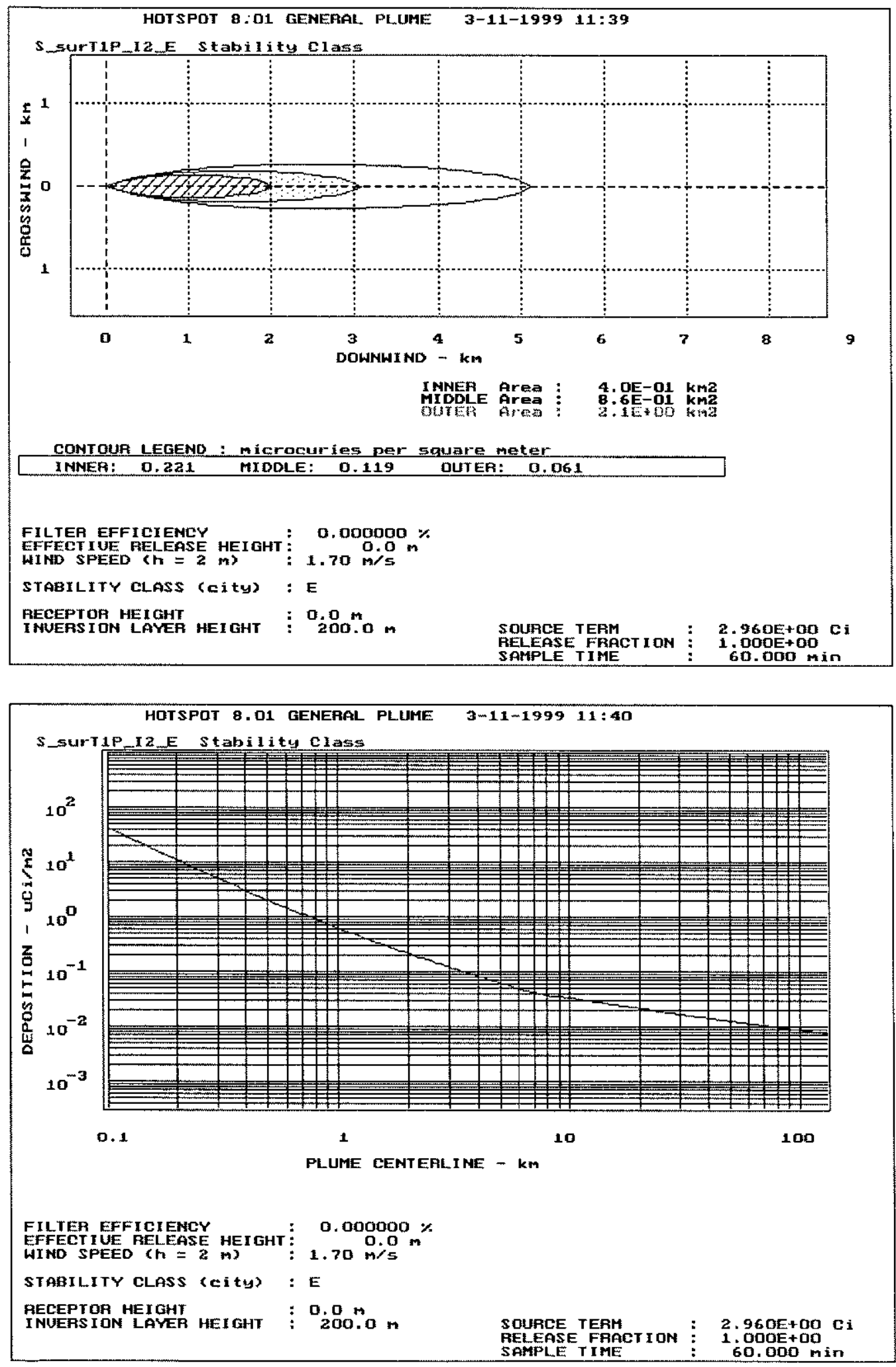

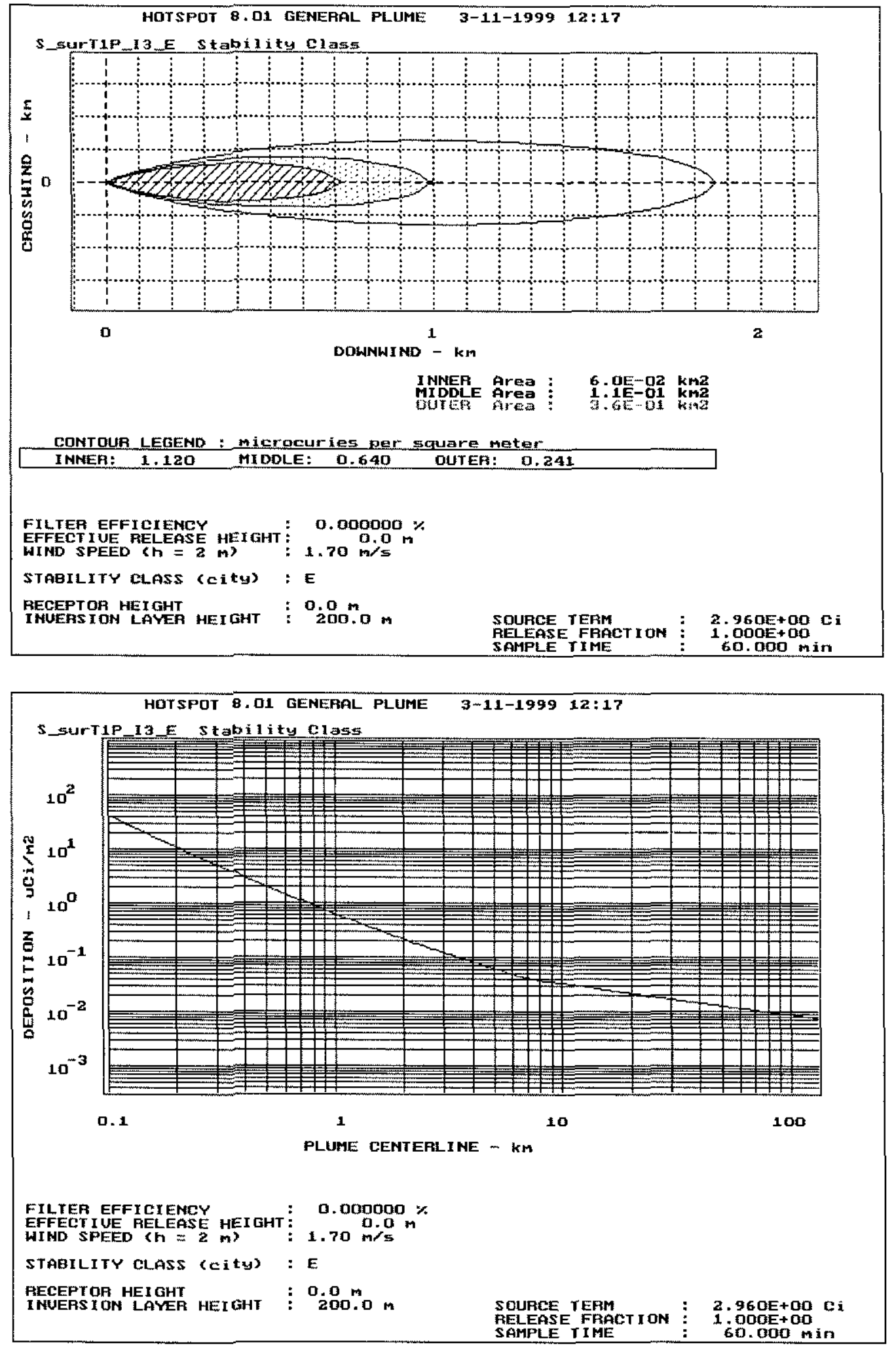

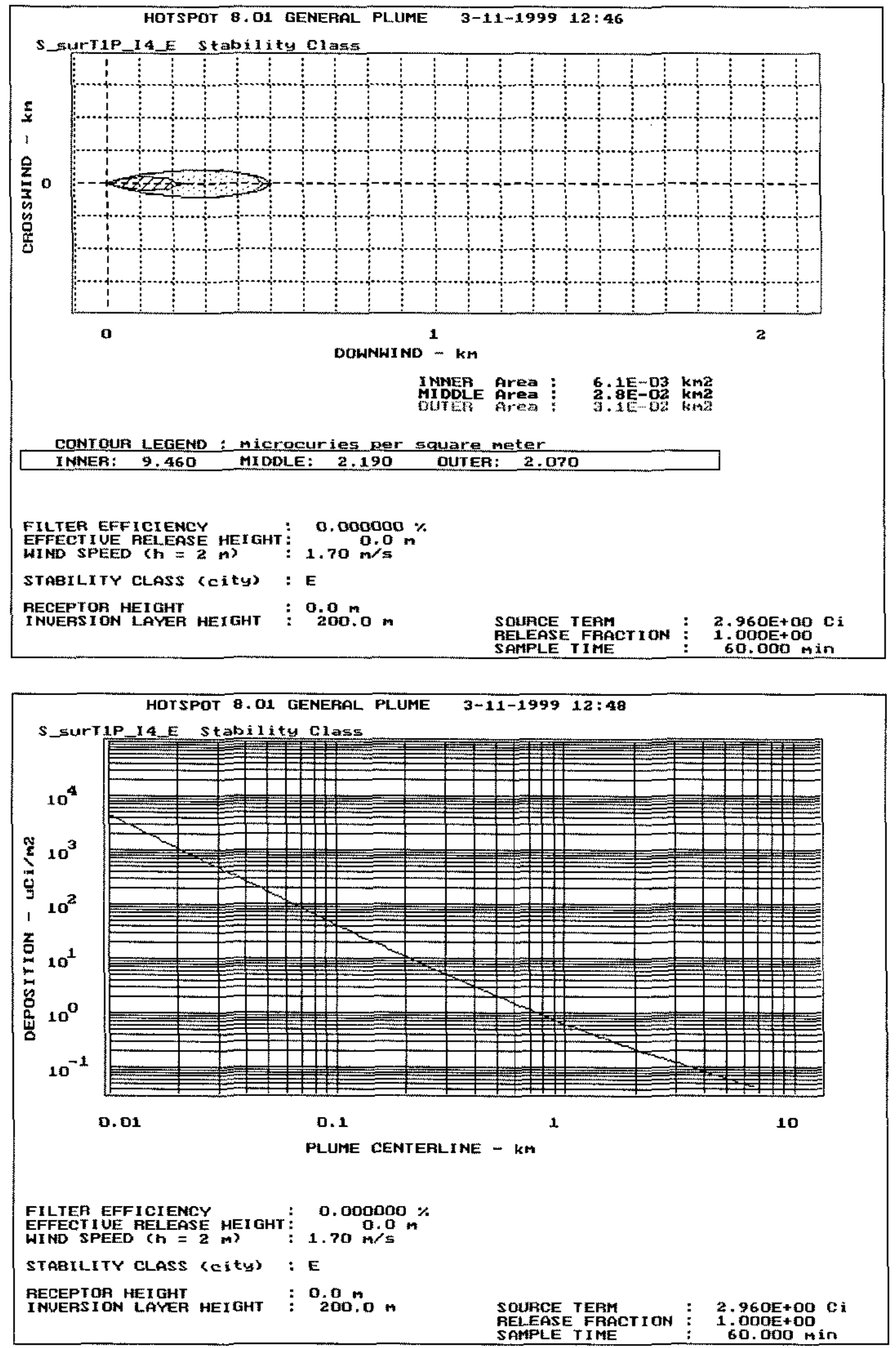

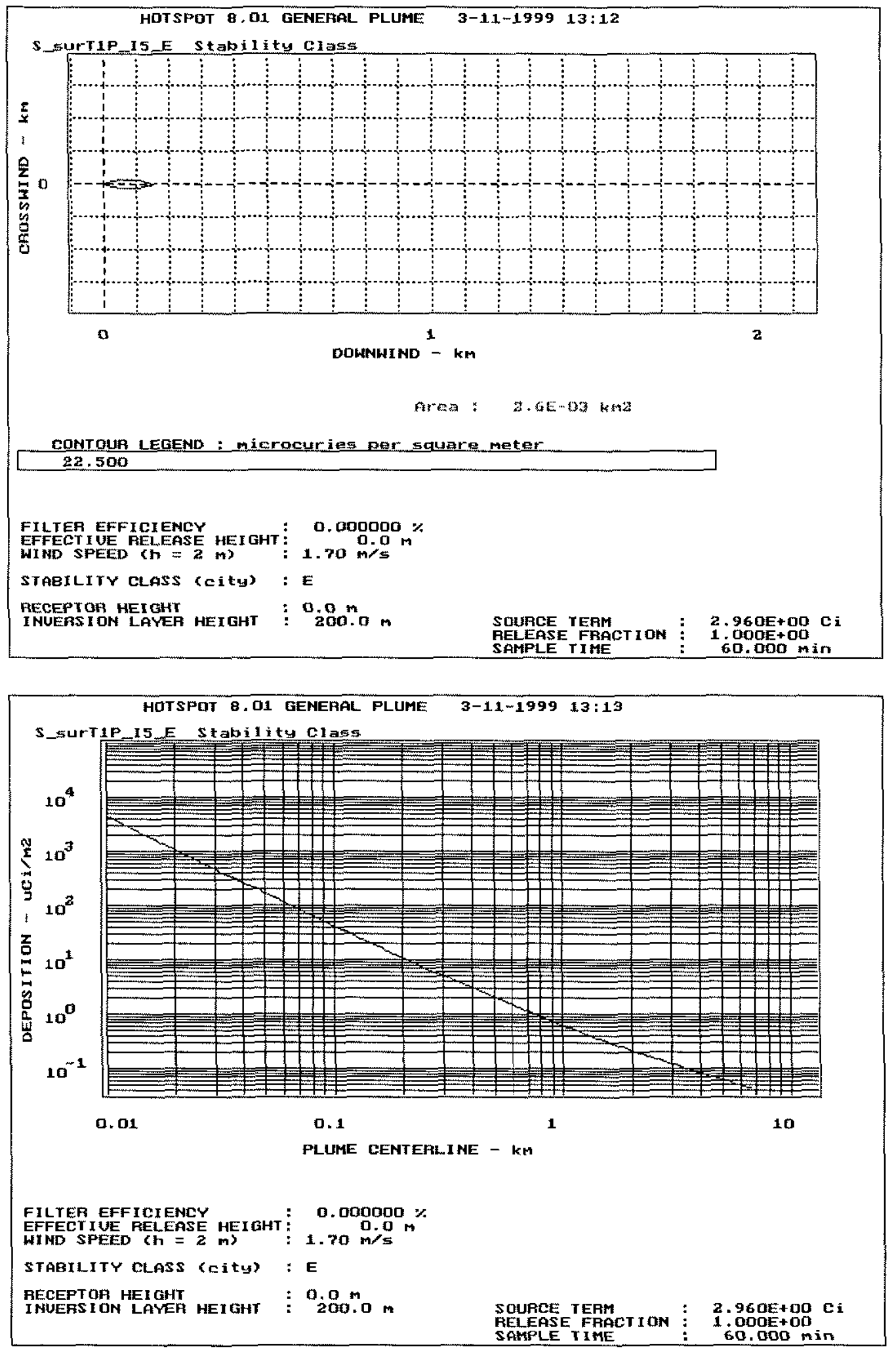


\section{Attachment $\mathbf{W}$}

HOTSPOT results - adverse meteorology, $10 \mathrm{~cm} \mathrm{~s}^{-1}$ 

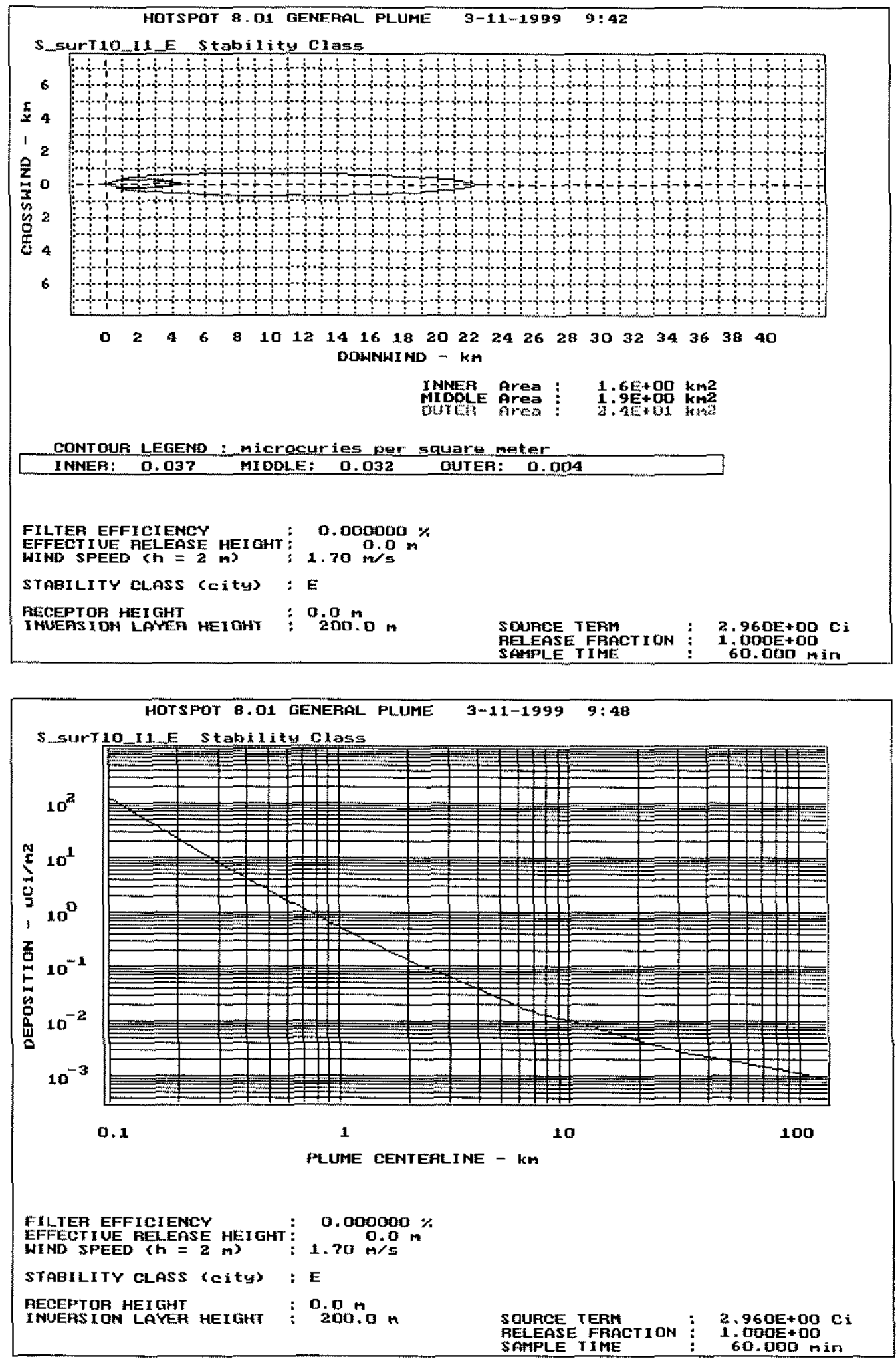

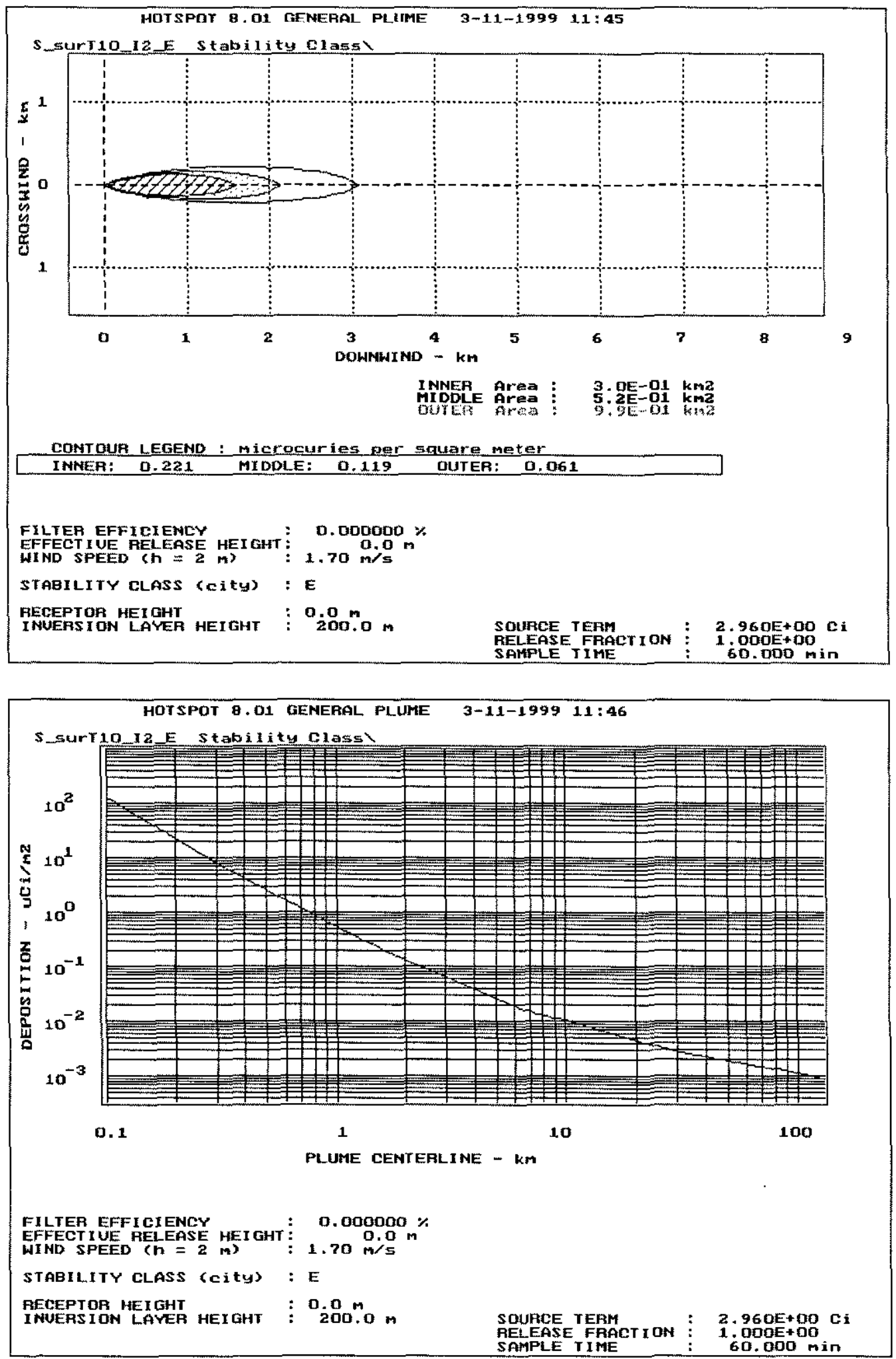

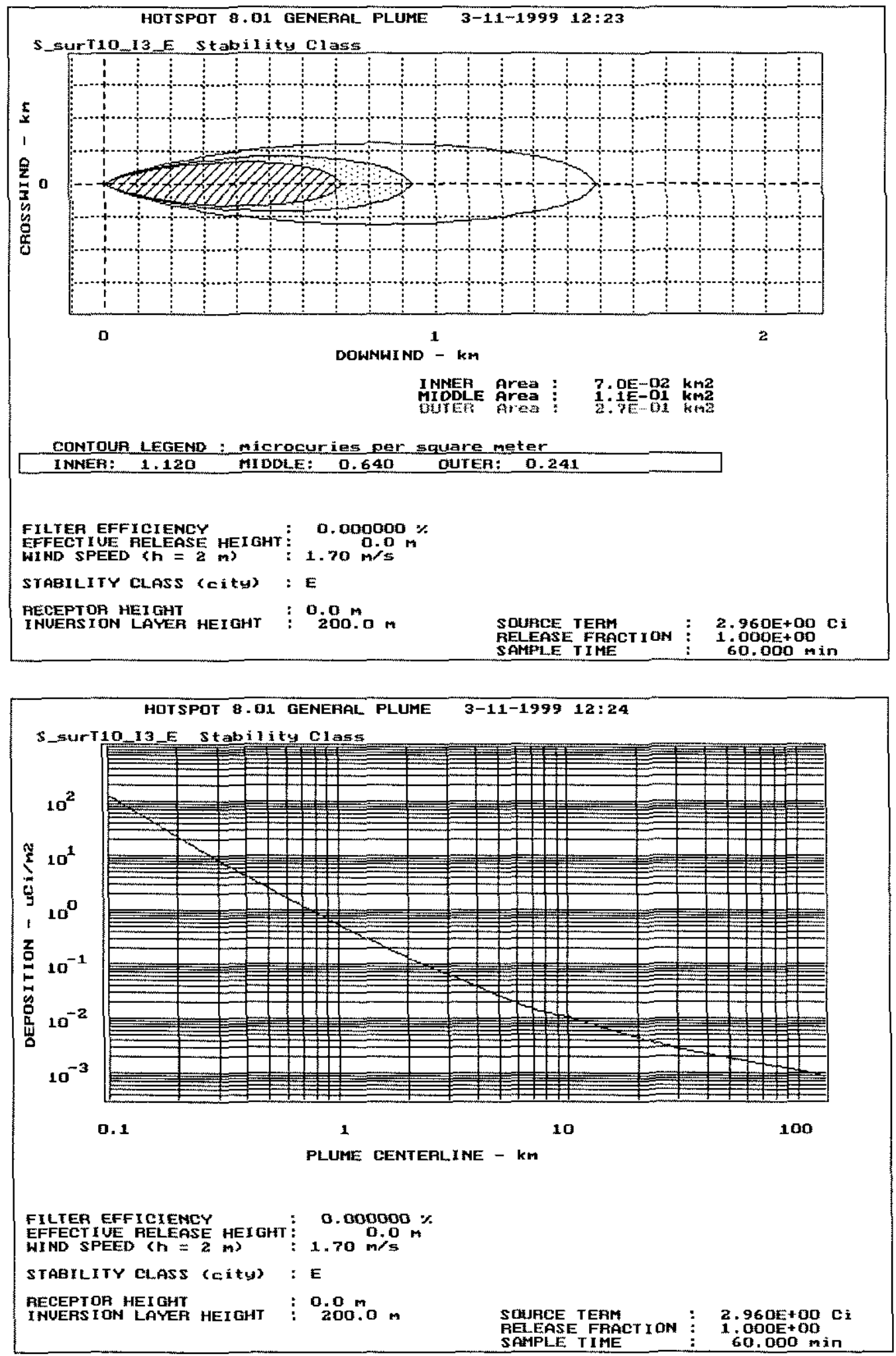

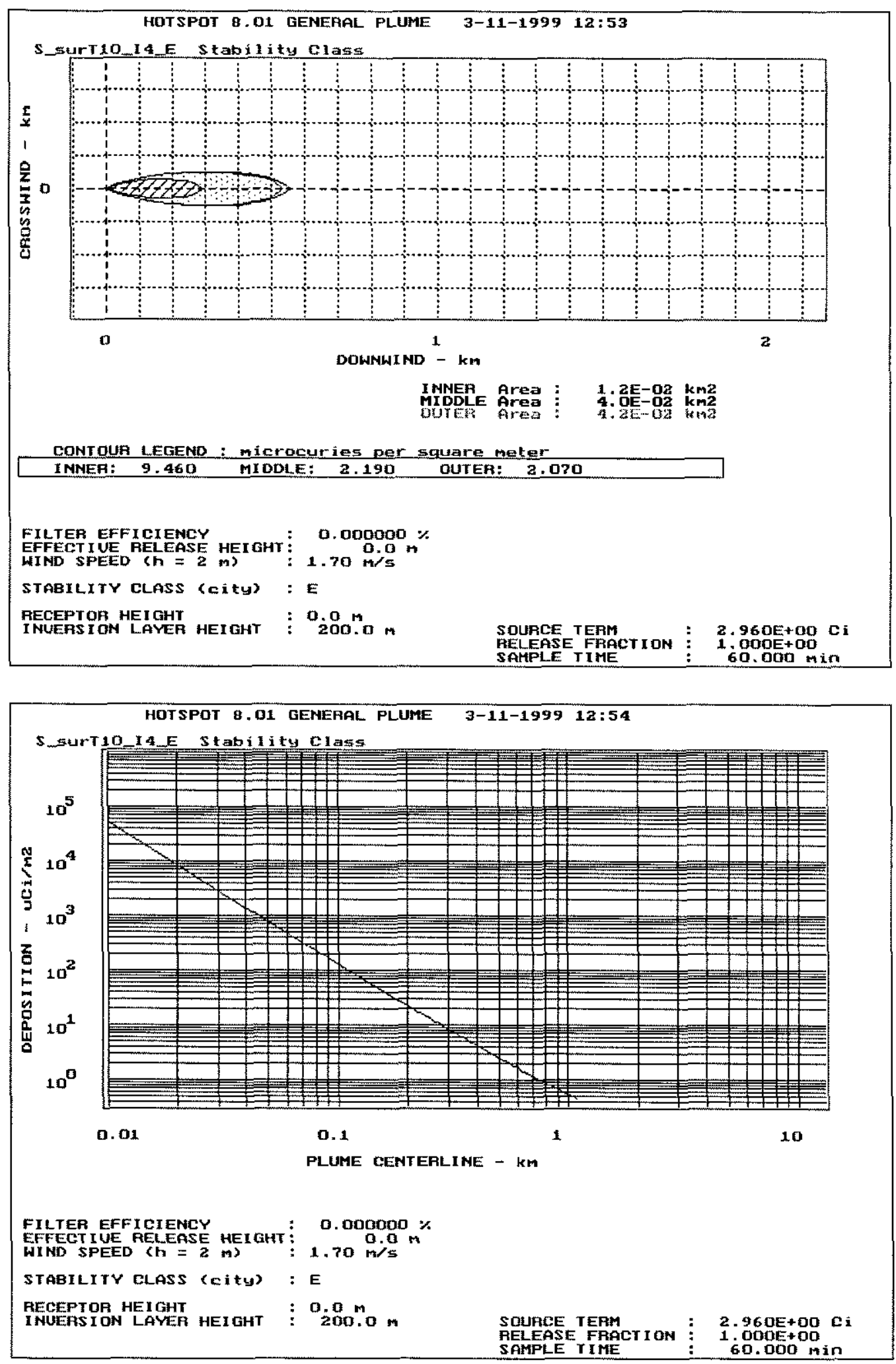

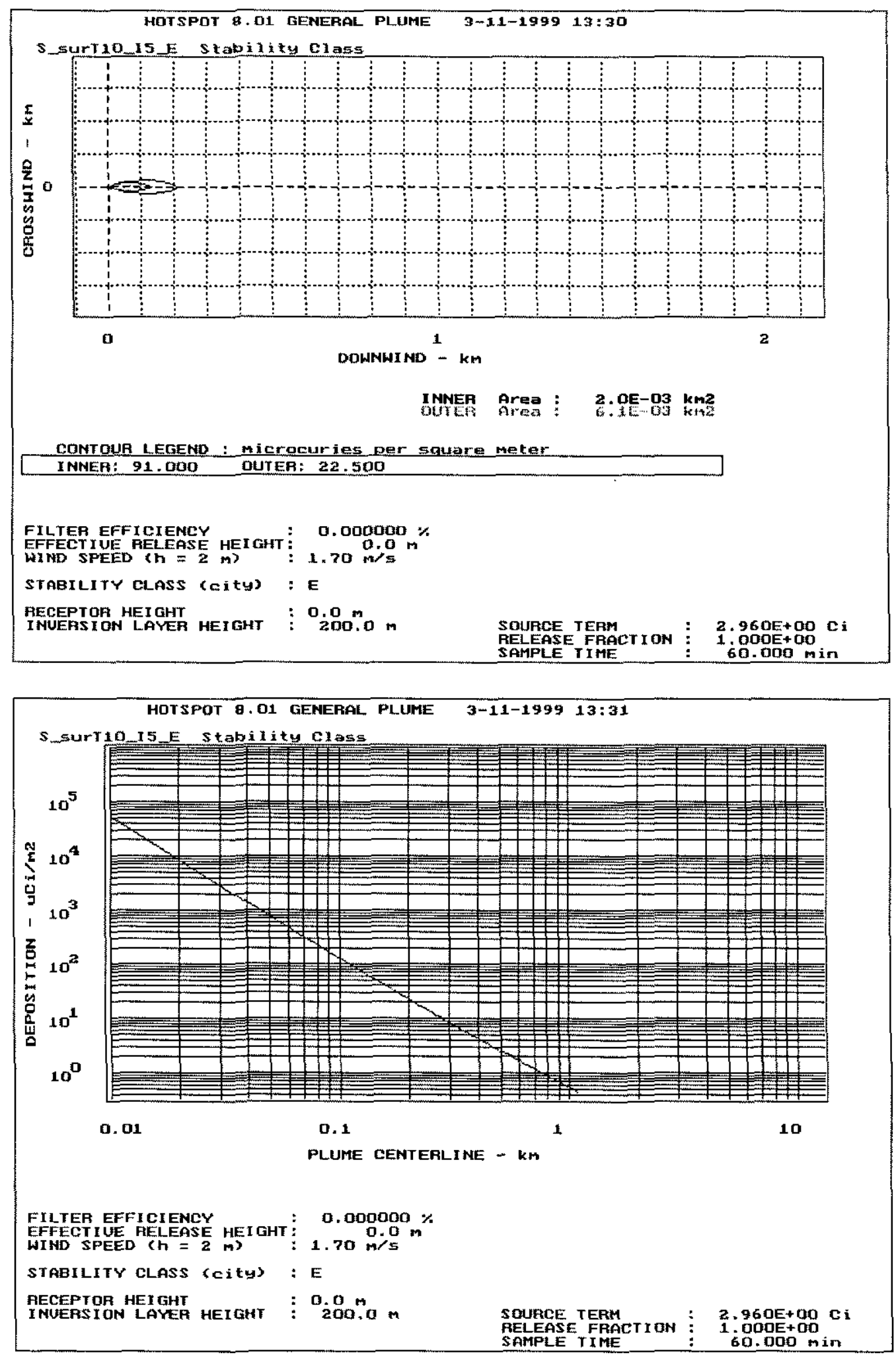